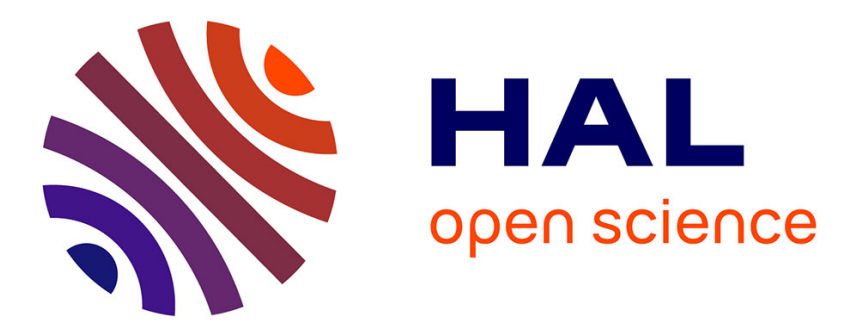

\title{
Automatic insertion of a turbulence model in the finite element discretization of the Navier-Stokes equations
}

Christine Bernardi, Tomas Chacon-Rebollo, Frédéric Hecht, Roger

Lewandowski

\section{- To cite this version:}

Christine Bernardi, Tomas Chacon-Rebollo, Frédéric Hecht, Roger Lewandowski. Automatic insertion of a turbulence model in the finite element discretization of the Navier-Stokes equations. Mathematical Models and Methods in Applied Sciences, 2009, 19 (7), pp.1139-1183. 10.1142/S0218202509003747. hal-00173706

\section{HAL Id: hal-00173706 \\ https://hal.science/hal-00173706}

Submitted on 20 Sep 2007

HAL is a multi-disciplinary open access archive for the deposit and dissemination of scientific research documents, whether they are published or not. The documents may come from teaching and research institutions in France or abroad, or from public or private research centers.
L'archive ouverte pluridisciplinaire HAL, est destinée au dépôt et à la diffusion de documents scientifiques de niveau recherche, publiés ou non, émanant des établissements d'enseignement et de recherche français ou étrangers, des laboratoires publics ou privés. 


\title{
Automatic insertion of a turbulence model in the finite element discretization of the Navier-Stokes equations
}

by Christine Bernardi ${ }^{1}$, Tomás Chacón Rebollo ${ }^{1,2}$, Frédéric Hecht ${ }^{1}$ and Roger Lewandowski ${ }^{3}$

\begin{abstract}
We consider the finite element discretization of the Navier-Stokes equations locally coupled with the equation for the turbulent kinetic energy through an eddy viscosity. We prove a posteriori error estimates which allow to automatically determine the zone where the turbulent kinetic energy must be inserted in the Navier-Stokes equations and also to perform mesh adaptivity in order to optimize the discretization of these equations. Numerical results confirm the interest of such an approach.
\end{abstract}

Résumé: Nous considérons une discrétisation par éléments finis des équations de NavierStokes couplées localement avec l'équation de l'énergie cinétique turbulente par une viscosité turbulente. Nous prouvons des estimations d'erreur a posteriori qui permettent de déterminer automatiquement la zône où l'énergie cinétique turbulente doit être insérée dans les équations de Navier-Stokes et simultanément d'adapter le maillage pour optimiser la discrétisation de ces équations. Des résultats numériques confirment l'intérêt d'une telle approche.

Key-words : Navier-Stokes Equations, Turbulence Models, Finite Elements Method, error estimates.

MCS Classification : 76F60, 65M60, 65M12.

1 Laboratoire Jacques-Louis Lions, C.N.R.S. \& Université Pierre et Marie Curie - Paris 6,

boîte 187, 4 place Jussieu, 75252 Paris Cedex 05, France.

bernardi@ann.jussieu.fr, hecht@ann.jussieu.fr

2 Departamento de Ecuaciones Diferenciales y Análisis Numerico, Universidad de Sevilla,

Tarfia s/n, 41012 Sevilla, Spain.

chacon@numer.us.es

3 IRMAR (U.M.R. 6625), Université de Rennes 1,

Campus de Beaulieu, 35042 Rennes Cedex 03, France.

roger.lewandowski@univ-rennes1.fr 



\section{Introduction.}

Let $\Omega$ be a connected bounded open set in $\mathbb{R}^{d}, d=2$ or 3 , with a Lipschitz-continuous boundary $\partial \Omega$. The following system models the stationary flow of a viscous incompressible turbulent fluid

$$
\begin{cases}-\operatorname{div}(\nu(k) \nabla \boldsymbol{u})+(\boldsymbol{u} \cdot \nabla) \boldsymbol{u}+\operatorname{grad} p=\boldsymbol{f} & \text { in } \Omega, \\ \operatorname{div} \boldsymbol{u}=0 & \text { in } \Omega, \\ -\operatorname{div}(\alpha(k) \nabla k)=\nu(k)|\nabla \boldsymbol{u}|^{2}-\frac{k \sqrt{k}}{\ell} & \text { in } \Omega, \\ \boldsymbol{u}=\boldsymbol{g} & \text { on } \partial \Omega, \\ k=0 & \text { on } \partial \Omega .\end{cases}
$$

The unknowns are the velocity $\boldsymbol{u}$, the pressure $p$ and the turbulent kinetic energy $k$, while the data are the distribution $\boldsymbol{f}$ and the function $\boldsymbol{g}$. The functions $\nu$ and $\alpha$ are positive and the parameter $\ell$, which represents the mixing length scale, is also positive. We refer to [35, Chap. 4] for the derivation of such a model. The main difficulty for its analysis is due to the fact that the right-hand member of the third equation is a priori not more than integrable. However the existence of a solution for similar problems when the function $\nu$ is bounded is proved in [19] and [29]. When the function $\nu$ is not bounded, the existence result is also established in [28, Chap. 5] for a scalar equation by a renormalization argument and in [27] for problem (1.1) by a regularity argument. It is also established in [3] and [4] in the case of two coupled fluids. Relying on the approach of [3] and [27], it can be checked that problem (1.1) admits a solution when the function $\nu$ satisfies some additional assumptions.

In this work, both for mathematical convenience and in order not to handle all difficulties together, we consider the simplified model

$$
\left\{\begin{array}{lc}
-\operatorname{div}(\nu(k) \nabla \boldsymbol{u})+(\boldsymbol{u} \cdot \nabla) \boldsymbol{u}+\operatorname{grad} p=\boldsymbol{f} & \text { in } \Omega, \\
\operatorname{div} \boldsymbol{u}=0 & \text { in } \Omega, \\
-\alpha \Delta k=\nu(k)|\nabla \boldsymbol{u}|^{2} & \text { in } \Omega, \\
\boldsymbol{u}=\mathbf{0} & \text { on } \partial \Omega \\
k=0 & \text { on } \partial \Omega
\end{array}\right.
$$

where $\alpha$ is now a positive constant. The replacement of nonhomogeneous boundary conditions by homogeneous ones, i.e., taking $\boldsymbol{g}$ equal to $\mathbf{0}$, is only aimed to avoid the technical difficulties of the Hopf lemma, see [20, Chap. IV, Lemma 2.3]. Note also [4, §2] that the replacement of $\operatorname{div}(\alpha(k) \nabla k)$ by $\alpha \Delta k$ comes from Kirchoff's change of unknown. Moreover, as suggested in [35, Chap. 4] and [10], we assume that the function $\nu$ admits the simple expansion

$$
\forall \xi \in \mathbb{R}_{+}, \quad \nu(\xi)=\nu_{0}+\nu_{1} \sqrt{\xi},
$$


for positive constants $\nu_{0}$ and $\nu_{1}$. Indeed, in practical situations, $\nu_{1}$ is a function which depends on the mixing length $\ell$ which appears in (1.1) and is approximatively of the same order; so, it is not restrictive to assume that it is constant in the case of problem (1.2).

Following the recent works [5] and [30], we are interested in the finite element discretization of the still rather complex system (1.2). Our approach relies on the following remark: Both experiments of measurements and numerical simulations indicate that, in a large part of the domain $\Omega$, the turbulent energy $k$ is negligible. So, our idea is to replace $\nu(k)$ by $\nu_{0}$ in all subdomains where the quantity $\nu_{1} \sqrt{k}$ can be neglected without increasing the global discretization error. Thus, we work with a finite element discretization of the reduced problem only. We refer to J. Hoffman and C. Johnson [24][25] for a similar approach but in the completely different framework of coupling the so-called Direct Numerical Simulation and Large Eddy Simulation algorithms.

Of course, the discretization of the reduced problem must be coupled with mesh adaptivity since small turbulence scales cannot be captured where the mesh is too coarse. The mathematical arguments for an optimal choice of the model coupled with mesh adaptivity relies on the a posteriori analysis of the discrete problem, and the main ideas for the automatic coupling of models are due to M. Braack and A. Ern [8]. Proving a posteriori estimates for nonlinear problems also relies on the approach of J. Pousin and J. Rappaz [38]. In addition, we need to handle some specific difficulties to build the error indicators related to the finite element discretization which are due to the nonlinear nature of the turbulent viscosity. We use arguments due to R. Verfürth [41, Chap. 3] for that. Note that, in any case, the deep links between the model and the mesh make the numerical analysis of the discrete problem more complex. However the numerical experiments that we present justify the interest of solving only a reduced problem.

An outline of the paper is as follows:

- The main ideas and the corresponding algorithm for the automatic insertion of the turbulence model are described in Section 2.

- In Section 3, we recall the arguments for proving the existence of a solution to problem (1.2).

- Section 4 is devoted to the analysis of a fixed reduced problem. A first a posteriori estimate is also derived.

- In Section 5, we describe the finite element discrete problem based on the variational formulation of the previous reduced problem. We prove the existence of a solution and a priori error estimates.

- In Section 6, we introduce two different families of error indicators, the first one being linked to the reduction of the problem and the second one to the finite element discretization. We prove a posteriori upper and lower bounds for the global error as a function of these indicators.

- In Section 7, we present some numerical experiments that are in good coherence with the analysis and justify the interest of automatic modeling.

- Some conclusions are given in Section 8. 


\section{The ideas for automatic modeling.}

The aim of automatic modeling in the present framework is to exhibit a partition of the domain $\Omega$ without overlap

$$
\bar{\Omega}=\bar{\Omega}_{t} \cup \bar{\Omega}_{\ell} \quad \text { and } \quad \Omega_{t} \cap \Omega_{\ell}=\emptyset,
$$

(where the indices $t$ and $\ell$ stand for turbulent and laminar, respectively), such that the finite element discrete problem associated with system (1.2) is solved with the viscosity $\nu(k)$ replaced by a constant $\nu_{0}$ on $\Omega_{\ell}$. This partition is considered as optimal when the domain $\Omega_{\ell}$ is chosen such that

- the total computation time is highly diminished,

- the error is not significantly increased,

in comparison with solving the discrete problem associated with the full system (1.2). Indeed, the turbulence zone is limited to a part of the domain in a large number of flows such as wakes, jets, boundary layers and so on.

From now on, system (1.2) with the viscosity $\nu(k)$ replaced by a constant $\nu_{0}$ on $\Omega_{\ell}$ is called "reduced problem", and its finite element discretization is called "reduced discrete problem".

We propose an iterative algorithm for the choice of $\Omega_{\ell}$ and $\Omega_{t}$ and of a corresponding triangulation, which is based on two families of error indicators (such indicators are defined in $(6.46)$ and $(6.4)-(6.5))$ : The first family deals with the modelization error and is made of error indicators $\eta_{K}^{m}$ for all elements $K$ of the triangulation which are contained in $\bar{\Omega}_{\ell}$ and the second one is made of the standard residual error indicators $\eta_{K}$ related to the finite element discretization for all elements $K$ of the triangulation. Note that an analogous strategy is used in [24] and [25] for the a posteriori error control of specific quantities.

Remark 2.1. In contrast with many other problems of automatic modeling, see [8], the choice of $\Omega_{t}$ and $\Omega_{\ell}$ cannot be made independently of the mesh adaptation. Indeed using the turbulence model in subdomains where the mesh is too coarse is meaningless since such a mesh cannot capture the small turbulence scales. So, in our case, the two types of adaptivity must be performed simultaneously.

InitializAtion steP: We first fix a triangulation $\mathcal{T}_{h}^{0}$ of the domain $\Omega$ such that the distance of the data $f$ to an appropriate finite element space relying on this mesh is smaller than a given tolerance $\eta^{*}$ (the importance of such a choice is brought to light in $[17])$. We take $\Omega_{t}^{0}=\emptyset$ and $\Omega_{\ell}^{0}=\Omega$. So we first solve the discrete Navier-Stokes equations (without eddy viscosity), next the discrete equation on $k$. Note indeed that the first and second line in (1.2) on one hand, and the third line on the other hand are uncoupled here and moreover that the third line is a linear problem. Thus we are in a position to compute the error indicators $\eta_{K}^{m}$ and $\eta_{K}$.

Adaptation step: We assume that a partition of $\Omega$ into two subdomains $\Omega_{t}^{n}$ and $\Omega_{\ell}^{n}$ satisfying (2.1) is known, together with a triangulation $\mathcal{T}_{h}^{n}$. We compute the solution of the associated reduced discrete problem, next the corresponding error indicators $\eta_{K}^{m}$ and $\eta_{K}$, together with the mean values $\bar{\eta}_{h}^{m}$ of the $\eta_{K}^{m}$ and the mean value $\bar{\eta}_{h}$ of the $\eta_{K}$. Next, we perform adaptivity in four substeps. 
1. Adaptivity due to modeling error.

All $K$ in $\mathcal{T}_{h}^{n}$ such that $\eta_{K}^{m}$ is $\geq \bar{\eta}_{h}^{m}$ (we recall that such $K$ are contained in $\Omega_{\ell}^{n}$ ) are inserted in a new domain $\widetilde{\Omega}_{t}^{n+1}$. More precisely, this new domain $\widetilde{\Omega}_{t}^{n+1}$ is the union of $\Omega_{t}^{n}$ and of these new $K$.

2. Decomposition regularization.

We perform the following regularization: Any element $K$ which is not imbedded in $\widetilde{\Omega}_{t}^{n+1}$ but is surrounded by elements which are imbedded in $\widetilde{\Omega}_{t}^{n+1}$, now belongs to the new domain $\Omega_{t}^{n+1}$. We skip the details for the construction of $\Omega_{t}^{n+1}$ and choose $\Omega_{\ell}^{n+1}$ such that (2.1) holds.

3. Mesh refinement due to the change of decomposition.

The triangulation is automatically refined in the elements which belong to $\Omega_{t}^{n+1}$ and not to $\Omega_{t}^{n}$, in order to capture the new scales of turbulence: Each of these elements is divided into smaller subelements according to the ratio $\bar{\eta}_{h}^{m} / \eta_{K}^{m}$. This gives rise to a new triangulation $\widetilde{\mathcal{T}}_{h}^{n+1}$.

4. Adaptivity due to discretization error.

When associating with each $K$ in $\widetilde{\mathcal{T}}_{h}^{n+1}$ which do not belong to $\mathcal{T}_{h}^{n}$ the $\eta_{K^{\prime}}$, where $K^{\prime}$ belongs to $\mathcal{T}_{h}^{n}$ and contains $K$, we are in a position to perform a standard finite element adaptivity strategy: The diameter of a new element contained in $K$ or containing $K$ is proportional to $h_{K}$ times the ratio $\bar{\eta}_{h} / \eta_{K}$, where $h_{K}$ denotes the diameter of $K$. We refer to [18] among others for more details on this procedure, specially in dimension $d=3$. This gives rise to the triangulation $\mathcal{T}_{h}^{n+1}$.

The adaptation step is of course iterated either a finite number of times or until both quantities

$$
\max _{K \in \mathcal{T}_{h}^{n}, K \subset \Omega_{\ell}^{n}} \eta_{K}^{m} \quad \text { and } \quad \max _{K \in \mathcal{T}_{h}^{n}} \eta_{K}
$$

become smaller than the tolerance $\eta^{*}$.

Remark 2.2. Observe that $\Omega_{t}^{n}$ is contained in $\Omega_{t}^{n+1}$ for all $n$. A more complex strategy must be used when working with the time-dependent system analogous to (1.2). Indeed, some triangles in $\Omega_{t}^{n}$ must go to $\Omega_{\ell}^{n+1}$ in order to handle vortices quickly moving from one part of the domain to another one. However we do not consider this situation here.

The numerical experiments which are presented in Section 7 justify the interest of this algorithm. Note also that the analysis which is performed in Sections 4 to 6 is perfectly adapted to it. In particular, it leads to the construction of appropriate error indicators $\eta_{K}^{m}$ and $\eta_{K}$ which make it very efficient. 


\section{About the continuous problem.}

Throughout this section, we make the following non restrictive assumptions on the function $\nu$ :

- It is a positive function on $\mathbb{R}$ satisfying

$$
\forall \xi \in \mathbb{R}, \quad \nu(\xi) \geq \nu_{0}
$$

for a positive constant $\nu_{0}$.

- It is bounded on $\mathbb{R}$, i.e.

$$
\forall \xi \in \mathbb{R}, \quad \nu(\xi) \leq \nu_{2}
$$

for a positive constant $\nu_{2}$.

- It is continuous on $\mathbb{R}$.

Note that assumption (3.2) can be avoided: Recent results prove that most of the properties below still hold in the two-dimensional scalar case [12] or when the function $\nu$ is concave [27], but without convection term. However we prefer to work with (3.2), since this does not induce any restriction of the model (in practical situations, $k$ is always bounded).

We use the standard notation for the Sobolev spaces $W^{m, p}(\Omega)$ and $W_{0}^{m, p}(\Omega)$ when $m$ is a positive integer and $1 \leq p \leq+\infty$. Moreover, with $1<p<+\infty$ and $\frac{1}{p}+\frac{1}{p^{\prime}}=1$, $W^{-m, p^{\prime}}(\Omega)$ is defined as the dual space of $W_{0}^{m, p}(\Omega)$ (see [1, Thm 3.8] for its characterization) and the corresponding duality pairing is denoted by $\langle\cdot, \cdot\rangle$. We also need the spaces $H^{s}(\Omega)$ for positive real numbers $s$. As usual, $L_{0}^{2}(\Omega)$ stands for the space of functions in $L^{2}(\Omega)$ with a null integral on $\Omega$.

From now on, we fix a real number $r>d$ and take $r^{\prime}$ such that

$$
\frac{1}{r}+\frac{1}{r^{\prime}}=1
$$

Thus, we consider the variational problem,

Find $(\boldsymbol{u}, p, k)$ in $H_{0}^{1}(\Omega)^{d} \times L_{0}^{2}(\Omega) \times W_{0}^{1, r^{\prime}}(\Omega)$ such that

$$
\begin{aligned}
& \forall \boldsymbol{v} \in H_{0}^{1}(\Omega)^{d}, \quad \int_{\Omega} \nu(k) \nabla \boldsymbol{u}: \nabla \boldsymbol{v} d \boldsymbol{x}+\int_{\Omega}(\boldsymbol{u} \cdot \nabla) \boldsymbol{u} \cdot \boldsymbol{v}(\boldsymbol{x}) d \boldsymbol{x} \\
& -\int_{\Omega}(\operatorname{div} \boldsymbol{v})(\boldsymbol{x}) p(\boldsymbol{x}) d \boldsymbol{x}=\langle\boldsymbol{f}, \boldsymbol{v}\rangle, \\
& \forall q \in L_{0}^{2}(\Omega), \quad-\int_{\Omega}(\operatorname{div} \boldsymbol{u})(\boldsymbol{x}) q(\boldsymbol{x}) d \boldsymbol{x}=0, \\
& \forall \chi \in W_{0}^{1, r}(\Omega), \quad \alpha \int_{\Omega} \operatorname{grad} k \cdot \operatorname{grad} \chi d \boldsymbol{x}=\int_{\Omega} \nu(k)|\nabla \boldsymbol{u}|^{2} \chi(\boldsymbol{x}) d \boldsymbol{x} .
\end{aligned}
$$

Indeed standard arguments yield that this formulation is fully equivalent to problem (1.2). Moreover the product $\nu(k)|\nabla \boldsymbol{u}|^{2}$ belongs to $L^{1}(\Omega)$ and it follows from the Sobolev imbedding theorem that $\chi$ belongs to $L^{\infty}(\Omega)$, so that the right-hand member of the third equation is well defined. 
We first state an a priori estimate on the solutions of problem (3.3). Note that the estimate concerning $\boldsymbol{u}$ is obviously derived by taking $\boldsymbol{v}$ equal to $\boldsymbol{u}$ in the first line of (3.3). The estimate concerning $p$ involves the standard inf-sup condition on the bilinear form: $(\boldsymbol{v}, q) \mapsto-\int_{\Omega}(\operatorname{div} \boldsymbol{v})(\boldsymbol{x}) q(\boldsymbol{x}) d \boldsymbol{x}$, see [20, Chap. I, Cor. 2.1]. We refer to [7] for the argument leading to the estimate concerning $k$.

Proposition 3.1. For any data $\boldsymbol{f}$ in $H^{-1}(\Omega)^{d}$, any solution $(\boldsymbol{u}, p, k)$ of problem (3.3) satisfies the following bound

$$
\begin{gathered}
\nu_{0}\|\boldsymbol{u}\|_{H^{1}(\Omega)^{d}} \leq c\|\boldsymbol{f}\|_{H^{-1}(\Omega)^{d}}, \\
\|p\|_{L^{2}(\Omega)} \leq c\left(\left(1+\frac{\nu_{2}}{\nu_{0}}\right)\|\boldsymbol{f}\|_{H^{-1}(\Omega)^{d}}+\frac{1}{\nu_{0}^{2}}\|\boldsymbol{f}\|_{H^{-1}(\Omega)^{d}}^{2}\right), \\
\alpha\|k\|_{W^{1, r^{\prime}(\Omega)}} \leq c \frac{\nu_{2}}{\nu_{0}^{2}}\|\boldsymbol{f}\|_{H^{-1}(\Omega)^{d}}^{2},
\end{gathered}
$$

for a constant $c$ only depending on $\Omega$ and $r$.

Proving the existence of a solution for problem (3.3) relies on a fixed point theorem. We refer to [29] for the details (see also [3] and [4] for analogous results in a more complex situation). As already hinted, the next theorem requires assumptions (3.1) and (3.2) (see [27] for other cases) and also the continuity of the function $\nu$.

Theorem 3.2. For any data $\boldsymbol{f}$ in $H^{-1}(\Omega)^{d}$, problem (3.3) admits a solution $(\boldsymbol{u}, p, k)$ in $H_{0}^{1}(\Omega)^{d} \times L_{0}^{2}(\Omega) \times W_{0}^{1, r^{\prime}}(\Omega)$. Moreover this solution satisfies $(3.4)$.

Theorem 3.2 provides the existence result for problem (3.3) in the case we are interested in, i.e. when the viscosity $\nu$ is given by

$$
\forall \xi \in \mathbb{R}, \quad \nu(\xi)=\min \left\{\nu_{0}+\nu_{1} \sqrt{\xi_{+}}, \nu_{2}\right\},
$$

where $\xi_{+}$stands for $\max \{\xi, 0\}$. Indeed, applying the maximum principle yields that $k$ is nonnegative on $\Omega$ (see $[28$, Chap. $4, \S 4.4 .3]$ ). Moreover, it appears later on that, at least in dimension $d=2$, it is bounded. So this choice does not seem restrictive.

From now on, we assume that $\Omega$ is a polygon or a polyhedron. We intend to investigate the regularity properties of the solution $(\boldsymbol{u}, p, k)$ in this case. The idea of the next proof is due to N.G. Meyers [34]. It relies on the well-known property that the solution of the Stokes problem with smooth enough data belongs to $H^{\frac{3}{2}}(\Omega)^{d} \times H^{\frac{1}{2}}(\Omega)$, see $[22, \S 7.3 .3]$ for instance.

Proposition 3.3. There exists a real number $q_{0}>2$ depending on the geometry of $\Omega$ and on the ratio $\nu_{2} / \nu_{0}$ such that, for any $q, 2<q \leq q_{0}$, and for any data $\boldsymbol{f}$ in $W^{-1, q}(\Omega)^{d}$, any solution $(\boldsymbol{u}, p, k)$ of problem (3.3) belongs to $W^{1, q}(\Omega)^{d} \times L^{q}(\Omega) \times W^{2, \frac{q}{2}}(\Omega)$ and satisfies, for a constant $c_{q}$ only depending on $\Omega, q, \nu_{0}$ and $\nu_{2}$,

$$
\nu_{0}\|\boldsymbol{u}\|_{W^{1, q}(\Omega)^{d}}+\|p\|_{L^{q}(\Omega)}+\alpha\|k\|_{W^{2, \frac{q}{2}(\Omega)}} \leq c_{q}\left(\|\boldsymbol{f}\|_{W^{-1, q}(\Omega)^{d}}+\|\boldsymbol{f}\|_{H^{-1}(\Omega)^{d}}^{2} .\right.
$$

Proof: Let $\mathcal{S}_{\sharp}$ denote the Stokes operator, i.e., the operator which associates with any data $\boldsymbol{F}$ in $H^{-1}(\Omega)^{d}$, the part $\boldsymbol{u}$ of the solution $(\boldsymbol{u}, p)$ of the Stokes problem

$$
\begin{cases}-\Delta \boldsymbol{u}+\operatorname{grad} p=\boldsymbol{F} & \text { in } \Omega, \\ \operatorname{div} \boldsymbol{u}=0 & \text { in } \Omega, \\ \boldsymbol{u}=\mathbf{0} & \text { on } \partial \Omega .\end{cases}
$$


When taking $\nu_{02}$ equal to $\frac{\nu_{0}+\nu_{2}}{2}$, we observe that the first two lines in problem (1.2) can equivalently be written as

$$
\boldsymbol{u}+\mathcal{S}_{\sharp}\left(\operatorname{div}\left(\left(1-\frac{\nu(k)}{\nu_{02}}\right) \nabla \boldsymbol{u}\right)\right)=\mathcal{S}_{\sharp}\left(\frac{\boldsymbol{f}-(\boldsymbol{u} \cdot \nabla) \boldsymbol{u}}{\nu_{02}}\right) .
$$

Furthermore, it follows from the Sobolev imbedding theorem that the term $(\boldsymbol{u} \cdot \nabla) \boldsymbol{u}$ belongs to $L^{r}(\Omega)$ for all $r<2$ in dimension $d=2$ and $r \leq \frac{3}{2}$ in dimension $d=3$. Using the fact that $L^{r}(\Omega)$ is imbedded in $W^{-1, r^{*}}(\Omega)$ if and only if the dual space $W_{0}^{1, r^{* \prime}}(\Omega)$ of $W^{-1, r^{*}}(\Omega)$ (with $\frac{1}{r^{*}}+\frac{1}{r^{* \prime}}=1$ ) is imbedded in $L^{r^{\prime}}(\Omega)$ (with $\frac{1}{r}+\frac{1}{r^{\prime}}=1$ ) combined with the Sobolev imbedding theorem, we obtain that $(\boldsymbol{u} \cdot \nabla) \boldsymbol{u}$ belongs to $W^{-1, r^{*}}(\Omega)$ for all $r^{*}<+\infty$ in dimension $d=2$ and $r^{*} \leq 3$ in dimension $d=3$. The same arguments, combined with (3.4), also yield that, for such an $r^{*}$,

$$
\left\|\frac{\boldsymbol{f}-(\boldsymbol{u} \cdot \nabla) \boldsymbol{u}}{\nu_{02}}\right\|_{W^{-1, r^{*}(\Omega)^{d}}} \leq c\left(\|\boldsymbol{f}\|_{W^{-1, r^{*}}(\Omega)^{d}}+\|\boldsymbol{f}\|_{H^{-1}(\Omega)^{d}}^{2}\right) .
$$

Next, we observe that:

1) The Stokes operator $\mathcal{S}_{\sharp}$ is continuous from $H^{-1}(\Omega)^{d}$ into $H_{0}^{1}(\Omega)^{d}$ with norm $\leq 1$ and also from $W^{-1, q_{\sharp}}(\Omega)^{d}$ into $W_{0}^{1, q_{\sharp}}(\Omega)^{d}$ for $q_{\sharp}=6-d$, see [21] or [32] and [33]. If $\chi$ denotes the norm of $\mathcal{S}_{\sharp}$ in the space of linear applications from $W^{-1, q_{\sharp}}(\Omega)^{d}$ into $W_{0}^{1, q_{\sharp}}(\Omega)^{d}$, an interpolation argument (see [31, Chap. 1, Th. 5.1] or [1, Thms $7.17 \& 7.20]$ ) yields that $\mathcal{S}_{\sharp}$ is continuous from $W^{-1, q}(\Omega)^{d}$ into $W_{0}^{1, q}(\Omega)^{d}$ for all $q, 2 \leq q \leq q_{\sharp}$, with norm $\leq \chi^{\theta(q)}$, where $\theta$ is a continuous increasing function on $\left[2, q_{\sharp}\right]$, equal to zero in 2 and to 1 in $q_{\sharp}$ (we do not make it precise for simplicity).

2) For any $q, 2 \leq q \leq \infty$, the norm of the divergence operator from $L^{q}(\Omega)^{d \times d}$ into $W^{-1, q}(\Omega)^{d}$ is $\leq 1$, and the same property holds for the norm of the gradient operator from $W^{1, q}(\Omega)^{d}$ into $L^{q}(\Omega)^{d \times d}$.

3 ) For any $q, 2 \leq q \leq \infty$, and any function $k$, the norm of the multiplication by $1-\frac{\nu(k)}{\nu_{02}}$ from $L^{q}(\Omega)^{d \times d}$ into itself is smaller than $\left|1-\frac{\nu_{2}}{\nu_{02}}\right|=\frac{\nu_{2}-\nu_{0}}{\nu_{2}+\nu_{0}}$.

It follows from the previous arguments that the operator in the left-hand side of (3.8) is an automorphism of $W^{1, q}(\Omega)^{d}$ for all $q \leq q_{\sharp}$ such that

$$
\chi^{\theta(q)} \frac{\nu_{2}-\nu_{0}}{\nu_{2}+\nu_{0}}<1
$$

The existence of a $q_{0}>2$ satisfying this condition comes from the fact that the limit of $\chi^{\theta(q)}$ when $q$ tends to 2 is equal to 1 and that the quantity $\frac{\nu_{2}-\nu_{0}}{\nu_{2}+\nu_{0}}$ is $<1$.

Next, for a $q \leq q_{\sharp}$ satisfying (3.10) and since the right-hand side of (3.8) belongs to $W^{1, q}(\Omega)^{d}$, the velocity $\boldsymbol{u}$ belongs to $W^{1, q}(\Omega)^{d}$ and, owing to (3.9), satisfies (3.6). Then, $\operatorname{grad} p$ obviously belongs to $W^{-1, q}(\Omega)$, so that $p$ belongs to $L^{q}(\Omega)$ and also satisfies (3.6) thanks to the estimate on $\boldsymbol{u}$ and (3.9). Finally, $k$ is the solution of a Laplace equation with right-hand side in $L^{\frac{q}{2}}(\Omega)$, hence belongs to $W^{2, q_{b}}(\Omega)$ with $q_{b}=\min \left\{\frac{q}{2}, \frac{4}{3}\right\}$ (see [22, Thm 4.3.2.4], [15, Th. 2] or [16, Cor. 3.10]) and satisfies (3.6).

Note that the results of Proposition 3.3 can be slightly improved when the domain $\Omega$ is convex or has a smooth boundary. 
Remark 3.4. Similar arguments yield that, if the function $\nu$ belongs to $W^{1, \infty}(\mathbb{R})$ and if the data $\boldsymbol{f}$ are smooth enough, the part $\boldsymbol{u}$ of the solution $(\boldsymbol{u}, p, k)$ of problem (3.3) belongs to $H^{s+1}(\Omega)^{d}$ for a real number $s>0$ also depending on the geometry of $\Omega$ and on the function $\nu$.

We now state and prove a uniqueness result, only in dimension $d=2$. Indeed, its analogue in dimension $d=3$ would require an assumption on the regularity of the solution which is not likely.

Proposition 3.5. Assume that the function $\nu$ is Lipschitz-continuous with Lipschitz constant $\nu_{0}^{*}$. In dimension $d=2$, for any real number $q>2$ and any data $\boldsymbol{f}$ in $W^{-1, q}(\Omega)^{2}$ such that, for appropriate constants $c_{1}^{*}$ and $c_{2}^{*}$,

$$
\frac{c_{1}^{*}}{\nu_{0}^{2}}\left(\|\boldsymbol{f}\|_{W^{-1, q}(\Omega)^{2}}+\|\boldsymbol{f}\|_{H^{-1}(\Omega)^{2}}^{2}\right) \leq 1, \quad \frac{c_{2}^{*} \nu_{0}^{*}}{\alpha \nu_{0}^{2}}\left(\|\boldsymbol{f}\|_{W^{-1, q}(\Omega)^{2}}+\|\boldsymbol{f}\|_{H^{-1}(\Omega)^{2}}^{2}\right)^{2} \leq 1
$$

problem (3.3) admits at most a solution $(\boldsymbol{u}, p, k)$ in $H_{0}^{1}(\Omega)^{2} \times L_{0}^{2}(\Omega) \times W_{0}^{1, r^{\prime}}(\Omega)$.

Proof: Without restriction, we assume that $q \leq q_{0}$ for the $q_{0}$ introduced in Proposition 3.3 , and we denote by $c_{q}(\boldsymbol{f})$ the quantity (which appears in estimate (3.6))

$$
c_{q}(\boldsymbol{f})=c_{q}\left(\|f\|_{W^{-1, q}(\Omega)^{2}}+\|f\|_{H^{-1}(\Omega)^{2}}^{2}\right) .
$$

Let $\left(\boldsymbol{u}_{1}, p_{1}, k_{1}\right)$ and $\left(\boldsymbol{u}_{2}, p_{2}, k_{2}\right)$ be two solutions of problem (3.3). We set: $\boldsymbol{u}=\boldsymbol{u}_{1}-\boldsymbol{u}_{2}$, $p=p_{1}-p_{2}, k=k_{1}-k_{2}$. Next, we proceed in three steps.

1) The pair $(\boldsymbol{u}, p)$ satisfies

$$
\begin{cases}\begin{array}{l}
-\operatorname{div}\left(\nu\left(k_{1}\right) \nabla \boldsymbol{u}\right)+\operatorname{grad} p \\
=\operatorname{div}\left(\left(\nu\left(k_{1}\right)-\nu\left(k_{2}\right)\right) \nabla \boldsymbol{u}_{2}\right)+\left(\boldsymbol{u}_{2} \cdot \nabla\right) \boldsymbol{u}_{2}-\left(\boldsymbol{u}_{1} \cdot \nabla\right) \boldsymbol{u}_{1}
\end{array} & \text { in } \Omega, \\
\operatorname{div} \boldsymbol{u}=0 & \text { in } \Omega, \\
\boldsymbol{u}=\mathbf{0} & \text { on } \partial \Omega .\end{cases}
$$

Writing these equations in the form (3.8), we derive thanks to the same arguments as for Proposition 3.3

$$
\begin{aligned}
\nu_{0}\|\boldsymbol{u}\|_{W^{1, q}(\Omega)^{2}} \leq c\left(\| \operatorname{div}\left(\left(\nu\left(k_{1}\right)-\right.\right.\right. & \left.\left.\nu\left(k_{2}\right)\right) \nabla \boldsymbol{u}_{2}\right) \|_{W^{-1, q}(\Omega)^{2}} \\
& \left.+\left\|\left(\boldsymbol{u}_{2} \cdot \nabla\right) \boldsymbol{u}_{2}-\left(\boldsymbol{u}_{1} \cdot \nabla\right) \boldsymbol{u}_{1}\right\|_{W^{-1, q}(\Omega)^{2}}\right) .
\end{aligned}
$$

It follows from the Lipschitz property of $\nu$ that

$$
\left\|\operatorname{div}\left(\left(\nu\left(k_{1}\right)-\nu\left(k_{2}\right)\right) \nabla \boldsymbol{u}_{2}\right)\right\|_{W^{-1, q}(\Omega)^{2}} \leq \nu_{0}^{*}\|k\|_{L^{\infty}(\Omega)}\left\|\nabla \boldsymbol{u}_{2}\right\|_{L^{q}(\Omega)^{2 \times 2}}
$$

Thus, applying estimate (3.6) to $\boldsymbol{u}_{2}$ and using the Sobolev imbedding of $W^{2, \frac{q}{2}}(\Omega)$ into $L^{\infty}(\Omega)$, we derive

$$
\left\|\operatorname{div}\left(\left(\nu\left(k_{1}\right)-\nu\left(k_{2}\right)\right) \nabla \boldsymbol{u}_{2}\right)\right\|_{W^{-1, q}(\Omega)^{2}} \leq c \frac{\nu_{0}^{*}}{\nu_{0}} c_{q}(\boldsymbol{f})\|k\|_{W^{2, \frac{q}{2}}(\Omega)} .
$$


Similarly, we have

$$
\left\|\left(\boldsymbol{u}_{2} \cdot \nabla\right) \boldsymbol{u}_{2}-\left(\boldsymbol{u}_{1} \cdot \nabla\right) \boldsymbol{u}_{1}\right\|_{W^{-1, q}(\Omega)^{2}} \leq c \frac{2}{\nu_{0}} c_{q}(\boldsymbol{f})\|\boldsymbol{u}\|_{W^{1, q}(\Omega)^{2}}
$$

Combining all this yields

$$
\nu_{0}\|\boldsymbol{u}\|_{W^{1, q}(\Omega)^{2}} \leq c \frac{\nu_{0}^{*}}{\nu_{0}} c_{q}(\boldsymbol{f})\|k\|_{W^{2, \frac{q}{2}}(\Omega)}+c \frac{2}{\nu_{0}} c_{q}(\boldsymbol{f})\|\boldsymbol{u}\|_{W^{1, q}(\Omega)^{2}} .
$$

Thus, the following estimate follows from the first condition in (3.11) (with $c_{1}^{*}=4 c c_{q}$ )

$$
\frac{\nu_{0}}{2}\|\boldsymbol{u}\|_{W^{1, q}(\Omega)^{2}} \leq c \frac{\nu_{0}^{*}}{\nu_{0}} c_{q}(\boldsymbol{f})\|k\|_{W^{2, \frac{q}{2}}(\Omega)} .
$$

2) The function $k$ is a solution of the Laplace equation

$$
\begin{cases}-\alpha \Delta k=\nu\left(k_{1}\right)\left|\nabla \boldsymbol{u}_{1}\right|^{2}-\nu\left(k_{2}\right)\left|\nabla \boldsymbol{u}_{2}\right|^{2} & \text { in } \Omega, \\ k=0 & \text { on } \partial \Omega .\end{cases}
$$

So, similar arguments lead to the estimates

$$
\alpha\|k\|_{W^{2, \frac{q}{2}(\Omega)}} \leq \frac{c^{\prime} \nu_{0}^{*}}{\nu_{0}^{2}} c_{q}(\boldsymbol{f})^{2}\|k\|_{W^{2, \frac{q}{2}(\Omega)}}+\frac{2 c^{\prime} \nu_{2}}{\nu_{0}} c_{q}(\boldsymbol{f})\|\boldsymbol{u}\|_{W^{1, q}(\Omega)^{2}} .
$$

So, the second condition in $(3.11)$ (with $c_{2}^{*} \geq c_{2}=2 c^{\prime} c_{q}^{2}$ ) yields

$$
\frac{\alpha}{2}\|k\|_{W^{2, \frac{q}{2}}(\Omega)} \leq \frac{2 c^{\prime} \nu_{2}}{\nu_{0}} c_{q}(\boldsymbol{f})\|\boldsymbol{u}\|_{W^{1, q}(\Omega)^{2}} .
$$

3) Combining (3.13) and (3.14) leads to

$$
\frac{\nu_{0}}{2}\|\boldsymbol{u}\|_{W^{1, q}(\Omega)^{2}} \leq c \frac{\nu_{0}^{*}}{\nu_{0}} c_{q}(\boldsymbol{f}) \frac{4 c^{\prime} \nu_{2}}{\alpha \nu_{0}} c_{q}(\boldsymbol{f})\|\boldsymbol{u}\|_{W^{1, q}(\Omega)^{2}}
$$

When the second condition in (3.11) holds (with $\left.c_{2}^{*}=\max \left\{c_{2}, 16 c c^{\prime} c_{q}^{2} \frac{\nu_{2}}{\nu_{0}}\right\}\right), \boldsymbol{u}$ is zero. Thus, it follows from (3.14) that $k$ is zero and from the equation

$$
\forall \boldsymbol{v} \in H_{0}^{1}(\Omega)^{2}, \quad \int_{\Omega}(\operatorname{div} \boldsymbol{v})(\boldsymbol{x}) p(\boldsymbol{x}) d \boldsymbol{x}=0,
$$

combined with the already quoted inf-sup condition on this form, that $p$ is zero. This concludes the proof.

The first condition in (3.11) with $q=2$ is very similar to the condition which is sufficient for the uniqueness of the solution of Navier-Stokes equations, see [20, Chap. IV, Thm 2.2]. Still more than for the Navier-Stokes equations, the conditions in (3.11) are too restrictive and will not be assumed in what follows. Moreover it can be noted that the function $\nu$ defined in (3.5) is not Lipschitz-continuous. However and without restriction, we can replace this function by, for a fixed positive parameter $\varepsilon$,

$$
\forall \xi \in \mathbb{R}, \quad \nu(\xi)=\min \left\{\nu_{0}+\nu_{1} \sqrt{\xi_{\varepsilon+}}, \nu_{2}\right\},
$$

where $\xi_{\varepsilon+}$ stands for $\max \{\xi, \varepsilon\}$, in order to recover the Lipschitz property. 


\section{The reduced problem.}

We now assume that the domain $\Omega$ admits a partition into $\Omega_{t}$ and $\Omega_{\ell}$ satisfying (2.1). We suppose that both $\Omega_{t}$ and $\Omega_{\ell}$ are polygons or polyhedra with Lipschitz-continuous boundaries. The strategy for an automatic determination of $\Omega_{t}$ and $\Omega_{\ell}$ is described in Section 2.

We also assume that the function $\nu$ is given by (3.15). We introduce a modified viscosity function $\nu^{*}$ defined by

$$
\forall \xi \in \mathbb{R}, \quad \nu^{*}(\boldsymbol{x}, \xi)= \begin{cases}\nu(\xi)=\min \left\{\nu_{0}+\nu_{1} \sqrt{\xi_{\varepsilon+}}, \nu_{2}\right\} & \text { for a.e. } \boldsymbol{x} \text { in } \Omega_{t} \\ \nu_{0} & \text { for a.e. } \boldsymbol{x} \text { in } \Omega_{\ell}\end{cases}
$$

i.e.,

$$
\forall \boldsymbol{x} \in \Omega, \forall \xi \in \mathbb{R}, \quad \nu^{*}(\boldsymbol{x}, \xi)=\chi_{\Omega_{t}}(\boldsymbol{x}) \nu(\xi)+\chi_{\Omega_{\ell}}(\boldsymbol{x}) \nu_{0},
$$

where $\chi_{\Omega_{t}}$ and $\chi_{\Omega_{\ell}}$ stand for the characteristic functions of $\Omega_{t}$ and $\Omega_{\ell}$, respectively. Next, we consider the reduced problem

$$
\begin{cases}-\operatorname{div}\left(\nu^{*}\left(\cdot, k^{*}\right) \nabla \boldsymbol{u}^{*}\right)+\left(\boldsymbol{u}^{*} \cdot \nabla\right) \boldsymbol{u}^{*}+\operatorname{grad} p^{*}=\boldsymbol{f} & \text { in } \Omega, \\ \operatorname{div} \boldsymbol{u}^{*}=0 & \text { in } \Omega, \\ -\alpha \Delta k^{*}=\nu^{*}\left(\cdot, k^{*}\right)\left|\nabla \boldsymbol{u}^{*}\right|^{2} & \text { in } \Omega, \\ \boldsymbol{u}^{*}=\mathbf{0} & \text { on } \partial \Omega, \\ k^{*}=0 & \text { on } \partial \Omega .\end{cases}
$$

The same arguments as in Section 3 imply that this problem admits the equivalent variational formulation, for some real number $r>d$ and with $\frac{1}{r}+\frac{1}{r^{\prime}}=1$,

Find $\left(\boldsymbol{u}^{*}, p^{*}, k^{*}\right)$ in $H_{0}^{1}(\Omega)^{d} \times L_{0}^{2}(\Omega) \times W_{0}^{1, r^{\prime}}(\Omega)$ such that

$$
\begin{aligned}
& \forall \boldsymbol{v} \in H_{0}^{1}(\Omega)^{d}, \quad \int_{\Omega} \nu^{*}\left(\boldsymbol{x}, k^{*}\right) \nabla \boldsymbol{u}^{*}: \nabla \boldsymbol{v} d \boldsymbol{x}+\int_{\Omega}\left(\boldsymbol{u}^{*} \cdot \nabla\right) \boldsymbol{u}^{*} \cdot \boldsymbol{v}(\boldsymbol{x}) d \boldsymbol{x} \\
& -\int_{\Omega}(\operatorname{div} \boldsymbol{v})(\boldsymbol{x}) p^{*}(\boldsymbol{x}) d \boldsymbol{x}=\langle\boldsymbol{f}, \boldsymbol{v}\rangle, \\
& \forall q \in L_{0}^{2}(\Omega), \quad-\int_{\Omega}\left(\operatorname{div} \boldsymbol{u}^{*}\right)(\boldsymbol{x}) q(\boldsymbol{x}) d \boldsymbol{x}=0, \\
& \forall \chi \in W_{0}^{1, r}(\Omega), \quad \alpha \int_{\Omega} \operatorname{grad} k^{*} \cdot \operatorname{grad} \chi d \boldsymbol{x}=\int_{\Omega} \nu^{*}\left(\boldsymbol{x}, k^{*}\right)\left|\nabla \boldsymbol{u}^{*}\right|^{2} \chi(\boldsymbol{x}) d \boldsymbol{x} .
\end{aligned}
$$

The a priori estimates on the solution $\left(\boldsymbol{u}^{*}, p^{*}, k^{*}\right)$ are also easily derived from the fact that the function $\nu^{*}$ is bounded from above and from below by the same constants as $\nu$. 
Proposition 4.1. For any data $\boldsymbol{f}$ in $H^{-1}(\Omega)^{d}$, any solution $\left(\boldsymbol{u}^{*}, p^{*}, k^{*}\right)$ of problem (4.4) satisfies the following bound

$$
\begin{gathered}
\nu_{0}\left\|\boldsymbol{u}^{*}\right\|_{H^{1}(\Omega)^{d}} \leq c\|\boldsymbol{f}\|_{H^{-1}(\Omega)^{d}}, \\
\left\|p^{*}\right\|_{L^{2}(\Omega)} \leq c\left(\left(1+\frac{\nu_{2}}{\nu_{0}}\right)\|\boldsymbol{f}\|_{H^{-1}(\Omega)^{d}}+\frac{1}{\nu_{0}^{2}}\|\boldsymbol{f}\|_{H^{-1}(\Omega)^{d}}^{2}\right), \\
\alpha\left\|k^{*}\right\|_{W^{1, r^{\prime}}(\Omega)} \leq c \frac{\nu_{2}}{\nu_{0}^{2}}\|\boldsymbol{f}\|_{H^{-1}(\Omega)^{d}}^{2},
\end{gathered}
$$

for a constant $c$ only depending on $\Omega$ and $r$.

The function $\nu^{*}$ is no longer continuous on $\Omega$, however it belongs to $L^{\infty}(\Omega \times \mathbb{R})$ and even to $L^{\infty}\left(\Omega ; \mathscr{C}^{0}(\mathbb{R})\right)$. So a direct extension of the arguments in [29] leads to the existence result.

Theorem 4.2. For any data $\boldsymbol{f}$ in $H^{-1}(\Omega)^{d}$, problem (4.4) admits a solution $\left(\boldsymbol{u}^{*}, p^{*}, k^{*}\right)$ in $H_{0}^{1}(\Omega)^{d} \times L_{0}^{2}(\Omega) \times W_{0}^{1, r^{\prime}}(\Omega)$. Moreover this solution satisfies (4.5).

Note that the arguments used for the proof of Proposition 3.3 only requires the boundedness of $\nu$. So the results of this proposition still hold with $\nu$ replaced by $\nu^{*}$.

Proposition 4.3. For the real number $q_{0}>2$ introduced in Proposition 3.3, for any $q$, $2<q \leq q_{0}$, and for any data $\boldsymbol{f}$ in $W^{-1, q}(\Omega)^{d}$, any solution $\left(\boldsymbol{u}^{*}, p^{*}, k^{*}\right)$ of problem (4.4) belongs to $W^{1, q}(\Omega)^{d} \times L^{q}(\Omega) \times W^{2, \frac{q}{2}}(\Omega)$ and satisfies $(3.6)$.

We now intend to prove a first a posteriori estimate concerning the distance between $(\boldsymbol{u}, p, k)$ and $\left(\boldsymbol{u}^{*}, p^{*}, k^{*}\right)$. To do this, we write both problems (3.3) and (4.4) in a different formulation, which is also useful for the numerical analysis of the discretization. Let $\mathcal{S}$ denote the Stokes operator, i.e., the operator which associates with any data $\boldsymbol{F}$ in $H^{-1}(\Omega)^{d}$, the part $\boldsymbol{u}$ of the solution $(\boldsymbol{u}, p)$ of the Stokes-like problem (the viscosity $\nu_{0}$ corresponds to the case where $\Omega_{t}$ is empty)

$$
\begin{cases}-\nu_{0} \Delta \boldsymbol{u}+\operatorname{grad} p=\boldsymbol{F} & \text { in } \Omega, \\ \operatorname{div} \boldsymbol{u}=0 & \text { in } \Omega, \\ \boldsymbol{u}=\mathbf{0} & \text { on } \partial \Omega,\end{cases}
$$

and similarly let $\mathcal{L}$ denote the inverse Laplace operator, i.e., the operator which associates with any data $G$ in $W^{-1, r^{\prime}}(\Omega)$, the solution $k$ of the Laplace equation

$$
\begin{cases}-\alpha \Delta k=G & \text { in } \Omega, \\ k=0 & \text { on } \partial \Omega .\end{cases}
$$

For a real number $\rho$ which is made precise later on, we set:

$$
\mathcal{X}=W_{0}^{1, \rho}(\Omega)^{d} \times W_{0}^{1, \rho}(\Omega),
$$

and assume that $\boldsymbol{f}$ belongs to $W^{-1, \rho}(\Omega)^{d}$. Thus it follows from Proposition 3.3 that, for appropriate values of $\rho$, problem (3.3) can equivalently be written as: Find $U=(\boldsymbol{u}, k)^{\mathrm{T}}$ in $\mathcal{X}$ such that

$$
\mathcal{F}(U)=U+\left(\begin{array}{ll}
\mathcal{S} & 0 \\
0 & \mathcal{L}
\end{array}\right) \mathcal{G}(U)=0
$$


where $\mathcal{G}$ is given by

$$
\mathcal{G}(U)=\left(\begin{array}{c}
\operatorname{div}\left(\left(\nu_{0}-\nu(k)\right) \nabla \boldsymbol{u}\right)+(\boldsymbol{u} \cdot \nabla) \boldsymbol{u}-\boldsymbol{f} \\
-\nu(k)|\nabla \boldsymbol{u}|^{2}
\end{array}\right)
$$

In an analogous way, problem (4.4) can equivalently be written as: Find $U^{*}=\left(\boldsymbol{u}^{*}, k^{*}\right)^{\mathrm{T}}$ in $\mathcal{X}$ such that

$$
\mathcal{F}_{*}\left(U^{*}\right)=U^{*}+\left(\begin{array}{cc}
\mathcal{S} & 0 \\
0 & \mathcal{L}
\end{array}\right) \mathcal{G}_{*}\left(U^{*}\right)=0
$$

where $\mathcal{G}_{*}$ is given by

$$
\mathcal{G}_{*}(U)=\left(\begin{array}{c}
\operatorname{div}\left(\left(\nu_{0}-\nu^{*}(\cdot, k)\right) \nabla \boldsymbol{u}\right)+(\boldsymbol{u} \cdot \nabla) \boldsymbol{u}-\boldsymbol{f} \\
-\nu^{*}(\cdot, k)|\nabla \boldsymbol{u}|^{2}
\end{array}\right) .
$$

Let $D$ denote the differentiation operator with respect to $U$. In what follows, we are led to assume that $D \mathcal{F}(U)$ is an isomorphism of $\mathcal{X}$. So, we consider the following problem, for any data $(\boldsymbol{\Phi}, \Psi)$ in $H^{-1}(\Omega)^{d} \times W^{-1, r^{\prime}}(\Omega)$,

Find $(\boldsymbol{w}, \pi, \kappa)$ in $H_{0}^{1}(\Omega)^{d} \times L_{0}^{2}(\Omega) \times W_{0}^{1, r^{\prime}}(\Omega)$ such that

$$
\begin{array}{r}
\forall \boldsymbol{v} \in H_{0}^{1}(\Omega)^{d}, \quad \int_{\Omega} \nu(k) \nabla \boldsymbol{w}: \nabla \boldsymbol{v} d \boldsymbol{x}+\int_{\Omega} \nu^{\prime}(k) \kappa \nabla \boldsymbol{u}: \nabla \boldsymbol{v} d \boldsymbol{x} \\
+\int_{\Omega}(\boldsymbol{u} \cdot \nabla) \boldsymbol{w} \cdot \boldsymbol{v}(\boldsymbol{x}) d \boldsymbol{x}+\int_{\Omega}(\boldsymbol{w} \cdot \nabla) \boldsymbol{u} \cdot \boldsymbol{v}(\boldsymbol{x}) d \boldsymbol{x} \\
\quad-\int_{\Omega}(\operatorname{div} \boldsymbol{v})(\boldsymbol{x}) \pi(\boldsymbol{x}) d \boldsymbol{x}=\langle\boldsymbol{\Phi}, \boldsymbol{v}\rangle,
\end{array}
$$

$\forall q \in L_{0}^{2}(\Omega), \quad-\int_{\Omega}(\operatorname{div} \boldsymbol{w})(\boldsymbol{x}) q(\boldsymbol{x}) d \boldsymbol{x}=0$,

$\forall \chi \in W_{0}^{1, r}(\Omega), \quad \alpha \int_{\Omega} \operatorname{grad} \kappa \cdot \operatorname{grad} \chi d \boldsymbol{x}=\langle\Psi, \chi\rangle$

$$
+2 \int_{\Omega} \nu(k) \nabla \boldsymbol{u} \cdot \nabla \boldsymbol{w} \chi(\boldsymbol{x}) d \boldsymbol{x}+\int_{\Omega} \nu^{\prime}(k) \kappa|\nabla \boldsymbol{u}|^{2} \chi(\boldsymbol{x}) d \boldsymbol{x} .
$$

This problem makes sense when the mappings

$$
\boldsymbol{v} \mapsto \int_{\Omega} \nu^{\prime}(k) \kappa \nabla \boldsymbol{u}: \nabla \boldsymbol{v} d \boldsymbol{x} \quad \text { and } \quad \chi \mapsto \int_{\Omega} \nu^{\prime}(k) \kappa|\nabla \boldsymbol{u}|^{2} \chi(\boldsymbol{x}) d \boldsymbol{x},
$$

are linear continuous forms on $H_{0}^{1}(\Omega)^{d}$ and $W^{1, r}(\Omega)$, respectively. Since $W^{1, r}(\Omega)$ with $r>d$ is imbedded in $L^{\infty}(\Omega)$ and $\nu^{\prime}$ is bounded, this holds when $\kappa \nabla \boldsymbol{u}$ belongs to $L^{2}(\Omega)^{d \times d}$. It can be checked that this property is satisfied for any $(\boldsymbol{u}, \kappa)$ in $W^{1, q}(\Omega)^{3} \times W^{2, \frac{q}{2}}(\Omega)$ when $q>2$ in dimension $d=2$ and $q \geq \frac{12}{5}$ in dimension $d=3$.

As a consequence, when taking $\rho>2$ (for instance) in dimension $d=2$ and $\rho \geq \frac{12}{5}$ in dimension $d=3$, the assumption that $D \mathcal{F}(U)$ is an isomorphism of $\mathcal{X}$ can equivalently be written as follows: For any data $(\boldsymbol{\Phi}, \Psi)$ in $W^{-1, \rho}(\Omega)^{d} \times W^{-1, \rho}(\Omega)$, problem (4.13) has a unique solution and this solution belongs to $W^{1, \rho}(\Omega)^{d} \times L^{\rho}(\Omega) \times W^{1, \rho}(\Omega)$. It can be noted 
that this assumption only requires the local uniqueness of the solution $(\boldsymbol{u}, p, k)$ and it is well-known to be less restrictive than the conditions for its global uniqueness as given in Proposition 3.5 (see [20, Chap. IV, §3.2] for the analogue for the Navier-Stokes equations).

We also need that the mapping: $V \mapsto D \mathcal{F}(V)$ is Lipschitz-continuous at least in a neighbourhood of $U$. This requires for instance that the mapping:

$$
k \mapsto \int_{\Omega} \nu^{\prime}(k) \kappa \nabla \boldsymbol{u}: \nabla \boldsymbol{v} d \boldsymbol{x}
$$

is Lipschitz-continuous or, equivalently that the product $\nu^{\prime \prime}(k) k \kappa \nabla \boldsymbol{u}: \nabla \boldsymbol{v}$ is integrable for all $\boldsymbol{u}$ in $W^{1, q}(\Omega)^{d}$ and $\boldsymbol{v}$ in $H^{1}(\Omega)^{d}, k$ and $\kappa$ in $W^{1, q}(\Omega)$. This is true when $\nu^{\prime \prime}$ is bounded and the product $k \kappa$ belongs to $L^{t}(\Omega)$ with $\frac{1}{t}+\frac{1}{q}=\frac{1}{2}$. This property is always valid in dimension $d=2$ since $W^{1, q}(\Omega)$ is imbedded in $L^{\infty}(\Omega)$ for all $q>2$, but only holds when $q \geq \frac{18}{7}$ in dimension $d=3$. For simplicity, we have decided to make the next hypothesis.

Assumption 4.4. In the case of dimension $d=3$, there exists a real number $\tilde{q}_{0} \geq 3$ such that, for any $q, 2<q \leq \tilde{q}_{0}$, and for any data $\boldsymbol{f}$ in $W^{-1, q}(\Omega)^{d}$, any solution of problem (3.3) or (4.4) belongs to $W^{1, q}(\Omega)^{d} \times L^{q}(\Omega) \times W^{1, q}(\Omega)$.

When looking at (3.10), we observe that this assumption is satisfied at least when the ratio $\nu_{2} / \nu_{0}$ is small enough. So, from now on, we take

$$
2<\rho<q_{0} \quad \text { in dimension } d=2 \quad \text { and } \quad \rho=3 \quad \text { in dimension } d=3 \text {. }
$$

We are now in a position to prove the a posteriori estimate. This relies on a result due to J. Pousin and J. Rappaz [38] (see also [41, §2.1] for another statement). We introduce the function $\mu$ defined by

$$
\forall \xi \in \mathbb{R}, \quad \mu(\xi)=\min \left\{\nu_{1} \sqrt{\xi_{\varepsilon+}}, \nu_{2}-\nu_{0}\right\}
$$

and, in analogy with (4.2), the function $\mu^{*}$ defined by

$$
\forall \boldsymbol{x} \in \Omega, \forall \xi \in \mathbb{R}, \quad \mu^{*}(\boldsymbol{x}, \xi)=\chi_{\Omega_{\ell}}(\boldsymbol{x}) \mu(\xi)
$$

Proposition 4.5. If the function $\nu$ belongs to $W^{2, \infty}(\mathbb{R})$ and Assumption 4.4 is satisfied, let $(\boldsymbol{u}, p, k)$ be a solution of problem (3.3) such that $D \mathcal{F}(U)$, with $U=(\boldsymbol{u}, k)^{\mathrm{T}}$, is an isomorphism of $\mathcal{X}$. There exists a positive number $R$ only depending on this solution such that the following a posteriori error estimate holds

$$
\begin{aligned}
& \left\|\boldsymbol{u}-\boldsymbol{u}^{*}\right\|_{W^{1, \rho}(\Omega)^{d}}+\left\|k-k^{*}\right\|_{W^{1, \rho}(\Omega)} \\
& \quad \leq c\left(\left\|\operatorname{div}\left(\mu^{*}\left(\cdot, k^{*}\right) \nabla \boldsymbol{u}^{*}\right)\right\|_{W^{-1, \rho}(\Omega)^{d}}+\left\|\mu^{*}\left(\cdot, k^{*}\right)\left|\nabla \boldsymbol{u}^{*}\right|^{2}\right\|_{W^{-1, \rho}(\Omega)}\right) .
\end{aligned}
$$

for any solution $\left(\boldsymbol{u}^{*}, p^{*}, k^{*}\right)$ of problem (4.4) such that $\left(\boldsymbol{u}^{*}, k^{*}\right)$ belongs to the ball of $\mathcal{X}$ with centre $(\boldsymbol{u}, k)$ and radius $R$. 
Proof: Since $\nu$ belongs to $W^{2, \infty}(\mathbb{R})$, it follows from Assumption 4.4 and the choice (4.14) of $\rho$ that the mapping $\mathcal{F}$ is continuously differentiable on $\mathcal{X}$ and moreover that the mapping: $V \mapsto D \mathcal{F}(V)$ is Lipschitz-continuous with values in the space of linear endomorphisms of $\mathcal{X}$. Let $\lambda$ denote the corresponding Lipschitz constant. Applying [41, Prop. 2.1] yields that there exist positive constants $R$ and $c$ only depending on the norm of $D \mathcal{F}(U)^{-1}$ and $\lambda$ such that the following estimate holds for any solution $U^{*}$ of problem (4.11) satisfying $\left\|U-U^{*}\right\|_{\mathcal{X}} \leq R$,

$$
\left\|U-U^{*}\right\|_{\mathcal{X}} \leq c\left\|\mathcal{F}\left(U^{*}\right)\right\|_{\mathcal{X}}=c\left\|\mathcal{F}\left(U^{*}\right)-\mathcal{F}_{*}\left(U^{*}\right)\right\|_{\mathcal{X}}
$$

To evaluate this last quantity, we observe that

$$
\mathcal{F}\left(U^{*}\right)-\mathcal{F}_{*}\left(U^{*}\right)=-\left(\begin{array}{cc}
\mathcal{S} & 0 \\
0 & \mathcal{L}
\end{array}\right)\left(\begin{array}{c}
\operatorname{div}\left(\left(\nu\left(k^{*}\right)-\nu^{*}\left(\cdot, k^{*}\right)\right) \nabla \boldsymbol{u}^{*}\right) \\
\left(\nu\left(k^{*}\right)-\nu^{*}\left(\cdot, k^{*}\right)\right)\left|\nabla \boldsymbol{u}^{*}\right|^{2}
\end{array}\right)
$$

Since $\mathcal{S}$ maps $W^{-1, \rho}(\Omega)^{d}$ into $W^{1, \rho}(\Omega)^{d}$ (see [32] and [33] in dimension $d=3$ ) and $\mathcal{L}$ maps $W^{-1, \rho}(\Omega)$ into $W^{1, \rho}(\Omega)$ (see $[16$, Thm 1.1$]$ ), we derive

$$
\begin{aligned}
\| U & -U^{*} \|_{\mathcal{X}} \\
& \leq c\left(\left\|\operatorname{div}\left(\nu\left(k^{*}\right)-\nu^{*}\left(\cdot, k^{*}\right)\right) \nabla \boldsymbol{u}^{*}\right\|_{W^{-1, \rho}(\Omega)^{d}}+\left\|\left(\nu\left(k^{*}\right)-\nu^{*}\left(\cdot, k^{*}\right)\right)\left|\nabla \boldsymbol{u}^{*}\right|^{2}\right\|_{W^{-1, \rho}(\Omega)}\right) .
\end{aligned}
$$

The function $\nu\left(k^{*}\right)-\nu^{*}\left(\cdot, k^{*}\right)$ is equal to $\min \left\{\nu_{1} \sqrt{k_{\varepsilon+}^{*}}, \nu_{2}-\nu_{0}\right\}=\mu\left(k^{*}\right)$ on its support which is contained in $\bar{\Omega}_{\ell}$. So this gives the desired result.

In the previous proposition, we have made the assumption that the solution $\left(\boldsymbol{u}^{*}, p^{*}, k^{*}\right)$ of problem (4.4) is not too far from $(\boldsymbol{u}, p, k)$. Since the reduced problem (4.4) obviously coincides with problem (3.3) when $\Omega_{t}$ is equal to $\Omega$, we now state a convergence result which makes this property consistent. For simplicity, we only give an abridged proof of the next proposition.

Proposition 4.6. Let $\left(\Omega_{t n}\right)_{n}$ be an increasing sequence of open polygons or polyhedra with Lipschitz-continuous boundaries such that $\cup_{n \in \mathbb{N}} \Omega_{t n}$ is equal to $\Omega$, and let $\Omega_{\ell n}$ be equal to $\Omega \backslash \bar{\Omega}_{t n}$. Let also $\left(\boldsymbol{u}_{n}, p_{n}, k_{n}\right)$ be a solution of problem (4.4) with $\Omega_{t}=\Omega_{t n}$ and $\Omega_{\ell}=\Omega_{\ell n}$. If the function $\nu$ belongs to $W^{1, \infty}(\mathbb{R})$, Assumption 4.4 holds and the data $\boldsymbol{f}$ belong to $W^{-1, \rho}(\Omega)^{d} \cap H^{s-1}(\Omega)^{d}$ for a real number $s, 0<s<1$, there exists a subsequence $\left(\boldsymbol{u}_{n^{\prime}}, p_{n^{\prime}}, k_{n^{\prime}}\right)_{n^{\prime}}$ which converges to a solution $(\boldsymbol{u}, p, k)$ of problem $(3.3)$ weakly in $W^{1, \rho}(\Omega)^{d} \times L^{\rho}(\Omega) \times W^{1, \rho}(\Omega)$.

Proof: It follows from Proposition 4.3 that the sequence $\left(\boldsymbol{u}_{n}, p_{n}, k_{n}\right)_{n}$ satisfies

$$
\left\|\boldsymbol{u}_{n}\right\|_{W^{1, \rho}(\Omega)^{d}}+\left\|p_{n}\right\|_{L^{\rho}(\Omega)}+\left\|k_{n}\right\|_{W^{1, \rho}(\Omega)} \leq c_{\rho}\left(\|\boldsymbol{f}\|_{W^{-1, \rho}(\Omega)^{d}}+\|\boldsymbol{f}\|_{H^{-1}(\Omega)^{d}}^{2}\right),
$$

and also (see Remark 3.4), for a real number $s_{0}>0$ small enough,

$$
\left\|\boldsymbol{u}_{n}\right\|_{H^{s_{0}+1}(\Omega)^{d}} \leq c_{\rho}\|\boldsymbol{f}\|_{H^{s_{0}-1}(\Omega)^{d}} .
$$

So, there exists a subsequence $\left(\boldsymbol{u}_{n^{\prime}}, p_{n^{\prime}}, k_{n^{\prime}}\right)_{n^{\prime}}$ which converges to $(\boldsymbol{u}, p, k)$ weakly in $W^{1, \rho}(\Omega)^{d} \times L^{\rho}(\Omega) \times W^{1, \rho}(\Omega)$. It follows from compactness arguments that there exists 
another subsequence, still denoted by $\left(\boldsymbol{u}_{n^{\prime}}, p_{n^{\prime}}, k_{n^{\prime}}\right)_{n^{\prime}}$ for simplicity, such that $\left(\boldsymbol{u}_{n^{\prime}}, k_{n^{\prime}}\right)_{n^{\prime}}$ converges to $(\boldsymbol{u}, k)$ strongly in $H^{1}(\Omega)^{d} \times L^{q}(\Omega)$ for an appropriate real number $q>2$. From this, we deduce that

$$
\begin{aligned}
\forall \boldsymbol{v} \in H_{0}^{1}(\Omega)^{d}, \quad \lim _{n^{\prime} \rightarrow+\infty} \int_{\Omega} \nu^{*}\left(\boldsymbol{x}, k_{n^{\prime}}\right) \nabla \boldsymbol{u}_{n^{\prime}}: \nabla \boldsymbol{v} d \boldsymbol{x}=\int_{\Omega} \nu(k) \nabla \boldsymbol{u}: \nabla \boldsymbol{v} d \boldsymbol{x} \\
\forall \chi \in W_{0}^{1, r}(\Omega), \quad \lim _{n^{\prime} \rightarrow+\infty} \int_{\Omega} \nu^{*}\left(\boldsymbol{x}, k_{n^{\prime}}\right)\left|\nabla \boldsymbol{u}_{n^{\prime}}\right|^{2} \chi(\boldsymbol{x}) d \boldsymbol{x}=\int_{\Omega} \nu(k)|\nabla \boldsymbol{u}|^{2} \chi(\boldsymbol{x}) d \boldsymbol{x} .
\end{aligned}
$$

So, when passing to the limit in the problem satisfied by $\left(\boldsymbol{u}_{n}, p_{n}, k_{n}\right)$, it can be checked from the previous convergence properties that $(\boldsymbol{u}, p, k)$ is a solution of problem (3.3).

Note to conclude that the function $\nu$ defined in $(3.15)$ does not belong to $W^{2, \infty}(\mathbb{R})$. More precisely its derivative is continuous everywhere but at the two points $\xi=\varepsilon$ and $\xi=\xi_{0}=\left(\frac{\nu_{2}-\nu_{0}}{\nu_{1}}\right)^{2}$. Without restriction, we now work with a regularization of the function $\nu$ which coincides with $\nu$ except in the neighbourhoods $] \frac{\varepsilon}{2}, \frac{3 \varepsilon}{2}$ [ and $] \xi_{0}-\frac{\varepsilon}{2}, \xi_{0}+\frac{\varepsilon}{2}$ [ of these two points, as illustrated in Figure 1. We do not make precise this regularization for simplicity and still denote the regularized function by $\nu$.

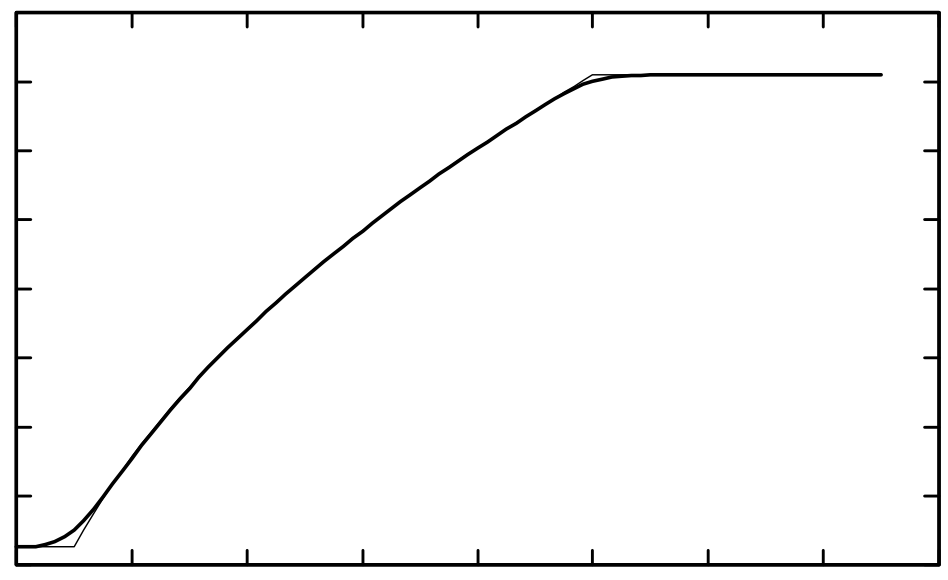

Figure 1. The function $\nu$ and its regularization 


\section{The reduced discrete problem.}

In view of the discretization, we introduce a regular family $\left(\mathcal{T}_{h}\right)_{h}$ of triangulations of $\Omega$ by closed triangles $(d=2)$ or tetrahedra $(d=3)$, in the usual sense that

- for each $h, \bar{\Omega}$ is the union of all elements of $\mathcal{T}_{h}$,

- for each $h$, the intersection of two different elements of $\mathcal{T}_{h}$, if not empty, is a corner, a whole edge or a whole face of both elements,

- the ratio of the diameter $h_{K}$ of an element $K$ in $\mathcal{T}_{h}$ to the diameter of its inscribed circle or sphere is bounded by a constant $\sigma$ independent of $K$ and $h$.

As standard, $h$ denotes the maximum of the diameters of the elements of $\mathcal{T}_{h}$. We make the further and non restrictive assumption that each element $K$ of $\mathcal{T}_{h}$ is contained either in $\bar{\Omega}_{t}$ or in $\bar{\Omega}_{\ell}$. From now on, $c, c^{\prime}, \ldots$ stand for generic constants which may vary from line to line but are always independent of $h$.

For each nonnegative integer $m$ and any $K$ in $\mathcal{T}_{h}$, let $\mathcal{P}_{m}(K)$ denote the space of restrictions to $K$ of polynomials with $d$ variables and total degree $\leq m$. As standard for the Stokes problem, we have decided to work with the Taylor-Hood finite elements, see [26]. Consequently, the discrete spaces of velocities and pressures are defined by

$$
\begin{aligned}
& X_{h}=\left\{\boldsymbol{v}_{h} \in H_{0}^{1}(\Omega)^{d}\right.\left.; \forall K \in \mathcal{T}_{h},\left.\boldsymbol{v}_{h}\right|_{K} \in \mathcal{P}_{2}(K)^{d}\right\}, \\
& M_{h}=\left\{q_{h} \in H^{1}(\Omega) \cap L_{0}^{2}(\Omega) ; \forall K \in \mathcal{T}_{h},\left.q_{h}\right|_{K} \in \mathcal{P}_{1}(K)\right\} .
\end{aligned}
$$

We also use piecewise quadratic functions for approximating the turbulent kinetic energy $k$ in order to preserve the convergence order equal to 2 for the previous elements. So the discrete space of energies is defined by

$$
Y_{h}=\left\{\chi_{h} \in W_{0}^{1, r}(\Omega) ; \forall K \in \mathcal{T}_{h},\left.\chi_{h}\right|_{K} \in \mathcal{P}_{2}(K)\right\}
$$

The discrete problem is then built from (4.4) by the Galerkin method. It reads

Find $\left(\boldsymbol{u}_{h}, p_{h}, k_{h}\right)$ in $X_{h} \times M_{h} \times Y_{h}$ such that

$$
\begin{aligned}
& \forall \boldsymbol{v}_{h} \in X_{h}, \quad \int_{\Omega} \nu^{*}\left(\boldsymbol{x}, k_{h}\right) \nabla \boldsymbol{u}_{h}: \nabla \boldsymbol{v}_{h} d \boldsymbol{x}+\int_{\Omega}\left(\boldsymbol{u}_{h} \cdot \nabla\right) \boldsymbol{u}_{h} \cdot \boldsymbol{v}_{h}(\boldsymbol{x}) d \boldsymbol{x} \\
& -\int_{\Omega}\left(\operatorname{div} \boldsymbol{v}_{h}\right)(\boldsymbol{x}) p_{h}(\boldsymbol{x}) d \boldsymbol{x}=\left\langle\boldsymbol{f}, \boldsymbol{v}_{h}\right\rangle \\
& \forall q_{h} \in M_{h}, \quad-\int_{\Omega}\left(\operatorname{div} \boldsymbol{u}_{h}\right)(\boldsymbol{x}) q_{h}(\boldsymbol{x}) d \boldsymbol{x}=0, \\
& \forall \chi_{h} \in Y_{h}, \quad \alpha \int_{\Omega} \operatorname{grad} k_{h} \cdot \operatorname{grad} \chi_{h} d \boldsymbol{x}=\int_{\Omega} \nu^{*}\left(\boldsymbol{x}, k_{h}\right)\left|\nabla \boldsymbol{u}_{h}\right|^{2} \chi_{h}(\boldsymbol{x}) d \boldsymbol{x} .
\end{aligned}
$$

The imbeddings of $X_{h}$ in $H_{0}^{1}(\Omega)^{d}$, of $M_{h}$ in $L_{0}^{2}(\Omega)$ and of $Y_{h}$ both in $W_{0}^{1, r}(\Omega)$ and in $W_{0}^{1, r^{\prime}}(\Omega)$ yield that the discretization is fully conforming.

Remark 5.1. In the implementation of this problem, $\nu^{*}\left(\cdot, k_{h}\right)$ is replaced by the function $\nu_{h}$ such that, for each $K$ in $\mathcal{T}_{h},\left.\nu_{h}\right|_{K}$ belongs to $\mathcal{P}_{2}(K)$ and which is equal to $\nu^{*}\left(\boldsymbol{a}, k_{h}(\boldsymbol{a})\right)$ at 
each vertex and midpoint $\boldsymbol{a}$ of an edge of $K$. We do not take into account this modification in the analysis for simplicity.

The numerical analysis of this problem relies on the Brezzi-Rappaz-Raviart theorem, see [9] or [20, Chap. IV, Thm 3.1]. We recall that problem (4.4) admits the formulation (4.11); however we are led to use a modified formulation. We now work with $\mathcal{X}$ replaced by the space $\mathcal{Y}$ defined by

$$
\mathcal{Y}=H_{0}^{1}(\Omega)^{d} \times\left(H_{0}^{1}(\Omega) \cap L^{\infty}(\Omega)\right),
$$

and equipped with the corresponding norm, which seems more natural in view of the finite element discretization. For any real number or real-valued measurable function $\xi$ on $\Omega$, we introduce the Stokes operator $\mathcal{S}(\xi)$ which associates with any data $\boldsymbol{F}$ in $H^{-1}(\Omega)^{d}$, the part $\boldsymbol{u}$ of the solution $(\boldsymbol{u}, p)$ of the generalized Stokes problem

$$
\begin{cases}-\operatorname{div}\left(\nu^{*}(\cdot, \xi) \nabla \boldsymbol{u}\right)+\operatorname{grad} p=\boldsymbol{F} & \text { in } \Omega, \\ \operatorname{div} \boldsymbol{u}=0 & \text { in } \Omega, \\ \boldsymbol{u}=\mathbf{0} & \text { on } \partial \Omega .\end{cases}
$$

We also use the operator $\mathcal{L}$ defined in (4.7). Thus, problem (4.4) can equivalently be written as: Find $U^{*}=\left(\boldsymbol{u}^{*}, k^{*}\right)^{\mathrm{T}}$ in $\mathcal{Y}$ such that

$$
\widetilde{\mathcal{F}}\left(U^{*}\right)=U^{*}+\left(\begin{array}{cc}
\mathcal{S}\left(k^{*}\right) & 0 \\
0 & \mathcal{L}
\end{array}\right) \widetilde{\mathcal{G}}\left(U^{*}\right)=0
$$

where $\widetilde{\mathcal{G}}$ is given by

$$
\widetilde{\mathcal{G}}(U)=\left(\begin{array}{c}
(\boldsymbol{u} \cdot \nabla) \boldsymbol{u}-\boldsymbol{f} \\
-\nu^{*}(\cdot, k)|\nabla \boldsymbol{u}|^{2}
\end{array}\right)
$$

Similarly, let $\mathcal{S}_{h}(\xi)$ denote the discrete Stokes operator associated with problem (5.5), i.e., the operator which associates with any data $\boldsymbol{F}$ in $H^{-1}(\Omega)^{d}$, the part $\boldsymbol{u}_{h}$ of the solution $\left(\boldsymbol{u}_{h}, p_{h}\right)$ in $X_{h} \times M_{h}$ of the Stokes problem

$$
\begin{aligned}
& \forall \boldsymbol{v}_{h} \in X_{h}, \quad \int_{\Omega} \nu^{*}(\boldsymbol{x}, \xi) \nabla \boldsymbol{u}_{h}: \nabla \boldsymbol{v}_{h} d \boldsymbol{x}-\int_{\Omega}\left(\operatorname{div} \boldsymbol{v}_{h}\right)(\boldsymbol{x}) p_{h}(\boldsymbol{x}) d \boldsymbol{x}=\left\langle\boldsymbol{F}, \boldsymbol{v}_{h}\right\rangle, \\
& \forall q_{h} \in M_{h}, \quad-\int_{\Omega}\left(\operatorname{div} \boldsymbol{u}_{h}\right)(\boldsymbol{x}) q_{h}(\boldsymbol{x}) d \boldsymbol{x}=0 .
\end{aligned}
$$

Let finally $\mathcal{L}_{h}$ denote the discrete inverse Laplace operator, i.e., the operator which associates with any data $G$ in $H^{-1}(\Omega)$, the solution $k_{h}$ in $Y_{h}$ of the Laplace equation

$$
\forall \chi_{h} \in Y_{h}, \quad \alpha \int_{\Omega} \operatorname{grad} k_{h} \cdot \operatorname{grad} \chi_{h} d \boldsymbol{x}=\left\langle G, \chi_{h}\right\rangle
$$

Indeed, an inf-sup condition on the form: $\left(\boldsymbol{v}_{h}, q_{h}\right) \mapsto-\int_{\Omega}\left(\operatorname{div} \boldsymbol{v}_{h}\right)(\boldsymbol{x}) q_{h}(\boldsymbol{x}) d \boldsymbol{x}$ between the spaces $X_{h}$ and $M_{h}$ is proven for instance in [20, Chap. II, Cor. 4.1], so that these operators are well-defined. Problem (5.3) now admits the equivalent formulation: Find $U_{h}=\left(\boldsymbol{u}_{h}, k_{h}\right)^{\mathrm{T}}$ in $\mathcal{Y}$ such that

$$
\mathcal{F}_{h}\left(U_{h}\right)=U_{h}+\left(\begin{array}{cc}
\mathcal{S}_{h}\left(k_{h}\right) & 0 \\
0 & \mathcal{L}_{h}
\end{array}\right) \widetilde{\mathcal{G}}\left(U_{h}\right)=0
$$


To go further, we recall from a simple extension of [20, Chap. II, Thm 4.3] that the operator $\mathcal{S}_{h}(\xi)$ satisfies the following properties: For any $\boldsymbol{F}$ in $H^{-1}(\Omega)^{d}$,

$$
\left\|\mathcal{S}_{h}(\xi) \boldsymbol{F}\right\|_{H^{1}(\Omega)^{d}} \leq c\|\boldsymbol{F}\|_{H^{-1}(\Omega)^{d}}
$$

and, if moreover $\boldsymbol{F}$ belongs to $H^{s-1}(\Omega)^{d}$ and $\mathcal{S}(\xi) \boldsymbol{F}$ to $H^{s+1}(\Omega)^{d}$ for a real number $s$, $0 \leq s \leq 2$,

$$
\left\|\left(\mathcal{S}(\xi)-\mathcal{S}_{h}(\xi)\right) \boldsymbol{F}\right\|_{H^{1}(\Omega)^{d}} \leq c h^{s}\left(\|\mathcal{S}(\xi) \boldsymbol{F}\|_{H^{s+1}(\Omega)^{d}}+\|\boldsymbol{F}\|_{H^{s-1}(\Omega)^{d}}\right) .
$$

Moreover, since the function $\nu^{*}(\cdot, \xi)$ is bounded from above and from below, both constants in estimates (5.11) and (5.12) are independent of $\xi$. The analogous properties concerning the operator $\mathcal{L}_{h}$ are also standard [6, Chap. X, Th. $\left.1.1 \& 1.2\right]$ : For any data $G$ in $H^{-1}(\Omega)$,

$$
\left\|\mathcal{L}_{h} G\right\|_{H^{1}(\Omega)} \leq c\|G\|_{H^{-1}(\Omega)}
$$

and, if moreover $\mathcal{L} G$ belongs to $H^{s+1}(\Omega)$ for a real number $s, 0 \leq s \leq 2$,

$$
\left\|\left(\mathcal{L}-\mathcal{L}_{h}\right) G\right\|_{H^{1}(\Omega)} \leq c h^{s}\|\mathcal{L} G\|_{H^{s+1}(\Omega)} .
$$

We refer to [36, Cor. 2] for the less usual result: For any data $G$ in $H^{-1}(\Omega)$ such that $\mathcal{L} G$ belongs to $W^{s+1, \infty}(\Omega)$ for $s$ equal to 0,1 or 2 ,

$$
\left\|\left(\mathcal{L}-\mathcal{L}_{h}\right) G\right\|_{L^{\infty}(\Omega)} \leq c h^{s+1}\|\mathcal{L} G\|_{W^{s+1, \infty}(\Omega)} .
$$

Moreover, we are led to assume that, if $\mathcal{L} G$ belongs to $L^{\infty}(\Omega)$,

$$
\left\|\mathcal{L}_{h} G\right\|_{L^{\infty}(\Omega)} \leq c\|\mathcal{L} G\|_{L^{\infty}(\Omega)} .
$$

This property is proved in [39, Thm 5.1] only when the family of triangulations $\left(\mathcal{T}_{h}\right)_{h}$ is uniformly regular. However it seems possible to extend this result to more general families of triangulations.

Let us introduce the matrix operators

$$
\mathcal{M}(\xi)=\left(\begin{array}{cc}
\mathcal{S}(\xi) & 0 \\
0 & \mathcal{L}
\end{array}\right), \quad \mathcal{M}_{h}(\xi)=\left(\begin{array}{cc}
\mathcal{S}_{h}(\xi) & 0 \\
0 & \mathcal{L}_{h}
\end{array}\right)
$$

The following convergence result is easily derived from (5.11) to (5.16) by a density argument: When property (5.16) holds, for any $(\boldsymbol{F}, G)$ in $H^{-1}(\Omega)^{d} \times H^{-1}(\Omega)$ such that $\mathcal{L} G$ belongs to $\mathscr{C}^{0}(\bar{\Omega})$,

$$
\lim _{h \rightarrow 0}\left\|\left(\mathcal{M}(\xi)-\mathcal{M}_{h}(\xi)\right)(\boldsymbol{F}, G)\right\|_{\mathcal{Y}}=0 .
$$

Moreover, we recall from [14, Cor. 18.15] that, since $\Omega$ is a polygon or a polyhedron, the inverse Laplace operator maps $H^{t}(\Omega)$ into $H^{t+2}(\Omega)$ for some real number $t>\frac{d}{2}-2$. Since this $H^{t+2}(\Omega)$ is compactly imbedded in $\mathscr{C}^{0}(\bar{\Omega})$, we deduce from the previous property that

$$
\lim _{h \rightarrow 0} \sup _{(\boldsymbol{F}, G) \in \mathcal{K}}\left\|\left(\mathcal{M}(\xi)-\mathcal{M}_{h}(\xi)\right)(\boldsymbol{F}, G)\right\|_{\mathcal{Y}}=0
$$


for any bounded set $\mathcal{K}$ of the space

$$
\mathcal{Y}^{\diamond}=H^{\tau}(\Omega)^{d} \times H^{t}(\Omega), \quad-1<\tau<0, \frac{d}{2}-2<t<0 .
$$

Let also $\mathcal{E}(\mathcal{Y})$ denote the space of endomorphisms of $\mathcal{Y}$. We are now in a position to prove the following lemmas.

Lemma 5.2. Let $\left(\boldsymbol{u}^{*}, p^{*}, k^{*}\right)$ be a solution of problem (4.4) such that $U^{*}=\left(\boldsymbol{u}^{*}, k^{*}\right)^{\mathrm{T}}$ belongs to $H^{s+1}(\Omega)^{d} \times W^{1, r}(\Omega), s>d-2$ and $r>d$, and that $D \widetilde{\mathcal{F}}\left(U^{*}\right)$ is an isomorphism of $\mathcal{Y}$. When property (5.16) holds, there exists an $h_{0}>0$ such that, for all $h \leq h_{0}$, $D \mathcal{F}_{h}\left(U^{*}\right)$ is an isomorphism of $\mathcal{Y}$ and the norm of its inverse is bounded independently of $h$.

Proof: By writing the identity

$$
D \mathcal{F}_{h}\left(U^{*}\right)=D \widetilde{\mathcal{F}}\left(U^{*}\right)\left(\operatorname{Id}-D \widetilde{\mathcal{F}}\left(U^{*}\right)^{-1}\left(D \widetilde{\mathcal{F}}\left(U^{*}\right)-D \mathcal{F}_{h}\left(U^{*}\right)\right)\right)
$$

we observe that it suffices to check that

$$
\lim _{h \rightarrow 0}\left\|D \widetilde{\mathcal{F}}\left(U^{*}\right)-D \mathcal{F}_{h}\left(U^{*}\right)\right\|_{\mathcal{E}(\mathcal{Y})}=0
$$

We have

$$
D \widetilde{\mathcal{F}}\left(U^{*}\right)-D \mathcal{F}_{h}\left(U^{*}\right)=\left(\mathcal{M}\left(k^{*}\right)-\mathcal{M}_{h}\left(k^{*}\right)\right) D \widetilde{\mathcal{G}}\left(U^{*}\right)+\left(\begin{array}{cc}
D \mathcal{S}\left(k^{*}\right)-D \mathcal{S}_{h}\left(k^{*}\right) & 0 \\
0 & 0
\end{array}\right) \widetilde{\mathcal{G}}\left(U^{*}\right)
$$

and we now prove successively that each term in the right-hand side tends to zero.

1) Thanks to (5.18), it suffices to check that the mapping: $W \mapsto D \widetilde{\mathcal{G}}\left(U^{*}\right) \cdot W$ sends the unit sphere of $\mathcal{Y}$ into a bounded set of $\mathcal{Y}^{\diamond}$. We have, for $W=(\boldsymbol{w}, \kappa)$,

$$
D \widetilde{\mathcal{G}}\left(U^{*}\right) \cdot W=\left(\begin{array}{c}
\left(\boldsymbol{u}^{*} \cdot \nabla\right) \boldsymbol{w}+(\boldsymbol{w} \cdot \nabla) \boldsymbol{u}^{*} \\
-2 \nu^{*}\left(\cdot, k^{*}\right) \nabla \boldsymbol{u}^{*}: \nabla \boldsymbol{w}-\left(\partial_{\xi} \nu^{*}\left(\cdot, k^{*}\right) \kappa\right)\left|\nabla \boldsymbol{u}^{*}\right|^{2}
\end{array}\right)
$$

When $W$ runs though the unit sphere of $\mathcal{Y},\left(\boldsymbol{u}^{*} \cdot \nabla\right) \boldsymbol{w}+(\boldsymbol{w} \cdot \nabla) \boldsymbol{u}^{*}$ belongs to a bounded set of $L^{q}(\Omega)^{d}$ for all $q<2$ in dimension $d=2$ and all $q<\frac{3}{2}$ in dimension $d=3$, and hence to a bounded set of $H^{\tau}(\Omega)^{d}$ for some $\tau,-1<\tau<0$. On the other hand, the two terms in the second component of $D \widetilde{\mathcal{G}}\left(U^{*}\right) \cdot W$ are bounded in $L^{q}(\Omega)$, for all $q$ such that $\frac{1}{q} \geq 1-\frac{s}{d}$ and $\frac{1}{q} \geq 1-\frac{2 s}{d}$, respectively. So their sum belongs to a bounded set of $L^{q}(\Omega)$ with $\frac{1}{q}=1-\frac{s}{d}$, and it follows from the assumption on $s$ that this space is imbedded in $H^{t}(\Omega), t>\frac{d}{2}-2$. By combining this with (5.18), we obtain the convergence result.

2 ) It is readily checked that, for all $\boldsymbol{F}$ in $H^{-1}(\Omega)^{d}$,

$$
\begin{aligned}
\left(D \mathcal{S}\left(k^{*}\right) \kappa\right) \boldsymbol{F}=\mathcal{S}\left(k^{*}\right) & \left(-\operatorname{div}\left(\partial_{\xi} \nu^{*}\left(\cdot, k^{*}\right) \kappa \nabla \mathcal{S}\left(k^{*}\right) \boldsymbol{F}\right)\right), \\
& \left(D \mathcal{S}_{h}\left(k^{*}\right) \kappa\right) \boldsymbol{F}=\mathcal{S}_{h}\left(k^{*}\right)\left(-\operatorname{div}\left(\partial_{\xi} \nu^{*}\left(\cdot, k^{*}\right) \kappa \nabla \mathcal{S}_{h}\left(k^{*}\right) \boldsymbol{F}\right)\right),
\end{aligned}
$$


so that

$$
\begin{gathered}
\left(\begin{array}{cc}
D \mathcal{S}\left(k^{*}\right)-D \mathcal{S}_{h}\left(k^{*}\right) & 0 \\
0 & 0
\end{array}\right) \widetilde{\mathcal{G}}\left(U^{*}\right)=-\left(\begin{array}{c}
\left(\mathcal{S}\left(k^{*}\right)-\mathcal{S}_{h}\left(k^{*}\right)\right) \operatorname{div}\left(\left(\partial_{\xi} \nu^{*}\left(\cdot, k^{*}\right) \kappa\right) \nabla \boldsymbol{u}^{*}\right) \\
0
\end{array}\right) \\
-\left(\begin{array}{cc}
\mathcal{S}_{h}\left(k^{*}\right) & 0 \\
0 & 0
\end{array}\right)\left(\begin{array}{c}
\operatorname{div}\left(\left(\partial_{\xi} \nu^{*}\left(\cdot, k^{*}\right) \kappa\right) \nabla\left(\mathcal{S}\left(k^{*}\right)-\mathcal{S}_{h}\left(k^{*}\right)\right)\left(\left(\boldsymbol{u}^{*} \cdot \nabla\right) \boldsymbol{u}^{*}-\boldsymbol{f}\right)\right) \\
0
\end{array}\right) .
\end{gathered}
$$

There also, the convergence of the first term follows from the fact that $\left(\partial_{\xi} \nu^{*}\left(\cdot, k^{*}\right) \kappa\right) \nabla \boldsymbol{u}^{*}$ belongs to a compact set of $L^{2}(\Omega)^{d \times d}$, hence that $\operatorname{div}\left(\left(\partial_{\xi} \nu^{*}\left(\cdot, k^{*}\right) \kappa\right) \nabla \boldsymbol{u}^{*}\right)$ belongs to a compact set of $H^{-1}(\Omega)^{d}$ when $\kappa$ runs through the unit sphere of $H^{1}(\Omega) \cap L^{\infty}(\Omega)$. The convergence of the second term is an easy consequence of (5.11) and the convergence of $\left(\mathcal{S}\left(k^{*}\right)-\mathcal{S}_{h}\left(k^{*}\right)\right)\left(\left(\boldsymbol{u}^{*} \cdot \nabla\right) \boldsymbol{u}^{*}-\boldsymbol{f}\right)$ in $H^{1}(\Omega)^{d}$.

The stability property (5.16) seems unsufficient in view of what follows. So we now prove two modified results.

Lemma 5.3. The following result holds for any distribution $G$ in $H^{-1}(\Omega)$

$$
\left\|\mathcal{L}_{h} G\right\|_{L^{\infty}(\Omega)} \leq c \lambda_{h}\|G\|_{H^{-1}(\Omega)},
$$

where the quantity $\lambda_{h}$ is defined by

$$
\lambda_{h}=\left\{\begin{array}{ll}
\left|\log h_{\min }\right|^{\frac{1}{2}} & \text { if } d=2, \\
h_{\min }^{-\frac{1}{2}} & \text { if } d=3,
\end{array} \quad \text { with } \quad h_{\min }=\min _{K \in \mathcal{T}_{h}} h_{K} .\right.
$$

Proof: Setting $k_{h}=\mathcal{L}_{h} G$, we define the piecewise constant function $\bar{k}_{h}$ by

$$
\forall K \in \mathcal{T}_{h},\left.\quad \bar{k}_{h}\right|_{K}=\frac{1}{\operatorname{meas}(K)} \int_{K} k_{h}(\boldsymbol{x}) d \boldsymbol{x}
$$

and set: $\tilde{k}_{h}=k_{h}-\bar{k}_{h}$. We now estimate sucessively $\left\|\tilde{k}_{h}\right\|_{L^{\infty}(\Omega)}$ and $\left\|\bar{k}_{h}\right\|_{L^{\infty}(\Omega)}$.

1) We note that

$$
\left\|\tilde{k}_{h}\right\|_{L^{\infty}(\Omega)}=\max _{K \in \mathcal{T}_{h}}\left\|\tilde{k}_{h}\right\|_{L^{\infty}(K)}
$$

By going to a reference element, we have on each $K$ in $\mathcal{T}_{h}$ and with the standard notation

$$
\left\|\tilde{k}_{h}\right\|_{L^{\infty}(K)}=\left\|\hat{\tilde{k}}_{h}\right\|_{L^{\infty}(\hat{K})} \leq c\left\|\hat{\tilde{k}}_{h}\right\|_{H^{1}(\hat{K})}
$$

where we have used the fact that all norms are equivalent on $\mathcal{P}_{2}(\hat{K})$. Since $\tilde{k}_{h}$ has a null integral on $K$, the same property holds for $\hat{\tilde{k}}_{h}$ on $\hat{K}$, so that we derive from the Bramble-Hilbert inequality (see for instance [6, Chap. I, Lemme 2.11])

$$
\left\|\tilde{k}_{h}\right\|_{L^{\infty}(K)} \leq c\left|\hat{\tilde{k}}_{h}\right|_{H^{1}(\hat{K})}
$$

and switching back to $K$ yields

$$
\left\|\tilde{k}_{h}\right\|_{L^{\infty}(K)} \leq c h_{K}^{1-\frac{d}{2}}\left|\tilde{k}_{h}\right|_{H^{1}(K)} .
$$


Noting that $\tilde{k}_{h}-k_{h}$ is constant on each $K$, so that $\left|\tilde{k}_{h}\right|_{H^{1}(K)}$ is equal to $\left|k_{h}\right|_{H^{1}(K)}$, and also that the maximum of the $\left|k_{h}\right|_{H^{1}(K)}$ is bounded by $\left|k_{h}\right|_{H^{1}(\Omega)}$, we obtain

$$
\left\|\tilde{k}_{h}\right\|_{L^{\infty}(\Omega)} \leq c h_{\min }^{1-\frac{d}{2}}\left|k_{h}\right|_{H^{1}(\Omega)} .
$$

2) Let $p$ be a real number $>1$ such that $H^{1}(\Omega)$ is imbedded in $L^{p}(\Omega)$. Setting $\frac{1}{p}+\frac{1}{p^{\prime}}=1$, we have from the definition of $\bar{k}_{h}$ and on each $K$

$$
\left|\bar{k}_{h}\right| \leq \frac{\operatorname{meas}(K)^{\frac{1}{p^{\prime}}}}{\operatorname{meas}(K)}\left\|k_{h}\right\|_{L^{p}(K)} \leq c h_{K}^{-\frac{d}{p}}\left\|k_{h}\right\|_{L^{p}(K)} .
$$

This yields

$$
\left\|\bar{k}_{h}\right\|_{L^{\infty}(\Omega)} \leq c h_{\min }^{-\frac{d}{p}}\left\|k_{h}\right\|_{L^{p}(\Omega)} .
$$

In dimension $d=2$, we recall from [40] that the norm of the imbedding of $H^{1}(\Omega)$ into $L^{p}(\Omega)$ for any $p<+\infty$ behaves like $c \sqrt{p}$ and we take $p=\left|\log h_{\min }\right|$, so that $\sqrt{p} h_{\text {min }}^{-\frac{d}{p}}$ is equal to $e^{2}\left|\log h_{\min }\right|^{\frac{1}{2}}$. In dimension $d=3$ we take $p$ equal to 6 , such that $h_{\min }^{-\frac{d}{p}}$ is equal to $h_{\mathrm{min}}^{-\frac{1}{2}}$. This gives

$$
\left\|\bar{k}_{h}\right\|_{L^{\infty}(\Omega)} \leq c \lambda_{h}\left\|k_{h}\right\|_{H^{1}(\Omega)} .
$$

Finally, it follows from (5.22) and (5.23) that

$$
\left\|k_{h}\right\|_{L^{\infty}(\Omega)} \leq c \lambda_{h}\left\|k_{h}\right\|_{H^{1}(\Omega)},
$$

and combining this result with (5.13) gives the desired estimate.

Lemma 5.4. The following result holds for any distribution $G$ in $L^{1}(\Omega)$

$$
\left\|\mathcal{L}_{h} G\right\|_{H^{1}(\Omega)} \leq c \lambda_{h}\|G\|_{L^{1}(\Omega)}, \quad\left\|\mathcal{L}_{h} G\right\|_{L^{\infty}(\Omega)} \leq c \lambda_{h}^{2}\|G\|_{L^{1}(\Omega)},
$$

where $\lambda_{h}$ is defined in (5.21).

Proof: Owing to (5.24), it suffices to check the first part of (5.25). Setting once more $k_{h}=\mathcal{L}_{h} G$, we observe from the definition $(5.9)$ of $\mathcal{L}_{h}$ and a Poincaré-Friedrichs inequality that

$$
\left\|k_{h}\right\|_{H^{1}(\Omega)}^{2} \leq \frac{c}{\alpha}\|G\|_{L^{1}(\Omega)}\left\|k_{h}\right\|_{L^{\infty}(\Omega)} .
$$

Using once more (5.24) yields the desired estimate.

Lemma 5.5. If the function $\nu$ belongs to $W^{2, \infty}(\mathbb{R})$, the mapping $D \mathcal{F}_{h}$ satisfies the following Lipschitz property, for all $V_{1}$ and $V_{2}$ in a bounded subset of $\mathcal{Y}$,

$$
\left\|D \mathcal{F}_{h}\left(V_{1}\right)-D \mathcal{F}_{h}\left(V_{2}\right)\right\|_{\mathcal{E}(\mathcal{Y})} \leq c \lambda_{h}^{2}\left\|V_{1}-V_{2}\right\|_{\mathcal{Y}}
$$

where $\lambda_{h}$ is defined in (5.21).

Proof: Setting $V_{i}=\left(\boldsymbol{v}_{i}, \ell_{i}\right)$, we have

$$
\begin{aligned}
D \mathcal{F}_{h}\left(V_{1}\right)-D \mathcal{F}_{h}\left(V_{2}\right)=( & \left.\mathcal{M}_{h}\left(\ell_{1}\right)-\mathcal{M}_{h}\left(\ell_{2}\right)\right) D \widetilde{\mathcal{G}}\left(V_{1}\right)+\mathcal{M}_{h}\left(\ell_{2}\right)\left(D \widetilde{\mathcal{G}}\left(V_{1}\right)-D \widetilde{\mathcal{G}}\left(V_{2}\right)\right) \\
& +\left(D \mathcal{M}_{h}\left(\ell_{1}\right)-D \mathcal{M}_{h}\left(\ell_{2}\right)\right) \widetilde{\mathcal{G}}\left(V_{1}\right)+D \mathcal{M}_{h}\left(\ell_{2}\right)\left(\widetilde{\mathcal{G}}\left(V_{1}\right)-\widetilde{\mathcal{G}}\left(V_{2}\right)\right)
\end{aligned}
$$


Let $W=(\boldsymbol{w}, \kappa)$ be any function in $\mathcal{Y}$. The proof now proceeds in two steps. All constants $c$ in what follows only depend on the norms $\left\|V_{1}\right\| \mathcal{Y},\left\|V_{2}\right\|_{\mathcal{Y}}$ and $\|\nu\|_{W^{2, \infty}(\mathbb{R})}$.

1) We have

$$
\begin{aligned}
\left(\mathcal{M}_{h}\left(\ell_{1}\right)\right. & \left.-\mathcal{M}_{h}\left(\ell_{2}\right)\right) D \widetilde{\mathcal{G}}\left(V_{1}\right) W \\
& =\mathcal{M}_{h}\left(\ell_{1}\right)\left(\begin{array}{c}
-\operatorname{div}\left(\left(\nu^{*}\left(\cdot, \ell_{1}\right)-\nu^{*}\left(\cdot, \ell_{2}\right)\right)\right. \\
\left.\nabla \mathcal{S}_{h}\left(\ell_{2}\right)\left(\left(\boldsymbol{v}_{1} \cdot \nabla\right) \boldsymbol{w}+(\boldsymbol{w} \cdot \nabla) \boldsymbol{v}_{1}\right)\right) \\
0
\end{array}\right),
\end{aligned}
$$

which yields in an obvious way

$$
\left\|\left(\mathcal{M}_{h}\left(\ell_{1}\right)-\mathcal{M}_{h}\left(\ell_{2}\right)\right) D \widetilde{\mathcal{G}}\left(V_{1}\right) W\right\|_{\mathcal{Y}} \leq c\left\|V_{1}-V_{2}\right\|_{\mathcal{Y}}\|W\|_{\mathcal{Y}}
$$

On the other hand, since $\partial_{\xi} \nu^{*}(\boldsymbol{x}, \cdot)$ is zero on $\Omega_{\ell}$ while on $\Omega_{t}$ it coincides with $\partial_{\xi} \nu$, it belongs to $W^{1, \infty}(\mathbb{R})$ for a.e. $\boldsymbol{x}$ in $\Omega$. So, combining the second part of (5.19) with a further triangle inequality also yields

$$
\left\|\left(\left(D \mathcal{M}_{h}\left(\ell_{1}\right)-D \mathcal{M}_{h}\left(\ell_{2}\right)\right) \kappa\right) \widetilde{\mathcal{G}}\left(V_{1}\right)\right\|_{\mathcal{Y}} \leq c\left\|V_{1}-V_{2}\right\|_{\mathcal{Y}}\|W\|_{\mathcal{Y}}
$$

2) It follows from (5.19) that

$$
\mathcal{M}_{h}\left(\ell_{2}\right)\left(D \widetilde{\mathcal{G}}\left(V_{1}\right)-D \widetilde{\mathcal{G}}\left(V_{2}\right)\right)+D \mathcal{M}_{h}\left(\ell_{2}\right)\left(\widetilde{\mathcal{G}}\left(V_{1}\right)-\widetilde{\mathcal{G}}\left(V_{2}\right)\right)=\mathcal{M}_{h}\left(\ell_{2}\right)\left(\begin{array}{l}
\Gamma_{\mathcal{S}} \\
\Gamma_{\mathcal{L}}
\end{array}\right)
$$

where

$$
\begin{aligned}
&\left\langle\Gamma_{\mathcal{S}}, W\right\rangle= \begin{array}{r}
\left(\left(\boldsymbol{v}_{1}-\boldsymbol{v}_{2}\right) \cdot \nabla\right) \boldsymbol{w} \\
+(\boldsymbol{w} \cdot \nabla)\left(\boldsymbol{v}_{1}-\boldsymbol{v}_{2}\right)
\end{array} \\
& \quad-\operatorname{div}\left(\left(\partial_{\xi} \nu^{*}\left(\cdot, \ell_{2}\right) \kappa\right) \nabla \mathcal{S}_{h}^{*}\left(\ell_{2}\right)\left(\left(\boldsymbol{v}_{1} \cdot \nabla\right) \boldsymbol{v}_{1}-\left(\boldsymbol{v}_{2} \cdot \nabla\right) \boldsymbol{v}_{2}\right)\right), \\
& \begin{array}{r}
\left\langle\Gamma_{\mathcal{L}}, W\right\rangle=-2 \nu^{*}\left(\cdot, \ell_{1}\right) \nabla \boldsymbol{v}_{1}: \nabla \boldsymbol{w}+2 \nu^{*}\left(\cdot, \ell_{2}\right) \nabla \boldsymbol{v}_{2}: \nabla \boldsymbol{w} \\
\quad-\partial_{\xi} \nu^{*}\left(\cdot, \ell_{1}\right) \kappa\left|\nabla \boldsymbol{v}_{1}\right|^{2}+\partial_{\xi} \nu^{*}\left(\cdot, \ell_{2}\right) \kappa\left|\nabla \boldsymbol{v}_{2}\right|^{2} .
\end{array}
\end{aligned}
$$

To conclude, we note that:

- It can be checked through technical arguments that

$$
\left\|\left\langle\Gamma_{\mathcal{S}}, W\right\rangle\right\|_{H^{-1}(\Omega)^{d}} \leq c\left\|V_{1}-V_{2}\right\|_{\mathcal{Y}}\|W\|_{\mathcal{Y}}
$$

This estimate together with (5.11) gives the Lipschitz-continuity of the first component in (5.29).

- Similar arguments also yield

$$
\left\|\left\langle\Gamma_{\mathcal{L}}, W\right\rangle\right\|_{L^{1}(\Omega)} \leq c\left\|V_{1}-V_{2}\right\|_{\mathcal{Y}}\|W\|_{\mathcal{Y}}
$$

Together with the first part of (5.25) this implies the Lipschitz-continuity of the second component in (5.29) with values in $H_{0}^{1}(\Omega)$, with Lipschitz constant smaller than $c \lambda_{h}$.

- By combining the previous estimate with the second part of (5.25), we derive that the second component in (5.29) is also Lipschitz-continuous with values in $L^{\infty}(\Omega)$, but now with Lipschitz constant smaller than $c \lambda_{h}^{2}$.

Combining these last results with (5.27) and (5.28) leads to the desired property. 
The next lemma relies on estimates (5.12), (5.14) and (5.15). We also derive the following result by combining (5.15) and (5.16), using the imbedding of $H^{s+s_{d}}(\Omega)$ into $W^{s, \infty}(\Omega)$ for a any real number $s_{d}>\frac{d}{2}$ and applying an interpolation argument: For any data $G$ in $H^{-1}(\Omega)^{d}$ such that $\mathcal{L} G$ belongs to $H^{s+s_{d}}(\Omega)$ for $1 \leq s \leq 3$,

$$
\left\|\left(\mathcal{L}-\mathcal{L}_{h}\right) G\right\|_{L^{\infty}(\Omega)} \leq c h^{s}\|\mathcal{L} G\|_{H^{s+s_{d}(\Omega)}} .
$$

Lemma 5.6. Let $\left(\boldsymbol{u}^{*}, p^{*}, k^{*}\right)$ be a solution of problem (4.4) which belongs to $H^{s+1}(\Omega)^{d} \times$ $H^{s}(\Omega) \times H^{s+s_{d}}(\Omega), 0<s \leq 2$, for a fixed real number $s_{d}>\frac{d}{2}$. When property (5.16) holds, the following estimate is satisfied

$$
\left\|\mathcal{F}_{h}\left(U^{*}\right)\right\|_{\mathcal{Y}} \leq c\left(\boldsymbol{u}^{*}, p^{*}, k^{*}\right) h^{s},
$$

where the constant $c\left(\boldsymbol{u}^{*}, p^{*}, k^{*}\right)$ only depends on the solution $\left(\boldsymbol{u}^{*}, p^{*}, k^{*}\right)$.

Proof: We have

$$
\left\|\mathcal{F}_{h}\left(U^{*}\right)\right\|_{\mathcal{Y}}=\left\|\widetilde{\mathcal{F}}\left(U^{*}\right)-\mathcal{F}_{h}\left(U^{*}\right)\right\|_{\mathcal{Y}}=\left\|\left(\mathcal{M}\left(k^{*}\right)-\mathcal{M}_{h}\left(k^{*}\right)\right) \widetilde{\mathcal{G}}\left(U^{*}\right)\right\|_{\mathcal{Y}}
$$

so that the lemma is a direct consequence of (5.12), (5.14) and (5.30) together with the regularity properties of $U^{*}=-\mathcal{M}\left(k^{*}\right) \widetilde{\mathcal{G}}\left(U^{*}\right)$.

Thanks to Lemmas 5.2, 5.5 and 5.6, we are in a position to apply the Brezzi-RappazRaviart theorem [9]. We also recall the existence of a discrete inf-sup condition between the spaces $X_{h}$ and $M_{h}$, see [20, Chap. II, Cor. 4.1]: There exists a constant $\beta>0$ independent of $h$ such that

$$
\forall q_{h} \in M_{h}, \quad \sup _{\boldsymbol{v}_{h} \in X_{h}} \frac{-\int_{\Omega}\left(\operatorname{div} \boldsymbol{v}_{h}\right)(\boldsymbol{x}) q_{h}(\boldsymbol{x}) d \boldsymbol{x}}{\left\|\boldsymbol{v}_{h}\right\|_{H^{1}(\Omega)^{d}}} \geq \beta\left\|q_{h}\right\|_{L^{2}(\Omega)} .
$$

This leads to the main result of this section.

Theorem 5.7. Let $\left(\boldsymbol{u}^{*}, p^{*}, k^{*}\right)$ be a solution of problem $(4.4)$ which belongs to $H^{s+1}(\Omega)^{d} \times$ $H^{s}(\Omega) \times H^{s+s_{d}}(\Omega), d-2<s \leq 2$, for a fixed real number $s_{d}>\frac{d}{2}$, and such that $D \mathcal{F}_{*}\left(U^{*}\right)$, with $U^{*}=\left(\boldsymbol{u}^{*}, k^{*}\right)^{\mathrm{T}}$, is an isomorphism of $\mathcal{Y}$. We moreover assume that property (5.16) holds, that the function $\nu$ belongs to $W^{2, \infty}(\mathbb{R})$ and that

$$
\lim _{h \rightarrow 0} \lambda_{h}^{2} h^{s}=0 \text {, }
$$

where $\lambda_{h}$ is defined in (5.21). Then, there exist positive numbers $\kappa$ and $h_{0}$ such that, for any $h \leq h_{0}$, problem (5.3) has a unique solution $\left(\boldsymbol{u}_{h}, p_{h}, k_{h}\right)$ such that $\left(\boldsymbol{u}_{h}, k_{h}\right)$ belongs to the ball of $\mathcal{Y}$ with centre $\left(\boldsymbol{u}^{*}, k^{*}\right)$ and radius $\kappa \lambda_{h}^{-2}$. Moreover this solution satisfies

$$
\left\|\boldsymbol{u}^{*}-\boldsymbol{u}_{h}\right\|_{H^{1}(\Omega)^{d}}+\left\|p^{*}-p_{h}\right\|_{L^{2}(\Omega)}+\left\|k^{*}-k_{h}\right\|_{H^{1}(\Omega) \cap L^{\infty}(\Omega)} \leq c\left(\boldsymbol{u}^{*}, p^{*}, k^{*}\right) h^{s},
$$

where the constant $c\left(\boldsymbol{u}^{*}, p^{*}, k^{*}\right)$ only depends on the solution $\left(\boldsymbol{u}^{*}, p^{*}, k^{*}\right)$.

Estimate (5.34) is fully optimal since it leads to a convergence order equal to 2 for very smooth solutions $\left(\boldsymbol{u}^{*}, p^{*}, k^{*}\right)$. Moreover assumption (5.33) is not at all restrictive in dimension $d=2$. But neither this assumption nor the regularity property required on the solution $\left(\boldsymbol{u}^{*}, p^{*}, k^{*}\right)$ is likely in dimension $d=3$. However the convergence of the discretization when the following condition holds

$$
\lim _{h \rightarrow 0} h_{\min }^{-1} h^{2}=0,
$$

can easily be derived from Theorem 5.7. This last condition is now much more likely and can be enforced in the adaptivity process. 


\section{A posteriori estimates.}

For each $K$ in $\mathcal{T}_{h}$, we introduce the set $\mathcal{E}_{K}$ of edges $(d=2)$ or faces $(d=3)$ of $K$ which are not contained in $\partial \Omega$. The diameters (or lengths) of $K$ and of each $e$ in $\mathcal{E}_{K}$ are denoted by $h_{K}$ and $h_{e}$, respectively. For each $e$ in $\mathcal{E}_{K},[\cdot]_{e}$ stands for the jump through $e$ in a fixed direction (it seems useless to make the sign precise). We also consider the space

$$
Z_{h}=\left\{\boldsymbol{v}_{h} \in L^{2}(\Omega)^{d} ; \forall K \in \mathcal{T}_{h},\left.\boldsymbol{v}_{h}\right|_{K} \in \mathcal{P}_{0}(K)^{d}\right\}
$$

and, assuming that the datum $\boldsymbol{f}$ belongs to $L^{2}(\Omega)^{d}$, we choose an approximation $\boldsymbol{f}_{h}$ of $\boldsymbol{f}$ in $Z_{h}$.

We again use the real number $\rho$ defined in (4.14) and we set

$$
\frac{1}{\rho}+\frac{1}{\rho^{\prime}}=1
$$

We are now in a position to define the two types of error indicators which are needed for applying the algorithm described in Section 2.

(i) An indicator linked to the modelization error

The definition of this indicator relies on Proposition 4.5. The error indicator $\eta^{m}$ is given by

$$
\eta^{m}=\left\|\operatorname{div}\left(\mu^{*}\left(\cdot, k_{h}\right) \nabla \boldsymbol{u}_{h}\right)\right\|_{W^{-1, \rho}(\Omega)^{d}}+\left\|\mu^{*}\left(\cdot, k_{h}\right)\left|\nabla \boldsymbol{u}_{h}\right|^{2}\right\|_{W^{-1, \rho}(\Omega)},
$$

where the function $\mu^{*}$ is defined in (4.16).

(ii) Indicators linked to the finite element error

These indicators are of residual type and are now standard both for the Stokes and Laplace equations, see $[41, \S 1.2]$. For each $K$ in $\mathcal{T}_{h}$, the error indicator $\eta_{K}$ is given by

$$
\eta_{K}=\eta_{K}^{\mathcal{S}}+\eta_{K}^{\mathcal{L}}
$$

with

$$
\begin{array}{r}
\eta_{K}^{\mathcal{S}}=h_{K}\left\|\boldsymbol{f}_{h}+\operatorname{div}\left(\nu^{*}\left(\cdot, k_{h}\right) \nabla \boldsymbol{u}_{h}\right)-\left(\boldsymbol{u}_{h} \cdot \nabla\right) \boldsymbol{u}_{h}-\operatorname{grad} p_{h}\right\|_{L^{\rho}(K)^{d}} \\
+\sum_{e \in \mathcal{E}_{K}} h_{e}^{\frac{1}{\rho}}\left\|\left[\nu^{*}\left(\cdot, k_{h}\right) \partial_{n} \boldsymbol{u}_{h}\right]_{e}\right\|_{L^{\rho}(e)^{d}}+\left\|\operatorname{div} \boldsymbol{u}_{h}\right\|_{L^{\rho}(K)}, \\
\eta_{K}^{\mathcal{L}}=h_{K}\left\|\nu^{*}\left(\cdot, k_{h}\right)\left|\nabla \boldsymbol{u}_{h}\right|^{2}+\alpha \Delta k_{h}\right\|_{L^{\rho}(K)}+\sum_{e \in \mathcal{E}_{K}} h_{e}^{\frac{1}{\rho}}\left\|\left[\alpha \partial_{n} k_{h}\right]_{e}\right\|_{L^{\rho}(e)} .
\end{array}
$$

It must be noted that each term which appears in the definition of the indicators $\eta_{K}$ is easy to compute once the discrete solution $\left(\boldsymbol{u}_{h}, p_{h}, k_{h}\right)$ is known.

In a first step, we intend to prove an upper bound for the error between a solution $(\boldsymbol{u}, p, k)$ of problem (3.3) and the solution $\left(\boldsymbol{u}_{h}, p_{h}, k_{h}\right)$ of problem (5.3) in a neighbourhood of it (see Proposition 4.5 and Theorem 5.7), in the norm of the space $W_{0}^{1, \rho}(\Omega)^{d} \times L^{\rho}(\Omega) \times$ $W_{0}^{1, \rho}(\Omega)$. Relying on the triangle inequality

$$
\left\|\boldsymbol{u}-\boldsymbol{u}_{h}\right\|_{W^{1, \rho}(\Omega)^{d}} \leq\left\|\boldsymbol{u}-\boldsymbol{u}^{*}\right\|_{W^{1, \rho}(\Omega)^{d}}+\left\|\boldsymbol{u}^{*}-\boldsymbol{u}_{h}\right\|_{W^{1, \rho}(\Omega)^{d}}
$$


and similar ones for the other unknowns, we proceed in two steps. We again use the space $\mathcal{X}$ defined in (4.8).

Proposition 6.1. If the function $\nu$ belongs to $W^{2, \infty}(\mathbb{R})$ and Assumption 4.4 is satisfied, let $(\boldsymbol{u}, p, k)$ be a solution of problem (3.3) such that $D \mathcal{F}(U)$ with $U=(\boldsymbol{u}, k)^{\mathrm{T}}$, is an isomorphism of $\mathcal{X}$. There exists a positive number $R$ only depending on this solution such that the following a posteriori error estimate holds

$$
\begin{aligned}
\left\|\boldsymbol{u}-\boldsymbol{u}^{*}\right\|_{W^{1, \rho}(\Omega)^{d}} & +\left\|k-k^{*}\right\|_{W^{1, \rho}(\Omega)} \\
& \leq c \eta^{m}+c(\boldsymbol{f})\left(\left\|\boldsymbol{u}^{*}-\boldsymbol{u}_{h}\right\|_{W^{1, \rho}\left(\Omega_{\ell}\right)^{d}}+\left\|k^{*}-k_{h}\right\|_{W^{1, \rho}\left(\Omega_{\ell}\right)}\right)
\end{aligned}
$$

for any solution $\left(\boldsymbol{u}^{*}, p^{*}, k^{*}\right)$ of problem (4.4) such that $\left(\boldsymbol{u}^{*}, k^{*}\right)$ belongs to the ball of $\mathcal{X}$ with centre $(\boldsymbol{u}, k)$ and radius $R$ and for a constant $c(\boldsymbol{f})$ only depending on the data $\boldsymbol{f}$.

Proof: It follows from Assumption 4.4 and Proposition 4.3 that the solution $\left(\boldsymbol{u}^{*}, p^{*}, k^{*}\right)$ satisfies, for a real number $q_{1}>\rho$,

$$
\nu_{0}\left\|\boldsymbol{u}^{*}\right\|_{W^{1, q_{1}}(\Omega)^{d}}+\alpha\left\|k^{*}\right\|_{W^{1, q_{1}}(\Omega)} \leq c\left(\|\boldsymbol{f}\|_{W^{-1, q_{1}}(\Omega)^{d}}+\|\boldsymbol{f}\|_{H^{-1}(\Omega)^{d}}^{2}\right) .
$$

We also need the inequality (which is easily derived by a duality argument)

$$
\forall \boldsymbol{w} \in L^{\rho}(\Omega)^{d}, \quad\|\operatorname{div} \boldsymbol{w}\|_{W^{-1, \rho}(\Omega)} \leq\|\boldsymbol{w}\|_{L^{\rho}(\Omega)^{d}} .
$$

Moreover, noting that all assumptions of Proposition 4.5 are satisfied and that the support of $\mu^{*}$ is contained in $\bar{\Omega}_{\ell}$, we derive from (4.17) combined with (6.8) that

$$
\begin{aligned}
& \left\|\boldsymbol{u}-\boldsymbol{u}^{*}\right\|_{W^{1, \rho}(\Omega)^{d}}+\left\|k-k^{*}\right\|_{W^{1, \rho}(\Omega)^{d}} \\
& \leq c\left(\eta^{m}+\left\|\mu\left(k^{*}\right) \nabla \boldsymbol{u}^{*}-\mu\left(k_{h}\right) \nabla \boldsymbol{u}_{h}\right\|_{L^{\rho}\left(\Omega_{\ell}\right)^{d \times d}}\right. \\
& \left.\quad+\left\|\mu^{*}\left(\cdot, k^{*}\right)\left|\nabla \boldsymbol{u}^{*}\right|^{2}-\mu^{*}\left(\cdot, k_{h}\right)\left|\nabla \boldsymbol{u}_{h}\right|^{2}\right\|_{W^{-1, \rho}(\Omega)}\right) .
\end{aligned}
$$

We then use the further triangle inequality

$$
\begin{aligned}
\left\|\mu\left(k^{*}\right) \nabla \boldsymbol{u}^{*}-\mu\left(k_{h}\right) \nabla \boldsymbol{u}_{h}\right\|_{L^{\rho}\left(\Omega_{\ell}\right)^{d \times d}} \leq \|\left(\mu\left(k^{*}\right)-\mu\left(k_{h}\right)\right) & \nabla \boldsymbol{u}^{*} \|_{L^{\rho}\left(\Omega_{\ell}\right)^{d \times d}} \\
& +\left\|\mu\left(k_{h}\right) \nabla\left(\boldsymbol{u}^{*}-\boldsymbol{u}_{h}\right)\right\|_{L^{\rho}\left(\Omega_{\ell}\right)^{d \times d}} .
\end{aligned}
$$

The second term in the right hand-side is obviously bounded by

$$
\left\|\mu\left(k_{h}\right) \nabla\left(\boldsymbol{u}^{*}-\boldsymbol{u}_{h}\right)\right\|_{L^{\rho}\left(\Omega_{\ell}\right)^{d \times d}} \leq\left(\nu_{2}-\nu_{0}\right)\left\|\boldsymbol{u}^{*}-\boldsymbol{u}_{h}\right\|_{W^{1, \rho}(\Omega)^{d}} .
$$

To evaluate the first term, we consider the four cases where

- $\nu_{1} \sqrt{\left(k^{*}\right)_{\varepsilon+}} \geq \nu_{2}-\nu_{0}$ and $\nu_{1} \sqrt{\left(k_{h}\right)_{\varepsilon+}} \geq \nu_{2}-\nu_{0}$,

- $\nu_{1} \sqrt{\left(k^{*}\right)_{\varepsilon+}}<\nu_{2}-\nu_{0}$ and $\nu_{1} \sqrt{\left(k_{h}\right)_{\varepsilon+}} \geq \nu_{2}-\nu_{0}$,

- $\nu_{1} \sqrt{\left(k^{*}\right)_{\varepsilon+}} \geq \nu_{2}-\nu_{0}$ and $\nu_{1} \sqrt{\left(k_{h}\right)_{\varepsilon+}}<\nu_{2}-\nu_{0}$,

- $\nu_{1} \sqrt{\left(k^{*}\right)_{\varepsilon+}}<\nu_{2}-\nu_{0}$ and $\nu_{1} \sqrt{\left(k_{h}\right)_{\varepsilon+}}<\nu_{2}-\nu_{0}$,

and observe that, in all of them,

$$
\left|\mu\left(k^{*}\right)-\mu\left(k_{h}\right)\right| \leq \nu_{1}\left|\sqrt{\left(k^{*}\right)_{\varepsilon}}-\sqrt{\left(k_{h}\right)_{\varepsilon}}\right| .
$$


Since $q_{1}$ is $>\rho$, setting $\frac{1}{r}=\frac{1}{\rho}-\frac{1}{q_{1}}$ and noting that $W^{1, \rho}\left(\Omega_{\ell}\right)$ is imbedded in $L^{r}\left(\Omega_{\ell}\right)$, we obtain

$$
\begin{aligned}
\left\|\left(\mu\left(k^{*}\right)-\mu\left(k_{h}\right)\right) \nabla \boldsymbol{u}^{*}\right\|_{L^{\rho}\left(\Omega_{\ell}\right)^{d \times d}} \leq \nu_{1}\left\|\sqrt{\left(k^{*}\right)_{\varepsilon}}-\sqrt{\left(k_{h}\right)_{\varepsilon}}\right\|_{L^{r}\left(\Omega_{\ell}\right)}\left\|\nabla \boldsymbol{u}^{*}\right\|_{L^{q_{1}}(\Omega)^{d \times d}} \\
\leq \frac{\nu_{1}}{2 \sqrt{\varepsilon}}\left\|k^{*}-k_{h}\right\|_{W^{1, \rho}\left(\Omega_{\ell}\right)}\left\|\boldsymbol{u}^{*}\right\|_{W^{1, q_{1}}(\Omega)^{d}}
\end{aligned}
$$

where the last estimate is obtained by multiplying and dividing by $\sqrt{\left(k^{*}\right)_{\varepsilon}}+\sqrt{\left(k_{h}\right)_{\varepsilon}}$. Combining all this with (6.7) gives

$$
\left\|\mu\left(k^{*}\right) \nabla \boldsymbol{u}^{*}-\mu\left(k_{h}\right) \nabla \boldsymbol{u}_{h}\right\|_{L^{\rho}\left(\Omega_{\ell}\right)^{d \times d}} \leq c(\boldsymbol{f})\left(\left\|\boldsymbol{u}^{*}-\boldsymbol{u}_{h}\right\|_{W^{1, \rho}\left(\Omega_{\ell}\right)^{d}}+\left\|k^{*}-k_{h}\right\|_{W^{1, \rho}\left(\Omega_{\ell}\right)}\right),
$$

where the quantity $c(\boldsymbol{f})$ only depends on $\boldsymbol{f}$. On the other hand, we use the inequality

$$
\begin{aligned}
\| \mu^{*}\left(\cdot, k^{*}\right)\left|\nabla \boldsymbol{u}^{*}\right|^{2} & -\mu^{*}\left(\cdot, k_{h}\right)\left|\nabla \boldsymbol{u}_{h}\right|^{2} \|_{W^{-1, \rho}(\Omega)} \\
\leq \|\left(\mu^{*}\left(\cdot, k^{*}\right)-\right. & \left.\mu^{*}\left(\cdot, k_{h}\right)\right)\left|\nabla \boldsymbol{u}^{*}\right|^{2} \|_{W^{-1, \rho}(\Omega)} \\
& +\left\|\mu^{*}\left(\cdot, k_{h}\right) \nabla\left(\boldsymbol{u}^{*}+\boldsymbol{u}_{h}\right) \cdot \nabla\left(\boldsymbol{u}^{*}-\boldsymbol{u}_{h}\right)\right\|_{W^{-1, \rho}(\Omega)},
\end{aligned}
$$

whence, by combining the same arguments as previously with the imbedding of $L^{r}(\Omega)$ into $W^{-1, \rho}(\Omega)$ for $\frac{1}{r}=\frac{1}{\rho}+\frac{1}{d}$ and again using $(6.7)$,

$$
\begin{aligned}
& \| \mu^{*}\left(\cdot, k^{*}\right)\left|\nabla \boldsymbol{u}^{*}\right|^{2}-\mu^{*}\left(\cdot, k_{h}\right)\left|\nabla \boldsymbol{u}_{h}\right|^{2} \|_{W^{-1, \rho}(\Omega)} \\
& \leq c(\boldsymbol{f})\left(\left\|\boldsymbol{u}^{*}-\boldsymbol{u}_{h}\right\|_{W^{1, \rho}\left(\Omega_{\ell}\right)^{d}}+\left\|k^{*}-k_{h}\right\|_{W^{1, \rho}\left(\Omega_{\ell}\right)}\right) .
\end{aligned}
$$

Combining (6.10) and (6.11) with (6.9) leads to the desired estimate.

To prove the analogous estimate for the pressure, we need the following inf-sup condition which is proven in [2, Cor. 3.2] for instance: There exists a constant $\beta_{\rho}$ such that

$$
\forall q \in L^{\rho}(\Omega), \quad \sup _{\boldsymbol{v} \in W_{0}^{1, \rho^{\prime}}(\Omega)^{d}} \frac{-\int_{\Omega}(\operatorname{div} \boldsymbol{v})(\boldsymbol{x}) q(\boldsymbol{x}) d \boldsymbol{x}}{\|\boldsymbol{v}\|_{W^{1, \rho^{\prime}}(\Omega)^{d}}} \geq \beta_{\rho}\|q\|_{L^{\rho}(\Omega)} .
$$

Note that this condition holds with $\rho$ replaced by any $r, 1<r<+\infty$. Indeed, we observe from (3.3) and (4.4) that, for any function $\boldsymbol{v}$ in $H_{0}^{1}(\Omega)^{d}$,

$$
\begin{aligned}
-\int_{\Omega}(\operatorname{div} \boldsymbol{v})(\boldsymbol{x})\left(p-p^{*}\right)(\boldsymbol{x}) d \boldsymbol{x}=-\int_{\Omega}\left(\nu(k) \nabla \boldsymbol{u}-\nu^{*}\left(k^{*}\right) \nabla \boldsymbol{u}^{*}\right): \nabla \boldsymbol{v} d \boldsymbol{x} \\
-\int_{\Omega}\left((\boldsymbol{u} \cdot \nabla) \boldsymbol{u}-\left(\boldsymbol{u}^{*} \cdot \nabla\right) \boldsymbol{u}^{*}\right) \cdot \boldsymbol{v} d \boldsymbol{x} .
\end{aligned}
$$

Thus, using the density of $H_{0}^{1}(\Omega)$ into $W_{0}^{1, \rho^{\prime}}(\Omega)$, we derive from the inf-sup condition (6.12) an estimate for $\left\|p-p^{*}\right\|_{L^{\rho}(\Omega)}$ in terms of the already evaluated quantities.

Corollary 6.2. If the assumptions of Proposition 6.1 hold and for the $R$ introduced in this proposition, the following a posteriori error estimate holds

$$
\left\|p-p^{*}\right\|_{L^{\rho}(\Omega)} \leq c \eta^{m}+c(\boldsymbol{f})\left(\left\|\boldsymbol{u}^{*}-\boldsymbol{u}_{h}\right\|_{W^{1, \rho}\left(\Omega_{\ell}\right)^{d}}+\left\|k^{*}-k_{h}\right\|_{W^{1, \rho}\left(\Omega_{\ell}\right)}\right),
$$


for any solution $\left(\boldsymbol{u}^{*}, p^{*}, k^{*}\right)$ of problem (4.4) such that $\left(\boldsymbol{u}^{*}, k^{*}\right)$ belongs to the ball of $\mathcal{X}$ with centre $(\boldsymbol{u}, k)$ and radius $R$ and for a constant $c(\boldsymbol{f})$ only depending on the data $\boldsymbol{f}$.

Proving the bound for the second part of the error still relies on the theorem of J. Pousin and J. Rappaz [38]. We first observe that the triple $\left(\boldsymbol{u}_{h}, p_{h}, k_{h}\right)$ satisfies the system of residual equations: For all $\boldsymbol{v}$ in $W_{0}^{1, \rho^{\prime}}(\Omega)^{d}$ and $\boldsymbol{v}_{h}$ in $X_{h}$,

$$
\begin{aligned}
& \int_{\Omega} \nu^{*}\left(\boldsymbol{x}, k_{h}\right) \nabla \boldsymbol{u}_{h}: \nabla \boldsymbol{v} d \boldsymbol{x}+\int_{\Omega}\left(\boldsymbol{u}_{h} \cdot \nabla\right) \boldsymbol{u}_{h} \cdot \boldsymbol{v} d \boldsymbol{x}-\int_{\Omega}(\operatorname{div} \boldsymbol{v})(\boldsymbol{x}) p_{h}(\boldsymbol{x}) d \boldsymbol{x}-\langle\boldsymbol{f}, \boldsymbol{v}\rangle \\
&=-\sum_{K \in \mathcal{T}_{h}}\left(\int_{K}\left(\boldsymbol{f}+\operatorname{div}\left(\nu^{*}\left(\cdot, k_{h}\right) \nabla \boldsymbol{u}_{h}\right)-\left(\boldsymbol{u}_{h} \cdot \nabla\right) \boldsymbol{u}_{h}-\operatorname{grad} p_{h}\right)(\boldsymbol{x}) \cdot\left(\boldsymbol{v}-\boldsymbol{v}_{h}\right)(\boldsymbol{x}) d \boldsymbol{x}\right. \\
&\left.+\frac{1}{2} \sum_{e \in \mathcal{E}_{K}} \int_{e}\left[\nu^{*}\left(\cdot, k_{h}\right) \partial_{n} \boldsymbol{u}_{h}\right]_{e}(\boldsymbol{\tau}) \cdot\left(\boldsymbol{v}-\boldsymbol{v}_{h}\right)(\boldsymbol{\tau}) d \boldsymbol{\tau}\right),
\end{aligned}
$$

and, for all $\chi$ in $W_{0}^{1, \rho^{\prime}}(\Omega)$ and $\chi_{h}$ in $Y_{h}$,

$$
\begin{array}{r}
\alpha \int_{\Omega} \operatorname{grad} k_{h} \cdot \operatorname{grad} \chi d \boldsymbol{x}-\int_{\Omega} \nu^{*}\left(\boldsymbol{x}, k_{h}\right)\left|\nabla \boldsymbol{u}_{h}\right|^{2}(\boldsymbol{x}) \chi(\boldsymbol{x}) d \boldsymbol{x} \\
=-\sum_{K \in \mathcal{T}_{h}}\left(\int_{K}\left(\nu^{*}\left(\cdot, k_{h}\right)\left|\nabla \boldsymbol{u}_{h}\right|^{2}+\alpha \Delta k_{h}\right)(\boldsymbol{x})\left(\chi-\chi_{h}\right)(\boldsymbol{x}) d \boldsymbol{x}\right. \\
\left.+\frac{1}{2} \sum_{e \in \mathcal{E}_{K}} \int_{e} \alpha\left[\partial_{n} k_{h}\right]_{e}(\boldsymbol{\tau})\left(\chi-\chi_{h}\right)(\boldsymbol{\tau}) d \boldsymbol{\tau}\right)
\end{array}
$$

However, the discrete velocity $\boldsymbol{u}_{h}$ is no longer divergence-free in the general case. So we derive from the inf-sup condition (6.12), with $\rho$ replaced by $\rho^{\prime}$, the existence of a function $\overline{\boldsymbol{u}}$ in $W_{0}^{1, \rho}(\Omega)^{d}$ such that

$$
\operatorname{div} \overline{\boldsymbol{u}}=\operatorname{div} \boldsymbol{u}_{h} \quad \text { in } \Omega \quad \text { and } \quad\|\overline{\boldsymbol{u}}\|_{W^{1, \rho}(\Omega)^{d}} \leq \beta_{\rho^{\prime}}\left\|\operatorname{div} \boldsymbol{u}_{h}\right\|_{L^{\rho}(\Omega)} .
$$

Thus, when setting $\overline{\boldsymbol{u}}_{0}=\boldsymbol{u}_{h}-\overline{\boldsymbol{u}}$, equation (6.14) can equivalently be written: For all $\boldsymbol{v}$ in $W_{0}^{1, \rho^{\prime}}(\Omega)^{d}, \boldsymbol{v}_{h}$ in $X_{h}$ and $q$ in $L_{0}^{2}(\Omega)$,

$$
\begin{aligned}
& \int_{\Omega} \nu^{*}\left(\boldsymbol{x}, k_{h}\right) \nabla \overline{\boldsymbol{u}}_{0}: \nabla \boldsymbol{v} d \boldsymbol{x}+\int_{\Omega}\left(\overline{\boldsymbol{u}}_{0} \cdot \nabla\right) \overline{\boldsymbol{u}}_{0} \cdot \boldsymbol{v} d \boldsymbol{x}-\int_{\Omega}(\operatorname{div} \boldsymbol{v})(\boldsymbol{x}) p_{h}(\boldsymbol{x}) d \boldsymbol{x}-\langle\boldsymbol{f}, \boldsymbol{v}\rangle \\
&=-\sum_{K \in \mathcal{T}_{h}}\left(\int_{K}\left(\boldsymbol{f}+\operatorname{div}\left(\nu^{*}\left(\cdot, k_{h}\right) \nabla \boldsymbol{u}_{h}\right)-\left(\boldsymbol{u}_{h} \cdot \nabla\right) \boldsymbol{u}_{h}-\operatorname{grad} p_{h}\right)(\boldsymbol{x}) \cdot\left(\boldsymbol{v}-\boldsymbol{v}_{h}\right)(\boldsymbol{x}) d \boldsymbol{x}\right.\left.+\frac{1}{2} \sum_{e \in \mathcal{E}_{K}} \int_{e}\left[\nu^{*}\left(\cdot, k_{h}\right) \partial_{n} \boldsymbol{u}_{h}\right]_{e}(\boldsymbol{\tau}) \cdot\left(\boldsymbol{v}-\boldsymbol{v}_{h}\right)(\boldsymbol{\tau}) d \boldsymbol{\tau}\right)-r(\overline{\boldsymbol{u}}, \boldsymbol{v}), \\
&-\int_{\Omega}\left(\operatorname{div} \overline{\boldsymbol{u}}_{0}\right)(\boldsymbol{x}) q(\boldsymbol{x}) d \boldsymbol{x}=0,
\end{aligned}
$$

where the quantity $r(\overline{\boldsymbol{u}}, \cdot)$ is defined by

$$
\begin{aligned}
r(\overline{\boldsymbol{u}}, \boldsymbol{v})=\int_{\Omega} \nu^{*}\left(\boldsymbol{x}, k_{h}\right) \nabla \overline{\boldsymbol{u}} & : \nabla \boldsymbol{v} d \boldsymbol{x}+\int_{\Omega}(\overline{\boldsymbol{u}} \cdot \nabla) \overline{\boldsymbol{u}} \cdot \boldsymbol{v} d \boldsymbol{x} \\
& +\int_{\Omega}(\overline{\boldsymbol{u}} \cdot \nabla) \overline{\boldsymbol{u}}_{0} \cdot \boldsymbol{v} d \boldsymbol{x}+\int_{\Omega}\left(\overline{\boldsymbol{u}}_{0} \cdot \nabla\right) \overline{\boldsymbol{u}} \cdot \boldsymbol{v} d \boldsymbol{x} .
\end{aligned}
$$


When using the notation introduced in Section 4, we write the system $(6.17)-(6.15)$ in the more compact form

$$
\bar{U}_{0}+\left(\begin{array}{ll}
\mathcal{S} & 0 \\
0 & \mathcal{L}
\end{array}\right) \mathcal{G}_{*}\left(\bar{U}_{0}\right)=\left(\begin{array}{ll}
\mathcal{S} & 0 \\
0 & \mathcal{L}
\end{array}\right) \mathcal{R}\left(\bar{U}_{0}\right)
$$

where $\bar{U}_{0}$ stands for the pair $\left(\overline{\boldsymbol{u}}_{0}, k_{h}\right)$ and the two components $\mathcal{R}_{\mathcal{S}}\left(\bar{U}_{0}\right)$ and $\mathcal{R}_{\mathcal{L}}\left(\bar{U}_{0}\right)$ of $\mathcal{R}\left(\bar{U}_{0}\right)$ are defined by duality

$$
\begin{aligned}
& \forall \boldsymbol{v} \in W_{0}^{1, \rho^{\prime}}(\Omega)^{d}, \quad\left\langle\mathcal{R}_{\mathcal{S}}\left(\bar{U}_{0}\right), \boldsymbol{v}\right\rangle \\
& \begin{array}{r}
=-\sum_{K \in \mathcal{T}_{h}}\left(\int_{K}\left(\boldsymbol{f}+\operatorname{div}\left(\nu^{*}\left(\cdot, k_{h}\right) \nabla \boldsymbol{u}_{h}\right)-\left(\boldsymbol{u}_{h} \cdot \nabla\right) \boldsymbol{u}_{h}-\operatorname{grad} p_{h}\right)(\boldsymbol{x}) \cdot\left(\boldsymbol{v}-\Pi_{h} \boldsymbol{v}\right)(\boldsymbol{x}) d \boldsymbol{x}\right. \\
\left.\quad+\frac{1}{2} \sum_{e \in \mathcal{E}_{K}} \int_{e}\left[\nu^{*}\left(\cdot, k_{h}\right) \partial_{n} \boldsymbol{u}_{h}\right]_{e}(\boldsymbol{\tau}) \cdot\left(\boldsymbol{v}-\Pi_{h} \boldsymbol{v}\right)(\boldsymbol{\tau}) d \boldsymbol{\tau}\right)-r(\overline{\boldsymbol{u}}, \boldsymbol{v}), \\
\forall \chi \in W_{0}^{1, \rho^{\prime}}(\Omega), \quad\left\langle\mathcal{R}_{\mathcal{L}}\left(\bar{U}_{0}\right), \chi\right\rangle=-\sum_{K \in \mathcal{T}_{h}}\left(\int_{K}\left(\nu\left(k_{h}\right)\left|\nabla \boldsymbol{u}_{h}\right|^{2}+\alpha \Delta k_{h}\right)(\boldsymbol{x})\left(\chi-\Pi_{h} \chi\right)(\boldsymbol{x}) d \boldsymbol{x}\right. \\
\left.\quad+\frac{1}{2} \sum_{e \in \mathcal{E}_{K}} \int_{e} \alpha\left[\partial_{h} k_{h}\right]_{e}(\boldsymbol{\tau})\left(\chi-\Pi_{h} \chi\right)(\boldsymbol{\tau}) d \boldsymbol{\tau}\right),
\end{array}
\end{aligned}
$$

where the operator $\Pi_{h}$ is defined below.

As standard in a posteriori analysis, the next result requires that $\Pi_{h}$ is a Clément type operator, see [13], more precisely an operator from $L^{1}(\Omega)$ with values in $Y_{h}$ and such that, for any function $\varphi$ in $W_{0}^{1, \rho^{\prime}}(\Omega)$, for all $K$ in $\mathcal{T}_{h}$ and $e$ in $\mathcal{E}_{K}$,

$$
\begin{aligned}
& \left\|\varphi-\Pi_{h} \varphi\right\|_{L^{\rho^{\prime}(K)}} \leq c h_{K}\|\varphi\|_{W^{1, \rho^{\prime}}\left(\Delta_{K}\right)}, \\
& \left\|\varphi-\Pi_{h} \varphi\right\|_{L^{\rho^{\prime}(e)}} \leq c h_{K}^{1-\frac{1}{\rho^{\prime}}}\|\varphi\|_{W^{1, \rho^{\prime}}\left(\Delta_{e}\right)},
\end{aligned}
$$

where $\Delta_{K}$ and $\Delta_{e}$ denote the union of the elements of $\mathcal{T}_{h}$ that share at least a vertex with $K$ and $e$, respectively. Such an operator was first constructed in [13], where the first part of estimate (6.20) is proved for $\rho=\rho^{\prime}=2$ in the two-dimensional case. A modified operator is constructed in [6, Th. IX.3.11], where the first part of estimate (6.20) is extended to the case $d=3$ and to any value of $\rho$. The second part of (6.20) is proved for this same operator in [6, Cor. IX.3.12]. We are now in a position to prove the next statement.

Proposition 6.3. Assume that there exists a constant $R_{\sharp}$ such that all solutions of problem (5.3) satisfy

$$
\left\|\operatorname{div} \boldsymbol{u}_{h}\right\|_{L^{\rho}(\Omega)} \leq R_{\sharp} .
$$

If the function $\nu$ belongs to $W^{2, \infty}(\mathbb{R})$, let $\left(\boldsymbol{u}^{*}, p^{*}, k^{*}\right)$ be a solution of problem (4.4) which belongs to $\mathcal{X}$ and such that $D \mathcal{F}_{*}\left(U^{*}\right)$ with $U^{*}=\left(\boldsymbol{u}^{*}, k^{*}\right)^{\mathrm{T}}$, is an isomorphism of $\mathcal{X}$. Then, there exists a positive number $R_{b}$ only depending on this solution such that the following a posteriori error estimate holds

$$
\left\|\boldsymbol{u}^{*}-\boldsymbol{u}_{h}\right\|_{W^{1, \rho}(\Omega)^{d}}+\left\|k^{*}-k_{h}\right\|_{W^{1, \rho}(\Omega)} \leq c\left(\sum_{K \in \mathcal{T}_{h}}\left(\eta_{K}^{\rho}+h_{K}^{\rho}\left\|\boldsymbol{f}-\boldsymbol{f}_{h}\right\|_{L^{\rho}(K)^{d}}^{\rho}\right)\right)^{\frac{1}{\rho}}
$$


for a solution $\left(\boldsymbol{u}_{h}, p_{h}, k_{h}\right)$ of problem (5.3) such that $\left(\boldsymbol{u}_{h}, k_{h}\right)$ belongs to the ball of $\mathcal{X}$ with centre $\left(\boldsymbol{u}^{*}, k^{*}\right)$ and radius $R_{b}$.

Proof: We first note from (6.16) that

$$
\|\overline{\boldsymbol{u}}\|_{W^{1, \rho}(\Omega)^{d}} \leq\left(\sum_{K \in \mathcal{T}_{h}}\left(\eta_{K}^{\mathcal{S}}\right)^{\rho}\right)^{\frac{1}{\rho}}
$$

and we use the triangle inequality

$$
\left\|\boldsymbol{u}^{*}-\boldsymbol{u}_{h}\right\|_{W^{1, \rho}(\Omega)^{d}} \leq\left\|\boldsymbol{u}^{*}-\overline{\boldsymbol{u}}_{0}\right\|_{W^{1, \rho}(\Omega)^{d}}+\|\overline{\boldsymbol{u}}\|_{W^{1, \rho}(\Omega)^{d}}
$$

Indeed, the same arguments as in Section 4 yield that all the assumptions of [41, Prop. 2.1] are satisfied by the solution $U^{*}$ of problem (4.11), so that applying this proposition leads to the following result: There exists a constant $R_{0}>0$ independent of $h$ such that any solution $\bar{U}_{0}$ of (6.19) in the ball with centre $U^{*}$ and radius $R_{0}$ satisfies

$$
\left\|U^{*}-\bar{U}_{0}\right\|_{\mathcal{X}^{*}} \leq c\left\|\left(\begin{array}{cc}
\mathcal{S} & 0 \\
0 & \mathcal{L}
\end{array}\right) \mathcal{R}\left(\bar{U}_{0}\right)\right\|_{\mathcal{X}^{*}} .
$$

The pair $\left(\boldsymbol{u}_{h}=\overline{\boldsymbol{u}}_{0}+\overline{\boldsymbol{u}}, k_{h}\right)$ belongs to the ball with centre $U^{*}$ and radius $R_{b}=R_{0}+R_{\sharp}$. On the other hand, the standard properties of the operators $\mathcal{S}$ and $\mathcal{L}$ give

$$
\begin{aligned}
\left\|\boldsymbol{u}^{*}-\overline{\boldsymbol{u}}_{0}\right\|_{W^{1, \rho}(\Omega)^{d}}+\| k^{*}- & k_{h} \|_{W^{1, \rho}(\Omega)} \\
& \leq c\left(\left\|\mathcal{R}_{\mathcal{S}}\left(\bar{U}_{0}\right)\right\|_{W^{-1, \rho}(\Omega)^{d}}+\left\|\mathcal{R}_{\mathcal{L}}\left(\bar{U}_{0}\right)\right\|_{W^{-1, \rho}(\Omega)}\right) .
\end{aligned}
$$

We now evaluate successively each term in the right-hand side.

1) By combining $(6.16),(6.21)$ and the fact that $\left\|\overline{\boldsymbol{u}}_{0}\right\|_{W^{1, \rho}(\Omega)^{d}}$ is bounded, we easily derive that

$$
|r(\bar{u}, \boldsymbol{v})| \leq c\left\|\operatorname{div} \boldsymbol{u}_{h}\right\|_{L^{\rho}(\Omega)}\|\boldsymbol{v}\|_{W^{1, \rho^{\prime}}(\Omega)^{d}} .
$$

To bound the other terms in $\mathcal{R}_{\mathcal{S}}\left(\bar{U}_{0}\right)$, we use estimates (6.20), a Hölder inequality, the fact that each element of $\mathcal{T}_{h}$ is included in a finite number of $\Delta_{K}$ and $\Delta_{e}$, only depending on the regularity parameter $\sigma$, and a further triangle inequality to replace $\boldsymbol{f}$ by $\boldsymbol{f}_{h}$. All this leads to

$$
\left|\left\langle\mathcal{R}_{\mathcal{S}}\left(\bar{U}_{0}\right), \boldsymbol{v}\right\rangle\right| \leq c\left(\sum_{K \in \mathcal{T}_{h}}\left(\left(\eta_{K}^{\mathcal{S}}\right)^{\rho}+h_{K}^{\rho}\left\|\boldsymbol{f}-\boldsymbol{f}_{h}\right\|_{L^{\rho}(K)^{d}}^{\rho}\right)\right)^{\frac{1}{\rho}}\|\boldsymbol{v}\|_{W^{1, \rho^{\prime}}(\Omega)^{d}} .
$$

2) Similarly, using (6.20) and the same arguments as previously gives

$$
\left|\left\langle\mathcal{R}_{\mathcal{L}}\left(\bar{U}_{0}\right), \chi\right\rangle\right| \leq c\left(\sum_{K \in \mathcal{T}_{h}}\left(\eta_{K}^{\mathcal{L}}\right)^{\rho}\right)^{\frac{1}{\rho}}\|\chi\|_{W^{1, \rho^{\prime}}(\Omega)}
$$

Inserting (6.25) and (6.26) into (6.24) and combining this with (6.23) yield the desired estimate.

Remark 6.4. Assumption (6.21) can easily be avoided by replacing the Stokes operator $\mathcal{S}$ by the "complete" Stokes operator which associates with any data $(\boldsymbol{F}, G)$ in $H^{-1}(\Omega)^{d} \times$ 
$L_{0}^{2}(\Omega)$, the solution $(\boldsymbol{u}, p)$ of a generalized problem (4.6) (see $[41, \S 3.5]$ for more details). However we prefer to work with the operator $\mathcal{S}$ in order that the assumptions of Proposition 6.3 are coherent with their analogues in Proposition 6.1. Anyhow assumption (6.21) is not restrictive since it results from the convergence of the discretization.

Proving the estimate on the pressure now relies on the following argument. By combining (6.14) and the first equation of problem (4.4), we obtain for all $\boldsymbol{v}$ in $W_{0}^{1, \rho^{\prime}}(\Omega)^{d}$ and $\boldsymbol{v}_{h}$ in $X_{h}$,

$$
\begin{gathered}
-\int_{\Omega}(\operatorname{div} \boldsymbol{v})(\boldsymbol{x})\left(p^{*}-p_{h}\right)(\boldsymbol{x}) d \boldsymbol{x}=-\int_{\Omega} \nu^{*}\left(\boldsymbol{x}, k^{*}\right) \nabla \boldsymbol{u}^{*}: \nabla \boldsymbol{v} d \boldsymbol{x} \\
+\int_{\Omega} \nu^{*}\left(\boldsymbol{x}, k_{h}\right) \nabla \boldsymbol{u}_{h}: \nabla \boldsymbol{v} d \boldsymbol{x}-\int_{\Omega}\left(\boldsymbol{u}^{*} \cdot \nabla\right) \boldsymbol{u}^{*} \cdot \boldsymbol{v} d \boldsymbol{x}+\int_{\Omega}\left(\boldsymbol{u}_{h} \cdot \nabla\right) \boldsymbol{u}_{h} \cdot \boldsymbol{v} d \boldsymbol{x} \\
+\sum_{K \in \mathcal{T}_{h}}\left(\int_{K}\left(\boldsymbol{f}+\operatorname{div}\left(\nu^{*}\left(\cdot, k_{h}\right) \nabla \boldsymbol{u}_{h}\right)-\left(\boldsymbol{u}_{h} \cdot \nabla\right) \boldsymbol{u}_{h}-\operatorname{grad} p_{h}\right)(\boldsymbol{x}) \cdot\left(\boldsymbol{v}-\boldsymbol{v}_{h}\right)(\boldsymbol{x}) d \boldsymbol{x}\right. \\
\left.-\frac{1}{2} \sum_{e \in \mathcal{E}_{K}} \int_{e}\left[\nu^{*}\left(\cdot, k_{h}\right) \partial_{n} \boldsymbol{u}_{h}\right]_{e}(\boldsymbol{\tau}) \cdot\left(\boldsymbol{v}-\boldsymbol{v}_{h}\right)(\boldsymbol{\tau}) d \boldsymbol{\tau}\right) .
\end{gathered}
$$

Thus, the inf-sup condition (6.12) leads to a bound for $\left\|p-p_{h}\right\|_{L^{\rho}(\Omega)}$ in terms of the $\eta_{K}$ and the quantities $\left\|\boldsymbol{u}^{*}-\boldsymbol{u}_{h}\right\|_{W^{1, \rho}(\Omega)^{d}}$ and $\left\|k^{*}-k_{h}\right\|_{W^{1, \rho}(\Omega)}$ which are evaluated in Proposition 6.3 .

Corollary 6.5. If the assumptions of Proposition 6.3 hold and for the $R_{b}$ introduced in this proposition, the following a posteriori error estimate holds

$$
\left\|p^{*}-p_{h}\right\|_{L^{\rho}(\Omega)} \leq c\left(\sum_{K \in \mathcal{T}_{h}}\left(\eta_{K}^{\rho}+h_{K}^{\rho}\left\|\boldsymbol{f}-\boldsymbol{f}_{h}\right\|_{L^{\rho}(K)^{d}}^{\rho}\right)\right)^{\frac{1}{\rho}},
$$

for a solution $\left(\boldsymbol{u}_{h}, p_{h}, k_{h}\right)$ of problem (5.3) such that $\left(\boldsymbol{u}_{h}, k_{h}\right)$ belongs to the ball of $\mathcal{X}^{*}$ with centre $\left(\boldsymbol{u}^{*}, k^{*}\right)$ and radius $R_{b}$.

We are now interested in upper bounds for the error indicators.

Proposition 6.6. If Assumption 4.4 is satisfied, the following estimate holds for the indicator $\eta^{m}$ defined in (6.3),

$$
\begin{aligned}
\eta^{m} \leq c(\boldsymbol{f})\left(\left\|\boldsymbol{u}-\boldsymbol{u}^{*}\right\|_{W^{1, \rho}(\Omega)^{d}}\right. & \left.+\left\|p-p^{*}\right\|_{L^{\rho}(\Omega)}+\left\|k-k^{*}\right\|_{W^{1, \rho}(\Omega)}\right) \\
& +c^{\prime}(\boldsymbol{f})\left(\left\|\boldsymbol{u}^{*}-\boldsymbol{u}_{h}\right\|_{W^{1, \rho}\left(\Omega_{\ell}\right)^{d}}+\left\|k^{*}-k_{h}\right\|_{W^{1, \rho}\left(\Omega_{\ell}\right)}\right),
\end{aligned}
$$

for constants $c(\boldsymbol{f})$ and $c^{\prime}(\boldsymbol{f})$ only depending on the data $\boldsymbol{f}$.

Proof: It is performed in three steps.

1) By subtracting the first line of problem (4.4) from the first line of problem (3.3), we obtain for any $\boldsymbol{v}$ in $W_{0}^{1, \rho^{\prime}}(\Omega)^{d}$ and with obvious notation for the duality pairing

$$
\begin{aligned}
\int_{\Omega}\left(\nu(k) \nabla \boldsymbol{u}-\nu\left(k^{*}\right) \nabla \boldsymbol{u}^{*}\right): & \nabla \boldsymbol{v} d \boldsymbol{x}+\int_{\Omega}\left((\boldsymbol{u} \cdot \nabla) \boldsymbol{u}-\left(\boldsymbol{u}^{*} \cdot \nabla\right) \boldsymbol{u}^{*}\right) \cdot \boldsymbol{v}(\boldsymbol{x}) d \boldsymbol{x} \\
& -\int_{\Omega}(\operatorname{div} \boldsymbol{v})(\boldsymbol{x})\left(p-p^{*}\right)(\boldsymbol{x}) d \boldsymbol{x}=\left\langle\operatorname{div}\left(\mu^{*}\left(\cdot, k^{*}\right) \nabla \boldsymbol{u}^{*}\right), \boldsymbol{v}\right\rangle .
\end{aligned}
$$


Thus, we obtain

$$
\begin{aligned}
\left\|\operatorname{div}\left(\mu^{*}\left(\cdot, k^{*}\right) \nabla \boldsymbol{u}^{*}\right)\right\|_{W^{-1, \rho}(\Omega)^{d}} \leq\left\|\nu(k) \nabla \boldsymbol{u}-\nu\left(k^{*}\right) \nabla \boldsymbol{u}^{*}\right\|_{L^{\rho}(\Omega)^{d \times d}} \\
+\left\|(\boldsymbol{u} \cdot \nabla) \boldsymbol{u}-\left(\boldsymbol{u}^{*} \cdot \nabla\right) \boldsymbol{u}^{*}\right\|_{L^{\rho}(\Omega)^{d}}+\left\|p-p^{*}\right\|_{L^{\rho}(\Omega)}
\end{aligned}
$$

It follows from standard arguments and estimate (6.7) that

$$
\begin{aligned}
\left\|\operatorname{div}\left(\mu^{*}\left(\cdot, k^{*}\right) \nabla \boldsymbol{u}^{*}\right)\right\|_{W^{-1, \rho}(\Omega)^{d}} \\
\quad \leq c(\boldsymbol{f})\left(\left\|\boldsymbol{u}-\boldsymbol{u}^{*}\right\|_{W^{1, \rho}(\Omega)^{d}}+\left\|p-p^{*}\right\|_{L^{\rho}(\Omega)}+\left\|k-k^{*}\right\|_{W^{1, \rho}(\Omega)}\right) .
\end{aligned}
$$

2) Similarly, by subtracting the third line of problem (4.3) from the third line of problem (1.2), we obtain

$$
-\alpha \Delta\left(k-k^{*}\right)=\nu(k)|\nabla \boldsymbol{u}|^{2}-\nu\left(k^{*}\right)\left|\nabla \boldsymbol{u}^{*}\right|^{2}-\mu^{*}\left(\cdot, k^{*}\right)\left|\nabla \boldsymbol{u}^{*}\right|^{2},
$$

whence

$$
\left\|\mu^{*}\left(\cdot, k^{*}\right)\left|\nabla \boldsymbol{u}^{*}\right|^{2}\right\|_{W^{-1, \rho}(\Omega)} \leq c\left(\left\|k-k^{*}\right\|_{W^{1, \rho}(\Omega)^{d}}+\left\|\nu(k)|\nabla \boldsymbol{u}|^{2}-\nu\left(k^{*}\right)\left|\nabla \boldsymbol{u}^{*}\right|^{2}\right\|_{W^{-1, \rho}(\Omega)}\right) .
$$

Owing to (6.7), this yields

$$
\left\|\mu^{*}\left(\cdot, k^{*}\right)\left|\nabla \boldsymbol{u}^{*}\right|^{2}\right\|_{W^{-1, \rho}(\Omega)} \leq c(\boldsymbol{f})\left(\left\|\boldsymbol{u}-\boldsymbol{u}^{*}\right\|_{W^{1, \rho}(\Omega)^{d}}+\left\|k-k^{*}\right\|_{W^{1, \rho}(\Omega)}\right) .
$$

3) A triangle inequality and (6.8) lead to

$$
\begin{aligned}
\eta^{m} \leq\left\|\operatorname{div}\left(\mu^{*}\left(\cdot, k^{*}\right) \nabla \boldsymbol{u}^{*}\right)\right\|_{W^{-1, \rho}(\Omega)^{d}}+\left\|\mu^{*}\left(\cdot, k^{*}\right)\left|\nabla \boldsymbol{u}^{*}\right|^{2}\right\|_{W}-1, \rho(\Omega) \\
+\left\|\mu\left(k^{*}\right) \nabla \boldsymbol{u}^{*}-\mu\left(k_{h}\right) \nabla \boldsymbol{u}_{h}\right\|_{L^{\rho}\left(\Omega_{\ell}\right) d \times d} \\
+\left\|\mu^{*}\left(\cdot, k^{*}\right)\left|\nabla \boldsymbol{u}^{*}\right|^{2}-\mu^{*}\left(\cdot, k_{h}\right)\left|\nabla \boldsymbol{u}_{h}\right|^{2}\right\|_{W^{-1, \rho}(\Omega)} .
\end{aligned}
$$

We conclude by using (6.10) and (6.11), combined with (6.30) and (6.31).

Proving the upper bounds for the indicators $\eta_{K}$ presents two difficulties:

- the quantities that appear in their definition are no longer polynomial, due to the non polynomial function $\nu^{*}$,

- the norms in their definition are no longer Hilbertian.

To handle both of them, we use some arguments presented in [41, §3.3] and [41, Lemma $3.3]$.

We bound separately the terms $\eta_{K}^{\mathcal{S}}$ and $\eta_{K}^{\mathcal{L}}$. We recall from Remark 5.1 the definition of the function $\nu_{h}$ : For each $K$ in $\mathcal{T}_{h},\left.\nu_{h}\right|_{K}$ belongs to $\mathcal{P}_{2}(K)$ and is equal to $\nu^{*}\left(\boldsymbol{a}, k_{h}(\boldsymbol{a})\right)$ at each vertex or midpoint $\boldsymbol{a}$ of an edge of $K$. According to [41, §3.3], we introduce the quantities

$$
\begin{aligned}
\varepsilon_{K}^{\mathcal{S}}=h_{K} \| \operatorname{div}\left(\left(\nu^{*}\left(\cdot, k_{h}\right)-\right.\right. & \left.\left.\nu_{h}(\cdot)\right) \nabla \boldsymbol{u}_{h}\right) \|_{L^{\rho}(K)^{d}} \\
& +\sum_{e \in \mathcal{E}_{K}} h_{e}^{\frac{1}{\rho}}\left\|\left[\left(\nu^{*}\left(\cdot, k_{h}\right)-\nu_{h}(\cdot)\right) \partial_{n} \boldsymbol{u}_{h}\right]_{e}\right\|_{L^{\rho}(e)^{d}}
\end{aligned}
$$


In a first step, we prove a bound for the quantity $\varepsilon_{K}^{\mathcal{S}}$. For each $K$ in $\mathcal{T}_{h}$, we denote by $\omega_{K}$ the union of elements of $\mathcal{T}_{h}$ that share at least an edge $(d=2)$ or a face $(d=3)$ with $K$.

Lemma 6.7. The following estimate holds for all $K$ in $\mathcal{T}_{h}$,

$$
\varepsilon_{K}^{\mathcal{S}} \leq c h_{K}\left\|\operatorname{grad} k_{h}\right\|_{L^{\infty}(K)^{d}}\left\|\nabla \boldsymbol{u}_{h}\right\|_{L^{\rho}\left(\omega_{K}\right)^{d \times d}}
$$

Proof: We bound successively the two terms in $\varepsilon_{K}^{\mathcal{S}}$.

1) We have

$$
\operatorname{div}\left(\left(\nu^{*}\left(\cdot, k_{h}\right)-\nu_{h}(\cdot)\right) \nabla \boldsymbol{u}_{h}\right)=\left(\nu^{*}\left(\cdot, k_{h}\right)-\nu_{h}(\cdot)\right) \Delta \boldsymbol{u}_{h}+\operatorname{grad}\left(\nu^{*}\left(\cdot, k_{h}\right)-\nu_{h}(\cdot)\right): \nabla \boldsymbol{u}_{h},
$$

whence

$$
\begin{aligned}
& h_{K}\left\|\operatorname{div}\left(\left(\nu^{*}\left(\cdot, k_{h}\right)-\nu_{h}(\cdot)\right) \nabla \boldsymbol{u}_{h}\right)\right\|_{L^{\rho}(K)^{d}} \\
& \leq h_{K}\left\|\left(\nu^{*}\left(\cdot, k_{h}\right)-\nu_{h}(\cdot)\right)\right\|_{L^{\infty}(K)}\left\|\Delta \boldsymbol{u}_{h}\right\|_{L^{\rho}(K)^{d}} \\
& \quad+h_{K}\left\|\operatorname{grad}\left(\nu^{*}\left(\cdot, k_{h}\right)-\nu_{h}(\cdot)\right)\right\|_{L^{\infty}(K)^{d}}\left\|\nabla \boldsymbol{u}_{h}\right\|_{L^{\rho}(K)^{d \times d}}
\end{aligned}
$$

Noting that $\nu_{h}(\cdot)$ is the Lagrange interpolate of $\nu^{*}\left(\cdot, k_{h}\right)$, therefore we derive from standard estimates [6, Lemmes IX.1.1 \& IX.1.2] and the fact that $\nu^{*}\left(\cdot, k_{h}\right)$ belongs to $W^{1, \infty}(\Omega)$

$\left\|\left(\nu^{*}\left(\cdot, k_{h}\right)-\nu_{h}(\cdot)\right)\right\|_{L^{\infty}(K)}+h_{K}\left\|\operatorname{grad}\left(\nu^{*}\left(\cdot, k_{h}\right)-\nu_{h}(\cdot)\right)\right\|_{L^{\infty}(K)^{d}} \leq c h_{K}\left\|\operatorname{grad} k_{h}\right\|_{L^{\infty}(K)^{d}}$.

Combining this with a standard inverse inequality [6, Prop. VII.4.1] to bound the term $\left\|\Delta \boldsymbol{u}_{h}\right\|_{L^{d}(K)^{d}}$, we obtain

$$
h_{K}\left\|\operatorname{div}\left(\left(\nu^{*}\left(\cdot, k_{h}\right)-\nu_{h}(\cdot)\right) \nabla \boldsymbol{u}_{h}\right)\right\|_{L^{\rho}(K)^{d}} \leq c h_{K}\left\|\operatorname{grad} k_{h}\right\|_{L^{\infty}(K)^{d}}\left\|\nabla \boldsymbol{u}_{h}\right\|_{L^{\rho}(K)^{d \times d}} .
$$

2) Similarly, we have

$$
h_{e}^{\frac{1}{\rho}}\left\|\left[\left(\nu^{*}\left(\cdot, k_{h}\right)-\nu_{h}(\cdot)\right) \partial_{n} \boldsymbol{u}_{h}\right]_{e}\right\|_{L^{\rho}(e)^{d}} \leq h_{e}^{\frac{1}{\rho}}\left\|\nu^{*}\left(\cdot, k_{h}\right)-\nu_{h}\right\|_{L^{\infty}(e)}\left\|\left[\partial_{n} \boldsymbol{u}_{h}\right]_{e}\right\|_{L^{\rho}(e)^{d}}
$$

Using the properties of the Lagrange interpolation operator on $e$ together with the fact that the trace operator from $W^{1, \infty}(K)$ into $W^{1, \infty}(e)$ has a norm bounded independently of $h_{K}$, we derive

$$
\left\|\nu^{*}\left(\cdot, k_{h}\right)-\nu_{h}\right\|_{L^{\infty}(e)} \leq c h_{e}\left\|\operatorname{grad} k_{h}\right\|_{L^{\infty}(K)^{d}} .
$$

On the other hand, combining a duality argument together with a less standard inverse inequality leads to

$$
\left\|\left[\partial_{n} \boldsymbol{u}_{h}\right]_{e}\right\|_{L^{\rho}(e)^{d}} \leq c h_{e}^{-\frac{1}{\rho}}\left\|\left[\partial_{n} \boldsymbol{u}_{h}\right]_{e}\right\|_{W^{-\frac{1}{\rho}, \rho}(e)^{d}} \leq c^{\prime} h_{e}^{-\frac{1}{\rho}}\left\|\nabla \boldsymbol{u}_{h}\right\|_{L^{\rho}\left(\omega_{K}\right)^{d \times d}}
$$

All this yields

$$
h_{e}^{\frac{1}{\rho}}\left\|\left[\left(\nu^{*}\left(\cdot, k_{h}\right)-\nu_{h}(\cdot)\right) \partial_{n} \boldsymbol{u}_{h}\right]_{e}\right\|_{L^{\rho}(e)^{d}} \leq c h_{e}\left\|\operatorname{grad} k_{h}\right\|_{L^{\infty}(K)^{d}}\left\|\nabla \boldsymbol{u}_{h}\right\|_{L^{\rho}\left(\omega_{K}\right)^{d \times d}} .
$$

The desired estimate follows from (6.34) and (6.35) by noting that $h_{e} \leq h_{K}$. 
To derive the error for the $\eta_{K}^{\mathcal{S}}$, we write the residual equation (6.14) in the modified form: For all $\boldsymbol{v}$ in $W_{0}^{1, \rho^{\prime}}(\Omega)^{d}$,

$$
\begin{gathered}
\int_{\Omega}\left(\nu^{*}\left(\boldsymbol{x}, k^{*}\right) \nabla \boldsymbol{u}^{*}-\nu^{*}\left(\boldsymbol{x}, k_{h}\right) \nabla \boldsymbol{u}_{h}\right): \nabla \boldsymbol{v} d \boldsymbol{x} \\
\quad+\int_{\Omega}\left(\left(\boldsymbol{u}^{*} \cdot \nabla\right) \boldsymbol{u}^{*}-\left(\boldsymbol{u}_{h} \cdot \nabla\right) \boldsymbol{u}_{h}\right) \cdot \boldsymbol{v} d \boldsymbol{x}-\int_{\Omega}(\operatorname{div} \boldsymbol{v})(\boldsymbol{x})\left(p^{*}-p_{h}\right)(\boldsymbol{x}) d \boldsymbol{x} \\
=\sum_{K \in \mathcal{T}_{h}}\left(\int_{K}\left(\boldsymbol{f}_{h}+\operatorname{div}\left(\nu_{h}(\cdot) \nabla \boldsymbol{u}_{h}\right)-\left(\boldsymbol{u}_{h} \cdot \nabla\right) \boldsymbol{u}_{h}-\operatorname{grad} p_{h}\right)(\boldsymbol{x}) \cdot \boldsymbol{v}(\boldsymbol{x}) d \boldsymbol{x}\right. \\
+\int_{K}\left(\boldsymbol{f}-\boldsymbol{f}_{h}\right)(\boldsymbol{x}) \cdot \boldsymbol{v}(\boldsymbol{x}) d \boldsymbol{x}+\int_{K} \operatorname{div}\left(\left(\nu^{*}\left(\cdot, k_{h}\right)-\nu_{h}(\cdot)\right)(\boldsymbol{x}) \nabla \boldsymbol{u}_{h}\right) \cdot \boldsymbol{v}(\boldsymbol{x}) d \boldsymbol{x} \\
+\frac{1}{2} \sum_{e \in \mathcal{E}_{K}}\left(\int_{e}\left[\nu_{h}(\cdot) \partial_{h} \boldsymbol{u}_{h}\right]_{e}(\boldsymbol{\tau}) \cdot \boldsymbol{v}(\boldsymbol{\tau}) d \boldsymbol{\tau}\right. \\
\left.\left.\quad+\int_{e}\left[\left(\nu^{*}\left(\cdot, k_{h}\right)-\nu_{h}(\cdot)\right) \partial_{n} \boldsymbol{u}_{h}\right]_{e}(\boldsymbol{\tau}) \cdot \boldsymbol{v}(\boldsymbol{\tau}) d \boldsymbol{\tau}\right)\right) .
\end{gathered}
$$

We also have, for all $q$ in $L_{0}^{2}(\Omega)$,

$$
\int_{\Omega}\left(\operatorname{div}\left(\boldsymbol{u}-\boldsymbol{u}_{h}\right)\right)(\boldsymbol{x}) q(\boldsymbol{x}) d \boldsymbol{x}=-\int_{\Omega}\left(\operatorname{div} \boldsymbol{u}_{h}\right)(\boldsymbol{x}) q(\boldsymbol{x}) d \boldsymbol{x} .
$$

Even if equation (6.36) is rather complex, the estimate for $\eta_{K}^{\mathcal{S}}$ is now derived from these equations by standard arguments, that we present in an abridged way.

Proposition 6.8. If Assumption 4.4 is satisfied, the following estimate holds for the indicators $\eta_{K}^{\mathcal{S}}$ defined in $(6.5), K \in \mathcal{T}_{h}$,

$$
\begin{aligned}
\eta_{K}^{\mathcal{S}} \leq c(\boldsymbol{f})\left(\left\|\boldsymbol{u}^{*}-\boldsymbol{u}_{h}\right\|_{W^{1, \rho}\left(\omega_{K}\right)^{d}}+\| p^{*}-\right. & \left.p_{h}\left\|_{L^{\rho}\left(\omega_{K}\right)}+\right\| k^{*}-k_{h} \|_{W^{1, \rho}\left(\omega_{K}\right)}\right) \\
& +c\left(h_{K}\left\|\boldsymbol{f}-\boldsymbol{f}_{h}\right\|_{L^{\rho}\left(\omega_{K}\right)^{d}}+\sum_{\kappa \subset \omega_{K}} \varepsilon_{\kappa}^{\mathcal{S}}\right),
\end{aligned}
$$

for a constant $c(\boldsymbol{f})$ only depending on the data $\boldsymbol{f}$.

Proof: We evaluate separately the three terms in $\eta_{K}^{\mathcal{S}}$.

1) Setting

$$
\boldsymbol{\varphi}_{h}=\boldsymbol{f}_{h}+\operatorname{div}\left(\nu_{h}(\cdot) \nabla \boldsymbol{u}_{h}\right)-\left(\boldsymbol{u}_{h} \cdot \nabla\right) \boldsymbol{u}_{h}-\operatorname{grad} p_{h},
$$

and using [41, Lemma 3.3], we derive that, for a finite-dimensional subspace $V_{\hat{K}}$ of $H^{1}(\hat{K})^{d}$ on the reference triangle or tetrahedron $\hat{K}$, denoting by $V_{K}$ the space of functions on $K$ obtained from $V_{\hat{K}}$ by affine transformation,

$$
\left\|\boldsymbol{\varphi}_{h}\right\|_{L^{\rho}(K)^{d}} \leq c \sup _{\boldsymbol{w} \in V_{K}} \frac{\int_{K} \boldsymbol{\varphi}_{h}(\boldsymbol{x}) \psi_{K}(\boldsymbol{x}) \cdot \boldsymbol{w}(\boldsymbol{x}) d \boldsymbol{x}}{\|\boldsymbol{w}\|_{L^{\rho^{\prime}}(K)^{d}}},
$$

where $\psi_{K}$ denotes the bubble function on $K$, equal to the product of the barycentric coordinates associated with the vertices of $K$. Thus, taking $\boldsymbol{v}$ equal to $\psi_{K} \boldsymbol{w}$ in (6.36), where $\boldsymbol{w}$ runs through $V_{K}$ and using the standard inverse inequality [6, Prop. VII.4.1]

$$
\left\|\boldsymbol{w} \psi_{K}\right\|_{W^{1, \rho^{\prime}}(K)^{d}} \leq c h_{K}^{-1}\left\|\boldsymbol{w} \psi_{K}\right\|_{L^{\rho^{\prime}(K)^{d}}} \leq c h_{K}^{-1}\|\boldsymbol{w}\|_{L^{\rho^{\prime}(K)^{d}}}
$$


we obtain the desired bound for $h_{K}\left\|\varphi_{h}\right\|_{L^{\rho}(K)^{d}}$. A further triangle inequality involving $\varepsilon_{K}^{\mathcal{S}}$ leads to the bound for

$$
h_{K}\left\|\boldsymbol{f}_{h}+\operatorname{div}\left(\nu^{*}\left(\cdot, k_{h}\right) \nabla \boldsymbol{u}_{h}\right)-\left(\boldsymbol{u}_{h} \cdot \nabla\right) \boldsymbol{u}_{h}-\operatorname{grad} p_{h}\right\|_{L^{\rho}(K)^{d}} .
$$

2) Setting $\tilde{\varphi}_{h}=\left[\nu_{h}(\cdot) \partial_{n} \boldsymbol{u}_{h}\right]_{e}$, we start from the similar formula

$$
\left\|\tilde{\boldsymbol{\varphi}}_{h}\right\|_{L^{\rho}(e)^{d}} \leq c \sup _{\boldsymbol{w} \in V_{e}} \frac{\int_{e} \tilde{\boldsymbol{\varphi}}_{h}(\boldsymbol{x}) \psi_{e}(\boldsymbol{x}) \cdot \boldsymbol{w}(\boldsymbol{x}) d \boldsymbol{x}}{\|\boldsymbol{w}\|_{L^{\rho^{\prime}}(e)^{d}}},
$$

where $V_{e}$ is contructed by affine transformation from a finite-dimensional space $V_{\hat{e}}$ on the reference edge or face $\hat{e}$, and $\psi_{e}$ is the bubble function on $e$. Next, assuming that $e$ is an edge of another element $K^{\prime}$, we set

$$
\boldsymbol{v}= \begin{cases}\mathcal{R}_{e, \kappa}\left(\psi_{e} \boldsymbol{w}\right) & \text { on } \kappa \in\left\{K, K^{\prime}\right\}, \\ 0 & \text { on } \Omega \backslash\left(K \cup K^{\prime}\right),\end{cases}
$$

where $\mathcal{R}_{e, \kappa}$ denotes a lifting operator from functions on $e$ vanishing on $\partial e$ into functions on $\kappa$ vanishing on $\partial \kappa \backslash e$, constructed by affine transformation from a fixed lifting operator from $\hat{e}$ onto $\hat{K}$. This leads to the bound for $h_{e}^{\frac{1}{\rho}}\left\|\left[\nu_{h}(\cdot) \partial_{n} \boldsymbol{u}_{h}\right]_{e}\right\|_{L^{\rho}(e)^{d}}$. A further triangle inequality involving $\varepsilon_{K}^{\mathcal{S}}$ finally gives the bound for $h_{e}^{\frac{1}{\rho}}\left\|\left[\nu^{*}\left(\cdot, k_{h}\right) \partial_{n} \boldsymbol{u}_{h}\right]_{e}\right\|_{L^{\rho}(e)^{d}}$.

3) It follows from (6.37) in an obvious way that

$$
\left\|\operatorname{div} \boldsymbol{u}_{h}\right\|_{L^{\rho}(K)} \leq c\left|\boldsymbol{u}^{*}-\boldsymbol{u}_{h}\right|_{W^{1, \rho}(K)^{d}} .
$$

This concludes the proof.

The arguments for bounding $\eta_{K}^{\mathcal{L}}$ are similar but simpler. So we skip the proofs. We next introduce the quantities

$$
\varepsilon_{K}^{\mathcal{L}}=h_{K}\left\|\left(\nu^{*}\left(\cdot, k_{h}\right)-\nu_{h}(\cdot)\right)\left|\nabla \boldsymbol{u}_{h}\right|^{2}\right\|_{L^{\rho}(K)} .
$$

Lemma 6.9. The following estimate holds for all $K$ in $\mathcal{T}_{h}$,

$$
\varepsilon_{K}^{\mathcal{L}} \leq c h_{K}^{2}\left\|\operatorname{grad} k_{h}\right\|_{L^{\infty}(K)^{d}}\left\|\nabla \boldsymbol{u}_{h}\right\|_{L^{2 \rho}(K)^{d \times d}}^{2} .
$$

We need the following modified form of the residual equation (6.15): For all $\chi$ in $W_{0}^{1, \rho^{\prime}}(\Omega)$,

$$
\begin{aligned}
& \alpha \int_{\Omega} \operatorname{grad}\left(k^{*}-k_{h}\right) \operatorname{grad} \chi d \boldsymbol{x} \\
&-\int_{\Omega}\left(\nu^{*}\left(\boldsymbol{x}, k^{*}\right)\left|\nabla \boldsymbol{u}^{*}\right|^{2}-\nu^{*}\left(\boldsymbol{x}, k_{h}\right)\left|\nabla \boldsymbol{u}_{h}\right|^{2}\right)(\boldsymbol{x}) \chi(\boldsymbol{x}) d \boldsymbol{x} \\
&=\sum_{K \in \mathcal{T}_{h}}\left(\int_{K}\left(\nu_{h}(\boldsymbol{x})\left|\nabla \boldsymbol{u}_{h}\right|^{2}(\boldsymbol{x})+\alpha \Delta k_{h}\right)(\boldsymbol{x}) \chi(\boldsymbol{x}) d \boldsymbol{x}\right. \\
&-\int_{K}\left(\nu^{*}\left(\boldsymbol{x}, k_{h}\right)-\nu_{h}(\boldsymbol{x})\right)\left|\nabla \boldsymbol{u}_{h}\right|^{2}(\boldsymbol{x}) \chi(\boldsymbol{x}) d \boldsymbol{x} \\
&\left.+\frac{1}{2} \sum_{e \in \mathcal{E}_{K}} \int_{e} \alpha\left[\partial_{h} k_{h}\right]_{e}(\boldsymbol{\tau}) \chi(\boldsymbol{\tau}) d \boldsymbol{\tau}\right) .
\end{aligned}
$$


Proposition 6.10. If Assumption 4.4 is satisfied, the following estimate holds for the indicators $\eta_{K}^{\mathcal{L}}$ defined in $(6.5), K \in \mathcal{T}_{h}$,

$$
\eta_{K}^{\mathcal{L}} \leq c(\boldsymbol{f})\left(\left\|\boldsymbol{u}^{*}-\boldsymbol{u}_{h}\right\|_{W^{1, \rho}\left(\omega_{K}\right)^{d}}+\left\|k^{*}-k_{h}\right\|_{W^{1, \rho}\left(\omega_{K}\right)}\right)+c \sum_{\kappa \subset \omega_{K}} \varepsilon_{\kappa}^{\mathcal{L}},
$$

for a constant $c(\boldsymbol{f})$ only depending on the data $\boldsymbol{f}$.

These estimates are no longer optimal according to the standard criteria. But this seems unavoidable for non polynomial coefficients, even in the linear case of coefficients only depending on the space variable $\boldsymbol{x}$, see [41, §3.2]. Moreover, if $E_{h}$ denote the full error

$$
\begin{aligned}
& E_{h}=\left\|\boldsymbol{u}-\boldsymbol{u}^{*}\right\|_{W^{1, \rho}(\Omega)^{d}}+\left\|p-p^{*}\right\|_{L^{\rho}(\Omega)}+\left\|k-k^{*}\right\|_{W^{1, \rho}(\Omega)} \\
&+\left\|\boldsymbol{u}^{*}-\boldsymbol{u}_{h}\right\|_{W^{1, \rho}(\Omega)^{d}}+\left\|p^{*}-p_{h}\right\|_{L^{\rho}(\Omega)}+\left\|k^{*}-k_{h}\right\|_{W^{1, \rho}(\Omega)},
\end{aligned}
$$

we observe from Proposition 6.1 and Corollary 6.2, Proposition 6.3 and Corollary 6.5 that it admits the upper bound

$$
E_{h} \leq c \eta^{m}+c^{\prime}\left(\sum_{K \in \mathcal{T}_{h}}\left(\eta_{K}^{\rho}+h_{K}^{\rho}\left\|\boldsymbol{f}-\boldsymbol{f}_{h}\right\|_{L^{\rho}(K)^{d}}^{\rho}\right)\right)^{\frac{1}{\rho}}
$$

which is fully optimal. On the other hand, it follows from Propositions 6.6, 6.8 and 6.10 that it admits the lower bound

$$
E_{h} \geq c \eta^{m}+c^{\prime}\left(\sum_{K \in \mathcal{T}_{h}} \eta_{K}^{\rho}\right)^{\frac{1}{\rho}}-c^{\prime \prime}\left(\sum_{K \in \mathcal{T}_{h}}\left(h_{K}^{\rho}\left\|\boldsymbol{f}-\boldsymbol{f}_{h}\right\|_{L^{\rho}(K)^{d}}^{\rho}+\left(\varepsilon_{K}^{\mathcal{S}}\right)^{\rho}+\left(\varepsilon_{K}^{\mathcal{L}}\right)^{\rho}\right)\right)^{\frac{1}{\rho}},
$$

and the lack of optimality here comes from the $\varepsilon_{K}^{\mathcal{S}}$ and $\varepsilon_{K}^{\mathcal{L}}$. However, since it follows from Lemmas 6.7 and 6.9 that a local bound for these terms can be computed explicitly, the adaptivity process can be performed in the usual way with the further requirement to be cautious for the $K$ such that $\varepsilon_{K}^{\mathcal{S}}$ and $\varepsilon_{K}^{\mathcal{L}}$ are not negligible with respect to $\eta_{K}^{\mathcal{S}}$ and $\eta_{K}^{\mathcal{L}}$.

Another difficulty comes from the fact that the $W^{-1, \rho}(\Omega)$-norm which appears in the definition of $\eta^{m}$ is not local. However, when setting

$$
\eta_{K}^{m}=\left\|\mu\left(k_{h}\right) \nabla \boldsymbol{u}_{h}\right\|_{L^{\rho}(K)^{d \times d}}+\left\|\mu\left(k_{h}\right)^{\frac{1}{2}} \nabla \boldsymbol{u}_{h}\right\|_{L^{\rho}(K)^{d \times d}}^{2},
$$

and denoting by $\mathcal{T}_{h}^{\ell}$ the set of elements of $\mathcal{T}_{h}$ which are contained in $\bar{\Omega}_{\ell}$, we observe from (6.8) that

$$
\eta^{m} \leq\left(\sum_{K \in \mathcal{T}_{h}^{\ell}}\left(\eta_{K}^{m}\right)^{\rho}\right)^{\frac{1}{\rho}}
$$

So these $\eta_{K}^{m}$ can reasonably be used in the strategy proposed in Section 2.

Remark 6.11. When replacing $\nu^{*}\left(\cdot, k_{h}\right)$ by $\nu_{h}$ in the discrete problem (5.3), as suggested in Remark 5.1, it seems more natural to define the indicators $\eta_{K}^{\mathcal{S}}$ and $\eta_{K}^{\mathcal{L}}$ by

$$
\begin{aligned}
& \eta_{K}^{\mathcal{S}}=h_{K} \| \boldsymbol{f}_{h}+\operatorname{div}\left(\nu_{h}(\cdot) \nabla\right.\left.\boldsymbol{u}_{h}\right)-\left(\boldsymbol{u}_{h} \cdot \nabla\right) \boldsymbol{u}_{h}-\operatorname{grad} p_{h} \|_{L^{\rho}(K)^{d}} \\
&+\sum_{e \in \mathcal{E}_{K}} h_{e}^{\frac{1}{\rho}}\left\|\left[\nu_{h}(\cdot) \partial_{n} \boldsymbol{u}_{h}\right]_{e}\right\|_{L^{\rho}(e)^{d}}+\left\|\operatorname{div} \boldsymbol{u}_{h}\right\|_{L^{\rho}(K)}, \\
& \eta_{K}^{\mathcal{L}}=h_{K}\left\|\nu_{h}(\cdot)\left|\nabla \boldsymbol{u}_{h}\right|^{2}+\alpha \Delta k_{h}\right\|_{L^{\rho}(K)}+\sum_{e \in \mathcal{E}_{K}} h_{e}^{\frac{1}{\rho}}\left\|\left[\alpha \partial_{n} k_{h}\right]_{e}\right\|_{L^{\rho}(e)} .
\end{aligned}
$$

All the estimates of this section remains valid in this case with only a further term, equal to $\left(\sum_{K \in \mathcal{T}_{h}}\left(\left(\varepsilon_{K}^{\mathcal{S}}\right)^{\rho}+\left(\varepsilon_{K}^{\mathcal{L}}\right)^{\rho}\right)\right)^{\frac{1}{\rho}}$, in the right-hand side of $(6.22)$ and $(6.28)$. 


\section{Numerical experiments.}

According to the adaptivity strategy proposed in Section 2, we must now solve the discrete problem (5.3) for the sequence of subdomains $\Omega_{t}^{n}$ and $\Omega_{\ell}^{n}$, provided with the triangulation $\mathcal{T}_{h}^{n}$. Since problem (5.3) is nonlinear, we also use the algorithm studied in [11] in a slightly different case (see also [42]), i.e. for a given $k_{h}^{0}$ in $Y_{h}$, we iteratively solve the uncoupled problems

Find $\left(\boldsymbol{u}_{h}^{m}, p_{h}^{m}\right)$ in $X_{h} \times M_{h}$ such that

$$
\begin{aligned}
& \forall \boldsymbol{v}_{h} \in X_{h}, \quad \int_{\Omega} \nu^{*}\left(\boldsymbol{x}, k_{h}^{m-1}\right) \nabla \boldsymbol{u}_{h}^{m}: \nabla \boldsymbol{v}_{h} d \boldsymbol{x}+\int_{\Omega}\left(\boldsymbol{u}_{h}^{m} \cdot \nabla\right) \boldsymbol{u}_{h}^{m} \cdot \boldsymbol{v}_{h}(\boldsymbol{x}) d \boldsymbol{x} \\
& -\int_{\Omega}\left(\operatorname{div} \boldsymbol{v}_{h}\right)(\boldsymbol{x}) p_{h}^{m}(\boldsymbol{x}) d \boldsymbol{x}=\left\langle\boldsymbol{f}, \boldsymbol{v}_{h}\right\rangle, \\
& \forall q_{h} \in M_{h}, \quad-\int_{\Omega}\left(\operatorname{div} \boldsymbol{u}_{h}^{m}\right)(\boldsymbol{x}) q_{h}(\boldsymbol{x}) d \boldsymbol{x}=0,
\end{aligned}
$$

Find $k_{h}^{m}$ in $Y_{h}$ such that

$$
\forall \chi_{h} \in Y_{h}, \quad \alpha \int_{\Omega} \operatorname{grad} k_{h}^{m} \cdot \operatorname{grad} \chi_{h} d \boldsymbol{x}=\int_{\Omega} \nu^{*}\left(\boldsymbol{x}, k_{h}^{m-1}\right)\left|\nabla \boldsymbol{u}_{h}^{m}\right|^{2} \chi_{h}(\boldsymbol{x}) d \boldsymbol{x} .
$$

The convergence of this algorithm is proved in [11, Th. 2] only under the assumption that the data $f$ are small enough, however it seems likely in our case. The nonlinear term $\left(\boldsymbol{u}_{h}^{m} \cdot \nabla\right) \boldsymbol{u}_{h}^{m}$ in problem (7.1) is handled via the standard characteristics method, introduced in [37].

The numerical experiments that we present are performed on the code FreeFem ++ , see [23]. They correspond to the standard backward step problem. The domain $\Omega$ is $L$-shaped, given by

$$
\left.\Omega=]-4,10[\times] 0,1[\backslash]-4,0] \times] 0, \frac{1}{2}\right] .
$$

The data $\boldsymbol{f}$ are equal to zero but the boundary conditions are now that of problem (1.1), i.e.

$$
\begin{cases}\boldsymbol{u}=\boldsymbol{g} & \text { on } \partial \Omega, \\ k=0 & \text { on } \partial \Omega,\end{cases}
$$

where the function $\boldsymbol{g}$ is zero on all edges of $\partial \Omega$ except on the vertical edges contained in the lines $x=-4$ and $x=10$, where it is given by

$$
\begin{array}{r}
\boldsymbol{g}(-4, y)=\left(16\left(y-\frac{1}{2}\right)(1-y), 0\right), \quad \frac{1}{2} \leq y \leq 1, \\
\boldsymbol{g}(10, y)=(2 y(1-y), 0), \quad 0 \leq y \leq 1 .
\end{array}
$$

The parameters $\nu_{0}, \nu_{1}, \nu_{2}, \alpha$ and $\varepsilon$ satisfy the following conditions

$$
\nu_{1}=10 \nu_{0}, \quad \nu_{2}=1, \quad \alpha=10^{-3}, \quad \varepsilon=10^{-20} .
$$


The next figures deal with the part of the flow between the lines $x=-1$ and $x=5$. Figure 2 presents, for $\nu_{0}=10^{-4}$ and from top to bottom,

- the final decomposition of $\Omega$ into $\Omega_{t}$ (in light blue) and $\Omega_{\ell}$ (in dark blue),

- the final triangulation,

- and the curves of isovalues of the stream function associated with the velocity $\boldsymbol{u}_{h}$.

Figure 3 present the same quantities for $\nu_{0}=10^{-5}$.

From these two figures, it can be observed that, when $\nu_{0}$ decreases, the size of $\Omega_{t}$ and the density of triangles in this $\Omega_{t}$ increase, which seems in good coherence with the behaviour of the flow.
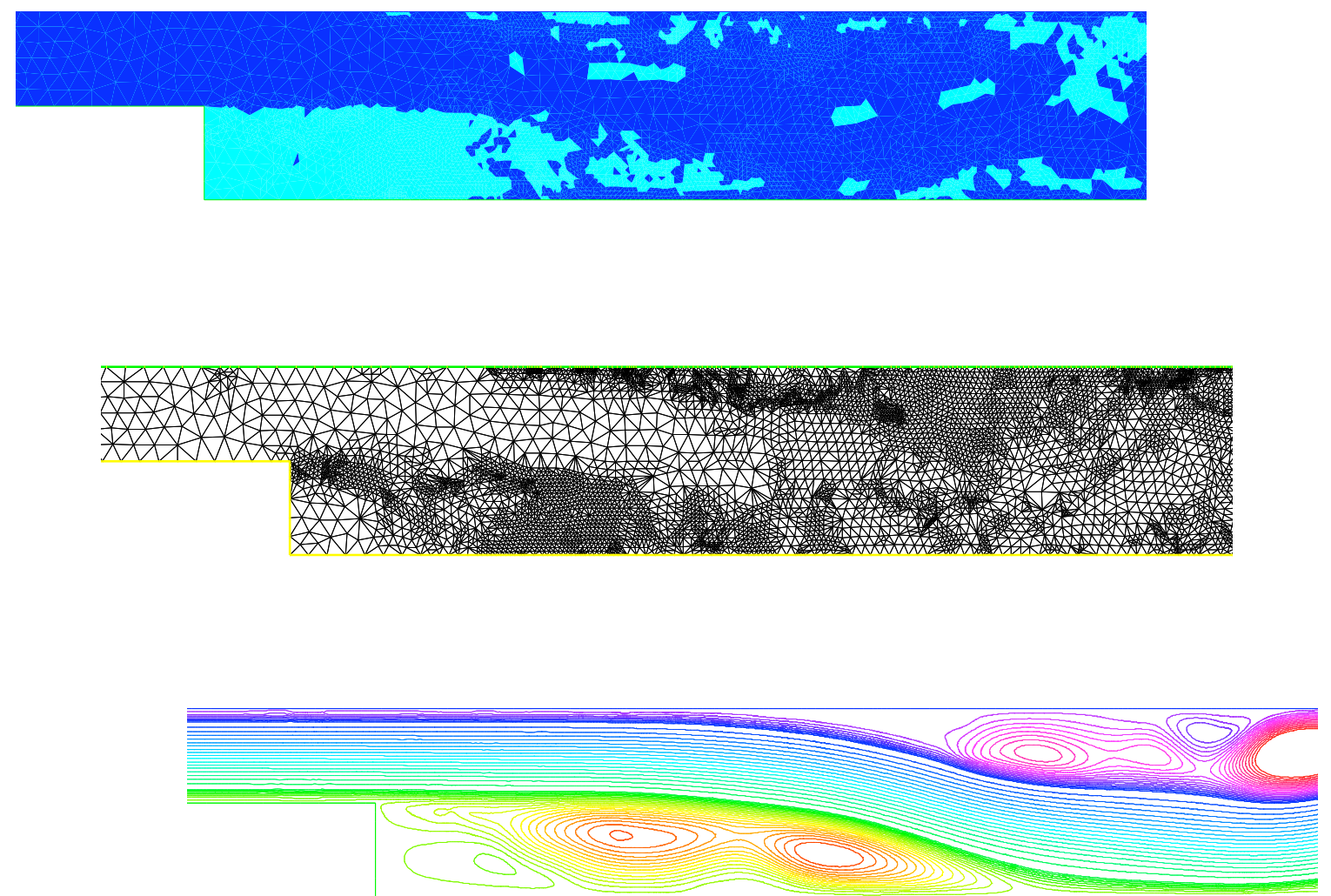

Figure 2. Decomposition, triangulation and streamlines of the flow for $\nu_{0}=10^{-4}$ 

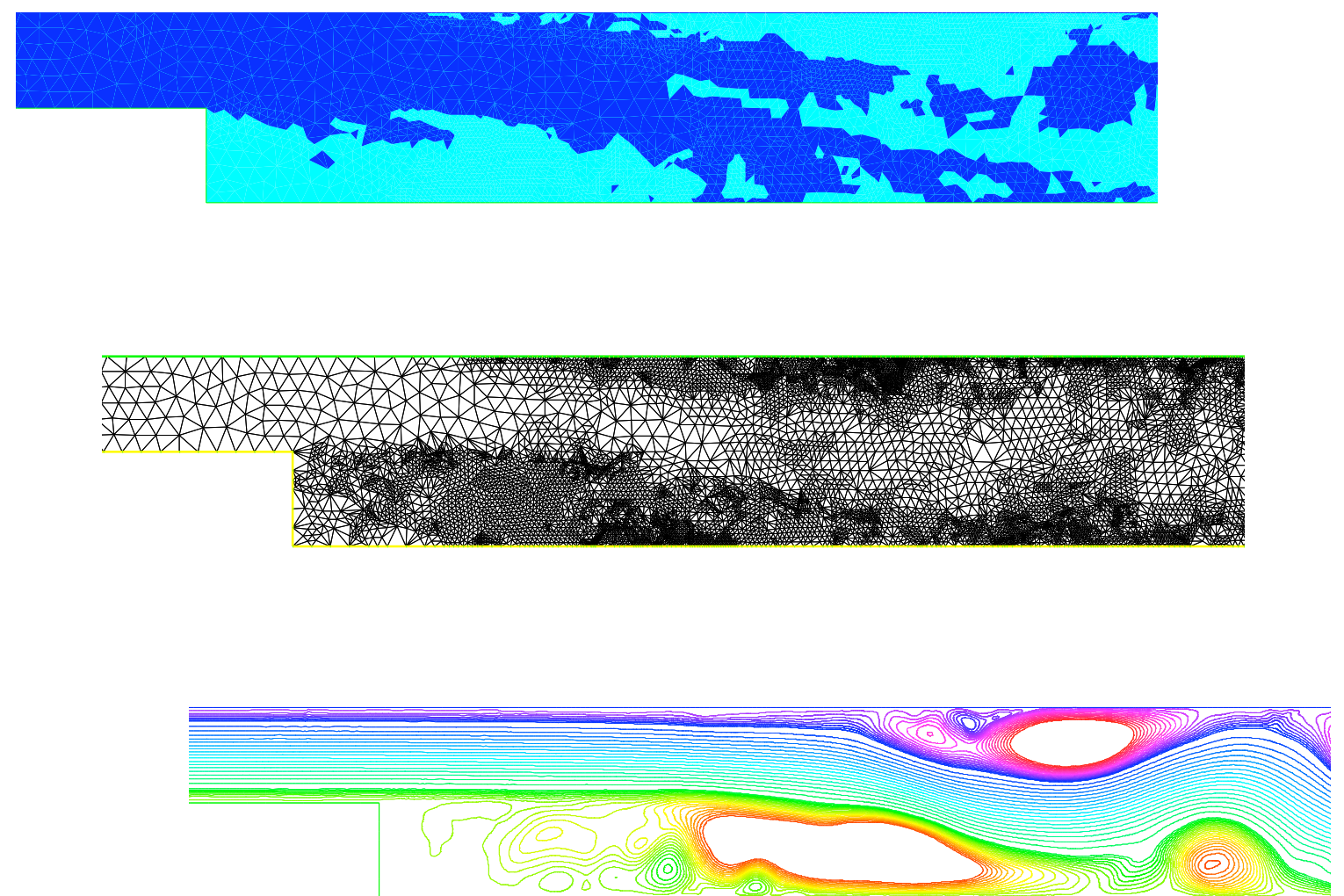

Figure 3. Decomposition, triangulation and streamlines of the flow for $\nu_{0}=10^{-5}$ 


\section{Conclusions.}

As already said in the introduction, high Reynolds number flows are not turbulent in the whole domain. Moreover, any turbulence model requires additional computational cost, in particular Reynolds Averaged Navier-Stokes (RANS) models such as the $k-\epsilon$ system and its by-products. It is also known that usually direct numerical simulations fail in real situations and therefore using the model is essential even in dimension 2 or for axisymmetric cases (see [30]). Thus, our algorithm is useful to select turbulence regions and reduce the computational cost.

Though the turbulence model that we have considered in this work is more a mathematical game than a real turbulence one, we stress that it contains the main mathematical features of a realistic RANS model. Our mathematical framework confirms that it is harder to analyze than the standard Navier-Stokes equations. Regularity assumptions are required to obtain a priori and a posteriori error estimates. Moreover, uniqueness is only proved in the two-dimenional case, with a smallness assumption on the source term which is standard for the Navier-Stokes equations. Of course, the corresponding evolution equations could have no steady state although the aim of the turbulence model is to compute statistical mean values of the real field. Nevertheless it is observed in [30] that such a model evoluates quickly to a steady state for a real physical situation; but this remains an open problem in the general case.

The numerical simulations in this present work show a good and stable numerical behavior of the selective algorithm introduced in the paper. Convergence is observed and in the case of the classical backward facing step, we get the usual structure. This makes our regularity assumptions likely. The concentration of finite elements observed in some regions however could be due to the lack of dissipation of the turbulence and near the wall to a boundary layer and the lack of wall laws. Indeed, using only one closure equation as we did is not sufficient: The mixing length and/or the turbulent dissipation must be parametrized to get a realistic scale separation. Nevertheless, the analysis and the numerical simulations that we performed make our selective algorithm promising. It should now be tested over a realistic case, though this was not the aim of this first introducing and theoretical paper.

Acknowledgement: We are very grateful toward our colleague B. Mohammadi for his help concerning the derivation of the model. This work was partially supported by the Marie Curie EIF Programme of the European Union and also by the Programa Nacional de I+D+I del Estado Español, Proyecto MTM2006-127575. 


\section{References}

[1] R.A. Adams - Sobolev Spaces, Academic Press (1975).

[2] C. Amrouche, V. Girault - Decomposition of vector spaces and application to the Stokes problem in arbitrary dimension, Czechoslovak Math. J. 44 (1994), 109-140.

[3] C. Bernardi, T. Chacón, R. Lewandowski, F. Murat - Existence d'une solution pour un modèle de deux fluides turbulents couplés, C.R. Acad. Sc. Paris 328 série I (1999), 993-998.

[4] C. Bernardi, T. Chacón Rebollo, R. Lewandowski, F. Murat - A model for two coupled turbulent fluids. Part I: analysis of the system, Collège de France Seminar XIV, D. Cioranescu \& J.-L. Lions eds., North-Holland (2002), 69-102.

[5] C. Bernardi, T. Chacón Rebollo, M. Gómez Mármol, R. Lewandowski, F. Murat - A model for two coupled turbulent fluids. Part III: Numerical approximation by finite elements, Numer. Math. 98 (2004), 33-66.

[6] C. Bernardi, Y. Maday, F. Rapetti - Discrétisations variationnelles de problèmes aux limites elliptiques, Collection "Mathématiques et Applications" 45, Springer-Verlag (2004).

[7] L. Boccardo, T. Gallouët - Nonlinear elliptic and parabolic equations involving measure data, J. Funct. Anal. 87 (1989), 149-169.

[8] M. Braack, A. Ern - A posteriori control of modeling errors and discretization errors, Multiscale Model. Simul. 1 (2003), 221-238.

[9] F. Brezzi, J. Rappaz, P.-A. Raviart - Finite dimensional approximation of nonlinear problems, Part I: Branches of nonsingular solutions, Numer. Math. 36 (1980), 1-25.

[10] F. Brossier, R. Lewandowski - Impact of the variations of the mixing length in a first order turbulent closure system, Modél. Math. Anal. Numér. 36 (2002), 345-362.

[11] T. Chacón Rebollo, S. Del Pino, D. Yakoubi - Modèle de fluide turbulent, unicité de la solution et convergence du schéma numérique, submitted.

[12] S. Clain, R. Touzani - Solution of a two-dimensional stationary induction heating problem without boundedness of the coefficients, Modél. Math. et Anal. Numér. 31 (1997), 845-870.

[13] P. Clément - Approximation by finite element functions using local regularization, R.A.I.R.O. Anal. Numér. 9 R2 (1975), 77-84.

[14] M. Dauge - Elliptic Boundary Value Problems on Corner Domains, Lecture Notes in Mathematics 1341, Springer-Verlag (1988).

[15] M. Dauge - Problèmes de Neumann et de Dirichlet sur un polyèdre dans $\mathbb{R}^{3}$ : régularité dans les espaces de Sobolev $L_{p}$, C.R. Acad. Sc. Paris 307 série I (1988), 27-32.

[16] M. Dauge - Neumann and mixed problems on curvilinear polyhedra, Integr. Equat. Oper. Th. 15 (1992), 227-261.

[17] W. Dörfler - A convergent adaptive algorithm for Poisson's equation, SIAM J. Numer. Anal. 33 (1996), 1106-1124.

[18] P.J. Frey, P.-L. George - Maillages, applications aux éléments finis, Hermès (1999).

[19] T. Gallouët, R. Herbin - Existence of a solution to a coupled elliptic system, Applied Maths Letters 2 (1994), 49-55.

[20] V. Girault, P.-A. Raviart - Finite Element Methods for Navier-Stokes Equations, Theory and Algorithms, Springer-Verlag (1986).

[21] P. Grisvard — Singularité des solutions du problème de Stokes dans un polygone, Pub. de l'Université de Nice (1978). 
[22] P. Grisvard - Elliptic Problems in Nonsmooth Domains, Pitman (1985).

[23] F. Hecht, O. Pironneau - FreeFem++, see www.freefem.org.

[24] J. Hoffman, C. Johnson - A new approach to computational turbulence modeling, Comput. Methods Appl. Mech. Engrg. 195 (2006), 2865-2880.

[25] J. Hoffman, C. Johnson - Computability and adaptivity in CFD, Chap. 7 in Encyclopedia of Computational Mechanics, Volume 3: Fluids, Wiley (2004).

[26] P. Hood, C. Taylor - A numerical solution of the Navier-Stokes equations using the finite element technique, Comp. and Fluids 1 (1973), 73-100.

[27] J. Lederer, R. Lewandowski - A RANS 3D model with unbounded eddy viscosities, Annales de l'I.H.P., Anal. Non Linéaire 24 (2007), 413-441.

[28] R. Lewandowski - Analyse mathématique et océanographie, Collection "Recherches en Mathématiques Appliquées", Masson (1997).

[29] R. Lewandowski - The mathematical analysis of the coupling of a turbulent kinetic energy equation to the Navier-Stokes equation with an eddy viscosity, Nonlinear Analysis TMA 28 (1997), 393-417.

[30] R. Lewandowski, G. Pichot - Numerical simulation of water flow around a rigid fishing net, Comput. Methods Appl. Mech. Engrg. 196 (2007), 4737-4754.

[31] J.-L. Lions, E. Magenes - Problèmes aux limites non homogènes et applications, Vol. 1, Dunod (1968).

[32] V.G. Maz'ja, B.A. Plamenevskii - The first boundary value problem for classical equations of mathematical physics in domains with piecewise smooth boundaries I, Z. Anal. Anwengundenen 2 (1983), 335-359.

[33] V.G. Maz'ja, B.A. Plamenevskii - The first boundary value problem for classical equations of mathematical physics in domains with piecewise smooth boundaries II, Z. Anal. Anwengundenen 2 (1983), 523-551.

[34] N.G. Meyers - An $L^{p}$-estimate for the gradient of solutions of second order elliptic divergence equations, Ann. Sc. Norm. Sup. Pisa 17 (1963), 189-206.

[35] B. Mohammadi, O. Pironneau - Analysis of the K-Epsilon Turbulence Model, Collection "Recherche en Mathématiques Appliquées", Masson (1994).

[36] J. Nitsche - A $L^{\infty}$-convergence of finite element approximation, Journées "Éléments Finis" de Rennes (1975).

[37] O. Pironneau - On the transport-diffusion algorithm and its applications to the Navier-Stokes equations, Numer. Math. 38 (1982), 309-332.

[38] J. Pousin, J. Rappaz - Consistency, stability, a priori and a posteriori errors for PetrovGalerkin methods applied to nonlinear problems, Numer. Math. 69 (1994), 213-231.

[39] A.H. Schatz, L.B. Wahlbin — On the quasi-optimality in $L_{\infty}$ of the $\dot{H}^{1}$-projection into finite element spaces, Math. Comput. 38 (1982), 1-22.

[40] G. Talenti - Best constant in Sobolev inequality, Ann. Math. Pura ed Appl. 110 serie IV (1976), 353-372.

[41] R. Verfürth - A Review of A Posteriori Error Estimation and Adaptive Mesh-Refinement Techniques, Wiley \& Teubner (1996).

[42] D. Yakoubi - Analyse et mise en ouvre de nouveaux algorithmes en méthodes spectrales, Ph.D. Thesis, Université Pierre et Marie Curie, Paris, in preparation. 